\title{
Erkennbarkeit angeborener Herzfehler durch eine standardisierte Untersuchung des fetalen Herzens im ersten Trimenon
}

\author{
Dissertation \\ zur Erlangung des akademischen Grades \\ doctor medicinae (Dr. med.)
}

\begin{abstract}
vorgelegt dem Rat der Medizinischen Fakultät der Friedrich-Schiller-Universität Jena
\end{abstract}

von Rosa Josephine Kähler

geboren am 07.11.1988 in Köthen 
Gutachter

1. Prof. Dr. med. Ekkehard Schleußner, Jena

2. Prof. Dr. med. Hans Proquitté, Jena

3. Prof. Dr. med. Sven Kehl, Erlangen

Tag der öffentlichen Verteidigung: 01.02.2022 


\section{Inhaltsverzeichnis}

$\underline{1}$ ABKÜRZUNGSVERZEICHNIS

$\underline{2}$ ZUSAMMENFASSUNG $\quad 8$

$\underline{3}$ EINLEITUNG 10

3.1.1 EMBRYOLOGISCHE UND PATHOPHYSIOLOGISCHE ENTWICKLUNG DES FETALEN HERZENS

3.1.2 DEFINITION UND EPIDEMIOLOGIE

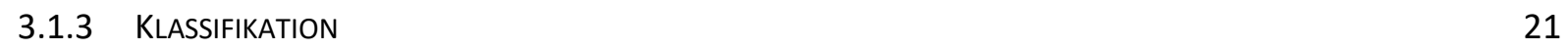

3.1.4 ÄTIOLOGIE UND RISIKEN 23

3.1.5 SYNDROMALE HERZFEHLER 26

3.1.6 PRÄVENTION

3.1.7 Perinatales Management 28

3.2 ULtRAsChall UNTERSUCHUNGEN 29

3.2.1 DIE FRÜHE FEHLBILDUNGSDIAGNOSTIK

3.2.2 DAS ERSTTRIMESTERSCREENING 30

3.2.3 Die FRÜHE FETALE HERZUNTERSUCHUNG $(11+0-13+6$ SSW) 32

3.2.4 DIE FETALE ECHOKARDIOGRAPHIE IM ZWEITEN TRIMENON

$\begin{array}{lll}3.2 .5 & \text { VORTEIL EINER FRÜHEN DIAGNOSESTELLUNG } & 37\end{array}$

$4 \quad$ ZIELE DER ARBEIT $\quad 39$

$\underline{5} \quad$ METHODIK $\quad 40$

5.1 Auswahl Patientenkollektiv $\quad 40$

5.2 KLASSIFIKATION DER DIAGNOSTIZIERTEN HERZFEHLER

5.3 Datenerhebung ZUM Patientenkollektiv

5.4. SONOGRAFISCHE UNTERSUCHUNG

5.4.1. ERSTES TRIMENON

5.4.2. ZWEITES TRIMENON 43

5.5. Prospektive Verlaufsbeobachtung 43

$\begin{array}{ll}\text { 5.6. STATISTIK } & 44\end{array}$

$\underline{6}$ ERGEBNISSE $\quad 45$

6.1 UNTERSUCHUNGSKOLLEKTIV

6.2 Prävalenz HeRzfehler $\quad 45$

6.2.1 ISOLIERTE HERZFEHLER 46

6.2.2 Chromosomale ANOMALIEN UND SYNDROMAle StÖRUNGEN 47

6.3 SENSITIVITÄT UND SPEZIFITÄT DER KARDIALEN EINSTELLUNGEN

6.4 SONOGRAFISCHE EINSTELLUNGEN IN DER FFH

6.4.1 EINZELBETRACHTUNG FETEN MIT HERZFEHLERN

6.4.2 EINZELBETRACHTUNG DER EINSTELLUNGEN

6.5 UNTERSUCHUNGSBEDINGUNGEN UND UNTERSUCHUNGSMETHODE

6.6 RISIKOFAKTOREN

6.6.1 Maternales Alter Und Maternale Adipositas 53 
6.6.2 NACKENTRANSPARENZ

6.6.3 WEITERE RISIKOFAKTOREN

6.7 SCHWANGERSCHAFTSABBRUCH UND SPONTANABORTE

6.8 FALLBESCHREIBUNG: SCHWERE UND MODERATE HERZFEHLER

6.9 FalLBESCHREIBUNG MILDE HERZFEHLER

$\mathbf{6 . 1 0}$ VERGLEICH DER KARDIALEN ULTRASCHALLBEFUNDE

6.10.1 FrüHE FETALE HERZUNTERSUCHUNG UND DIE ECHOKARDIOGRAPHIE AB DER 18. SSW BEI FETEN MIT SCHWEREN ODER MODERATEN HERZFEHLERN

6.10.2 FrüHe FetAle HeRZUNTERSUCHUNG UND DIE ECHOKARDIOGRAPHIE AB DER 18. SSW IM GESAMTEN KOLLEKTIV

6.11 Postnatales Outcome 69

6.11.1 FETEN MIT SCHWEREN ODER MODERATEN HERZFEHLERN 69

6.11.2 FETEN MIT MILDEN HERZFEHLERN

$\begin{array}{ll}\text { 6.12 Pränatal nicht diagnostizierte HerzFehler } & 70\end{array}$

7 DISKUSSION

7.1 GÜTEKRITERIEN DER KARDIALEN EINSTELLUNGEN IN DER FRÜHEN FETALEN HERZUNTERSUCHUNG 71

7.2 VERGLEICH DER KARDIALEN DIAGNOSE IN DER FRÜHEN FD UND IN DER ECHOKARDIOGRAPHIE AB DER 18. SSW BEI FETEN MIT HERZFEHLERN

7.3 KARDIALE EINSTELLUNG IN DER FRÜHEN FD - „NICHT BEURTEILBAR“ 75

7.4 VERGLEICH VON PRÄNATALER DIAGNOSE UND POSTNATALER UNTERSUCHUNG

7.5 UNTERSUCHUNGSKOLLEKTIV, RISIKOFAKTOREN UND PRÄVALENZ VON KONGENITALEN HERZFEHLERN 80

7.6 WeItere ANATOMISCHE AUFFäLLIGKEITEN UND CHROMOSOMALE STÖRUNGEN 83

$\begin{array}{lll}7.7 & \text { ABBRUCH DER SCHWANGERSCHAFT } & 85\end{array}$

$\begin{array}{lll}7.8 & \text { EINORDNUNG DER METHODE UND SICHERHEITSASPEKTE } & 87\end{array}$

8 SCHLUSSFOLGERUNG $\quad 90$

$\underline{9} \quad$ LITERATUR- UND QUELLENVERZEICHNIS $\quad 92$

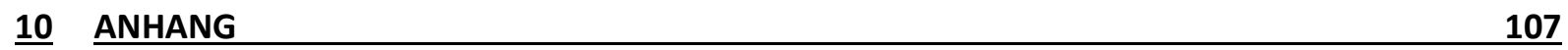

$\begin{array}{lll}10.1 & \text { AUFKLÄRUNG UND EINVERSTÄNDNISERKLÄRUNG ULTRASCHALLUNTERSUCHUNG } & 107\end{array}$

$\begin{array}{ll}10.2 & \text { PATIENTENANSCHREIBEN, EINVERSTÄNDNISERKLÄRUNG UND FRAGEBOGEN } \\ 111\end{array}$

\begin{tabular}{ll}
10.3 & AUSFÜHRUNG STATISTISCHE ERHEBUNG \\
\hline
\end{tabular}

$\begin{array}{llr}10.4 & \text { ABBILDUNGSVERZEICHNIS } & 116\end{array}$

$\begin{array}{llr}10.5 & \text { TABELLENVERZEICHNIS } & 118\end{array}$

$\begin{array}{llr}10.6 & \text { DANKSAGUNG } & 119\end{array}$

$\begin{array}{lr}10.7 & \text { LEBENSLAUF } \\ 10.8 & 120\end{array}$

$\begin{array}{llr}10.8 & \text { EHRENWÖRTLICHE ERKLÄRUNG } & 121\end{array}$ 


\section{Abkürzungsverzeichnis}

Aa

assist.

Ao

ACE

$4 \mathrm{~KB}$

AHA

anti-Ro (SSA)

anti- La (SSB)

ARSA

AS

ASD I

ASD II

ASV

AV-Block

AVSD

ß-hCG

BAS

BMI

$\mathrm{Bpm}$

CD

cfDNA

CHD

CoA

$\mathrm{CT}$

$\mathrm{CW}$

DAB

DEGUM

DGPK

DILV

DORV

DR
Arteriae

Assistierter

Aorta

Angiotensin Converting Enzyme

Vierkammerblick

American Heart Association

Sjögren-Syndrom A

Sjögren-Syndrom B

Abberant right subclavian artery $=$ Aberrante Arteria subclavia rechts

Aortenstenose

Atrium-Septumdefekt I = Ostium-primum-Defekt

Atrium-Septumdefekt II = Ostium-sekundum-Defekt

Atrioventricular septum $=$ Septum atrioventriculare

Atrioventrikulärer Block

Atrioventrikulärer Septumdefekt

free-ß-human chorionic gonadotropin

Ballonatrioseptostomie

Body mass index

Beats per minute

Color doppler

Cell free DNA = zellfreie DNA

Congenital heart defects $=$ angeborene Herzfehler

Koarktation der Aorta (s. ISTA)

Computertomographie

Continuous wave

Ductus arteriosus Botalli

Deutsche Gesellschaft für Ultraschall in der Medizin

Deutsche Gesellschaft für Pädiatrische Kardiologie

Double Inlet Left Ventricle

Double Outlet Right Ventricle

Detektionsrate 
DV

EKG

ESC

ETS

FD

FFH

FMF

GenDG

HLHS

HRST

$\mathrm{Ht}$

IAA

IAS

ISCI

ISTA

ISUOG

IUWR

IVC

IVF

LA

LSVC

LVOT

LV

MRT

MVP

NAB

NB

NBDPS

NIPT

NPW

NT

NTP

OFT

PA
Ductus venosus

Elektrokardiogramm

European Society of Cardiology

Ersttrimesterscreening

Feindiagnostik

Frühe fetale Herzuntersuchung

Fetal Medicine Foundation

Gendiagnostik-Gesetz

Hypoplastisches Linksherzsyndrom

Herzrhythmusstörung/en

heart tube $=$ Herzschlauch

Interrupted aortic arch $=$ unterbrochener Aortenbogen

Interatrial septum $=$ Vorhofseptum

Intrazytoplasmatische Spermiuminjektion

Aortenisthmusstenose

The International Society of Ultrasound in Obstetrics \& Gynecology

Intrauterine Wachstumsretardierung

Vena cava inferior

In-vitro-Fertilisation

Left atrium $=$ linker Vorhof

Persistierende linke obere Hohlvene

Left Ventricular Outflow Tract = linksventrikulärer Ausflusstrakt

Linker Ventrikel

Magnetresonanztomographie

Mitralklappenprolaps

Nasenbein

Nicht beurteilbar

National Birth Defects Prevention Study

Non-Invasive Prenatal Testing

Negativ prädiktiver Wert

Nackentransparenz

Nackentransparenz Perzentile

Outflow tract (Ausflusstrakt)

Pulmonalarterie 
PAPP-A

p.c.

PDA

PFO

PI

p.m.

PPW

pw

RA

RV

RVOT

SD

SSRI

SSW

SVC

TA

TAC

TAPVC

TGA

TI

TK

TOF

TR

$\operatorname{Tr}$

TV

USB

UVH

Vd.a.

VSD pregnancy associated plasma protein- A

$$
\text { Post conceptionem }
$$

Patent ductus arteriosus $=$ Persistierender Ductus arteriosus Botalli Persitierendes Foramen Ovale

Pulmonalklappeninsuffizienz

Post menstruationem

Positiv prädiktiver Wert

Pulsed wave

Right atrium $=$ rechter Vorhof

Right ventricle $=$ rechter Ventrikel

Right Ventricular Outflow Tract $=$ rechtsventrikulärer Ausflusstrakt

Standardabweichung

Selektive Serotonin-Wiederaufnahme-Inhibitoren

Schwangerschaftswoche

Vena cava superior

Transabdominal

Truncus arteriosus communis

Totale Lungenvenenfehlmündung

Transposition der großen Gefäße (d-TGA = dextro Transposition)

Trikuspidalinsuffizienz

Trikuspidalklappe

Fallot-Tetralogie

Trikuspidalregurgitation

Trabeculae $=$ Trabekel

Transvaginal

Untersuchungsbedingung

Univentrikuläres Herz

Verdacht auf

Ventrikelseptumdefekt 


\section{Zusammenfassung}

Angeborene Herzfehler gehören zu den häufigsten angeborenen Anomalien und sind die wichtigste Ursache der Mortalität und Morbidität im Säuglings- und Kindesalter. Die Betreuung der Schwangerschaft sowie die Planung der Entbindung und der postnatalen Versorgung des Neugeborenen erfolgt bereits unter dem Gesichtspunkt der kardialen Problematik.

Aufgrund der immer differenzierter werdenden Abbildungsqualität des Ultraschalls und der zunehmenden Erfahrung über die Bedeutung von Ultraschallbefunden in frühem Gestationsalter kann die fetale Echokardiographie bereits im ersten Trimenon durchgeführt werden. Diese ist aber an eine ausreichende Spezialisierung (DEGUM II-III) des Untersuchers mit hohem Zeitaufwand gebunden. So bleibt die frühe fetale Echokardiographie vor allem dem Hoch-Risiko-Kollektiv für Herzfehler vorbehalten.

Da jedoch Herzfehler bei der Mehrzahl aller Feten nicht in einem Risikokollektiv auftreten, ist es wünschenswert, eine Möglichkeit zu schaffen, das fetale Herz mit wenigen, in Bezug auf Sensitivität und Spezifität sicheren sonografischen Einstellungen einzuschätzen.

Ziel dieser Studie ist es daher, eine Standardisierung für die frühe kardiale Untersuchung zu etablieren, welche mehr Informationen als die alleinige Darstellung des Vierkammerblicks enthält, aber nicht so große Ressourcen bindet wie eine ausführliche fetale Echokardiographie.

Hierfür erfolgt die Untersuchung des fetalen Herzens bezüglich der Erkennbarkeit von angeborenen Herzfehlern im ersten Trimenon mittels drei sonografischer Einstellungen unter Einbeziehung des Farbdopplers: Vierkammerblick, rechte und linke Ausflussbahn.

Es wurden retrospektiv Daten von 1076 Schwangeren mit insgesamt 1183 Feten in einem Zeitraum von 01.01.2013 - 31.12.2016 aus einer DEGUM III Praxis für Pränatalmedizin analysiert. Eingeschlossen wurden Schwangere, die sowohl eine fetale Herzuntersuchung im ersten Trimenon als auch eine Echokardiographie ab der 18. SSW erhielten. Zudem erfolgte die Analyse des Studienkollektivs bezüglich bekannter Risikofaktoren für angeborene Herzfehler und mit Herzfehlern assoziierter Anomalien sowie eine detaillierte Auswertung des Verlaufs der Schwangerschaft und des postnatalen Outcomes von Feten mit schweren oder moderaten Herzfehlern.

Im Kollektiv von 1183 Feten wurden 51 Feten mit Herzfehlern gefunden (Prävalenz 4,3 \%). Davon wiesen 21 Feten (Prävalenz im Kollektiv 1,8 \%) schwere oder moderate Herzfehler auf. Bei 30 Feten wurde pränatal ein milder Herzfehler diagnostiziert. Die Studie zeigt für die 
3 im ersten Trimenon untersuchten kardialen Einstellungen bei der Einschätzung der Parameter als auffällig (,,pathologisch, kontrollbedürftig, nicht beurteilbar“") eine Sensitivität von $90,4 \%$ und eine Spezifität von 95,7 \% für die Detektion von schweren oder moderaten Herzfehlern. Der PPW ist mit $<30 \%$ bei der geringen Prävalenz von schweren und moderaten Herzfehlern (1,8 \%) relativ niedrig. Der NPW beträgt jedoch zwischen 97-99\%. Unter Einschluss milder Herzfehler reduziert sich die Sensitivität auf 39,2 \% bei einer weiterhin hohen Spezifität von 95,7 \%. Grenzt man die Auffälligkeit in der FFH bei mindestens einer der drei Einstellungen auf die Kategorie „kontrollbedürftig oder pathologisch“ ( $n=17)$ ein, so erhält man für die Frage nach moderaten oder schweren Herzfehlern eine Sensitivität von 80,9 \% und eine Spezifität von 100\%. Der PPW liegt hier bei $100 \%$ und der NPW bei 99,6 \%.

Das Kollektiv stellt sich bezüglich einer erhöhten Nackenfalte als Risikokollektiv dar. Alle anderen untersuchten Risikofaktoren zeigten keinen signifikanten Unterschied zwischen den Gruppen mit und ohne Herzfehler.

Aufgrund der in der Studie gefundenen Ergebnisse lässt sich resümieren, dass sich die drei kardialen Einstellungen als Protokoll für die Untersuchung des fetalen Herzens im ersten Trimenon eignen. Vor allem bei als unauffällig eingeschätzten Untersuchungsergebnissen liegt mit hoher Wahrscheinlichkeit kein schwerer oder moderater Herzfehler vor. Falls das Untersuchungsergebnis als „nicht beurteilbar“ eingeschätzt wird, sollte eine zeitnahe Kontrolluntersuchung ohne vorschnelle Pathologisierung erfolgen. Es kann jedoch sowohl bei auffälliger als auch bei unauffälliger kardialer Untersuchung im ersten Trimenon nicht auf eine Untersuchung zu einem späteren Zeitpunkt verzichtet werden. Dies liegt vor allem an der möglichen Entwicklung eines Herzfehlers im Laufe der Schwangerschaft, der zu einem frühen Zeitpunkt noch nicht erkennbar ist. Limitierungen zeigt die Studie zudem bei der Analyse von milden Herzfehlern. Diese können durch eine frühe fetale Herzuntersuchung mittels der drei untersuchten Einstellungen unseren Ergebnissen zufolge nicht entdeckt werden. Auch sollte bei eingeschränkten Untersuchungsbedingungen, die häufig mit einer mangelnden Einschätzung insbesondere der Ausflusstrakte einhergehen, die Begrenzung der Anwendung beachtet werden und eine erneute Herzuntersuchung im Verlauf erfolgen.

Somit ist unserer Einschätzung nach die orientierende Untersuchung des fetalen Herzens mittels der drei kardialen Einstellungen 4KB, LVOT und RVOT im ersten Trimenon vor allem zum Ausschluss schwerer und moderater Herzfehler zu empfehlen. Bei auffälligen Ergebnissen kann frühzeitig eine entsprechende Weichenstellung für den weiteren Verlauf der Schwangerschaft erfolgen. 


\section{Einleitung}

Herz- und Gefäßanomalien gehören zu den häufigsten angeborenen Fehlbildungen und bedürfen in mehr als der Hälfte der Fälle einer operativen Behandlung im Neugeborenenalter. Die vorgeburtliche Ultraschalldiagnostik trägt $\mathrm{zu}$ einer Optimierung der perinatalen, kinderkardiologischen sowie herzchirurgischen Elternberatung und der Geburts- und Therapieplanung bei. Es ist daher wünschenswert, die Diagnose eines Herzfehlers so früh wie möglich zu stellen, um die optimale Betreuung in der Zeit wichtiger Entscheidungen für Eltern und beratende Ärzte zu gewährleisten und damit das Outcome der betroffenen Kinder $\mathrm{zu}$ verbessern.

In den ersten Vorsorgeuntersuchungen für Schwangere nach Mutterschaftsrichtlinien werden maternale Risikofaktoren evaluiert und die sonografische Screeninguntersuchung zwischen $8+0$ und $11+6$ SSW durchgeführt. Hieraus ergibt sich ggf. die Indikation zur weiterführenden Untersuchung bei einem Spezialisten (DEGUM Stufe II-III). Schwangere ohne Indikation können sich auf eigenen Wunsch ebenfalls $\mathrm{zu}$ dieser weiterführenden Untersuchung vorstellen. Die dementsprechende Beratung erfolgt je nach Risikoprofil.

Inhalt dieser weiterführenden Untersuchung (oder „frühen Feindiagnostik“) ist eine detaillierte Organuntersuchung des Feten. Es kann sich, wenn von der Schwangeren gewünscht, noch eine Berechnung der fetalen Wahrscheinlichkeit für Trisomien anschließen. Diese wird auf der Basis von maternalen Parametern, der Nackenfaltendicke und maternalen Laborwerten durchgeführt.

Im Rahmen der frühen Organeinschätzung sollten Parameter in die Untersuchung einfließen, die einerseits sonografisch schnell und sicher einzustellen sind und andererseits eine hohe Sensitivität sowie Spezifität bezüglich fetaler Fehlbildungen vorweisen.

So hat speziell die Einschätzung des fetalen Herzens, dessen Fehlbildung die häufigste angeborene Anomalie darstellt, eine zentrale Rolle in der frühen fetalen Sonografie.

\subsection{Kongenitale Herzfehler}

\subsubsection{Embryologische und pathophysiologische Entwicklung des fetalen Herzens}

$\mathrm{Ab}$ der 3. SSW p.c. beginnt die Entwicklung des Herz- und Gefäßsystems. Diese ist notwendig, da der Embryo seinen Ernährungsbedarf nicht mehr durch Diffusion decken kann (Jan Langman 1989). 
Die embryonale Entwicklung des Herzens vollzieht sich dabei in drei Hauptphasen (Knöpfle 1997) s. Abb. 1:

Zunächst entwickelt sich aus der kardiogenen Platte der Herzschlauch s. Abb. 1a. In der zweiten Phase krümmt sich dieser in einem weitestgehend autonomen Prozess zur Herzschleife s. Abb. 1b, welche am Tag 28 p.c. abgeschlossen ist. Durch die Krümmungen sind ein zunächst paarig angelegtes einheitliches Atrium sowie der embryonale Ventrikel entstanden. Aus der Herzschleife entsteht dann im dritten Abschnitt durch Furchung und Septierung das vierkammerige Herz s. Abb. 1c und 1d. Hier teilt sich am Ende der 4. SSW p.c. der Vorhof mit Bildung zweier Septen und durch Apoptose induzierte Foramina. Am Ende bleibt bis zur Geburt das Foramen ovale zwischen den Vorhöfen offen, welches sich postnatal durch Druckerhöhung im linken Vorhof mit Beginn der Atmung verschließt. Im Ventrikel beginnt die Septierung ebenfalls am Ende der 4. SSW. Das Septum interventrikulare besteht am Ende aus einem muskulösen und einem membranösen Teil. Die parallel zur Entwicklung des Ventrikelseptums entstehende Trennung der ventrikulären Ausflussbahnen als RVOT in den Truncus pulmonalis und LVOT in die Aorta erfolgt durch das Septum Aorticopulmonale aus einer spiralförmigen Drehung der Trunkuswülste (Jan Langman 1989). Das fetale Herz ist dann bereits ab der 10. SSW p.c. strukturell ausgebildet (Apitz 1998) und somit sonografisch beurteilbar.

(a)

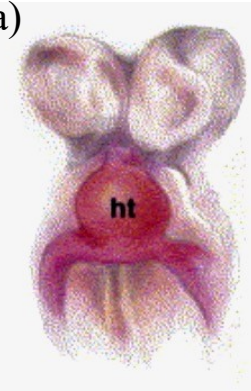

(b)

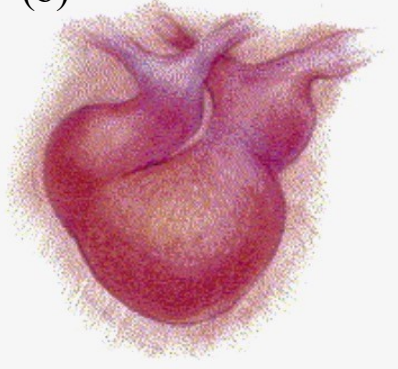

(c)

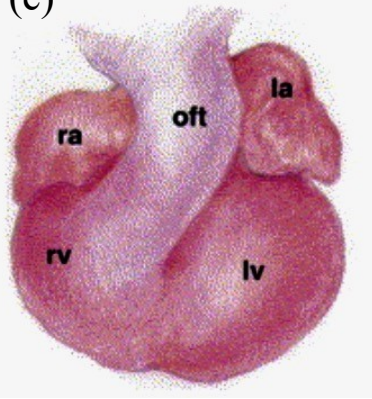

(d)

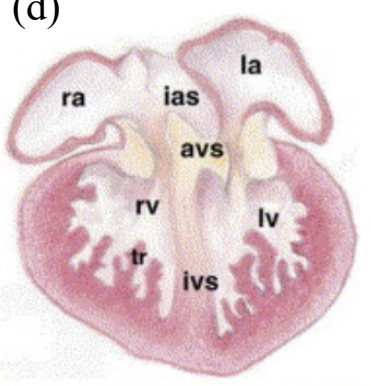

Abb. 1: Embryonale Entwicklung des Herzens (Fiona A. Stennard 2005)

$\mathrm{Ht}=$ heart tube (Herzschlauch), ra = right atrium (rechter Vorhof), la = left atrium (linker Vorhof), oft = outflow tract (Ausflusstrakt), $\mathrm{rv}=$ right ventricle (rechter Ventrikel), ias = interatrial septum (Vorhofseptum), avs $=$ atrioventricular septum (Septum atrioventriculare), ivs $=$ interventricular septum (Ventrikelseptum), $\operatorname{tr}=$ trabeculae (Trabekel) 


\section{$\underline{\text { Pathologien }}$}

Die Entstehung von Herzfehlern ist noch nicht abschließend erforscht, jedoch lassen sich einige Anomalien den jeweiligen Embryonalphasen zuordnen.

Die Lateralitätskaskade, in der zwischen Tag 16-18 p.c. Teile des rechten Ausflusstraktes zum linken Ausflusstrakt werden und umgekehrt, wird durch das Schlüsselmolekül Serotonin induziert und kann z.B. durch Psychopharmaka gestört werden. Störungen in diesem Bereich, führen u.a. zu Dextrokardie, VSD, DORV und auch zur Transposition der großen Gefäße.

Auch bei der Septierung der Vorhöfe können u.a. durch eine übermäßige Resorption des Septum primum oder eine nicht suffiziente Entwicklung des Septum secundum die häufigen ASD entstehen. Schließt sich das Formen ovale vorzeitig, kann eine massive Hypertrophie des rechten Vorhofs mit konsekutiver Atrophie des linken Herzens die Folge sein.

Störungen in der Entwicklung des Septum interventrikulare führen als VSD zu den häufigsten angeborenen Herzfehlern. 80 \% davon sind im muskulären Teil lokalisiert und können sich mit Wachstum des Kindes verschließen. Defekte im Pars membranacea sind schwerwiegender und gehen häufig mit Störung der Konus-Truncus Region wie bei der Fallot-Tetralogie einher. Defekte bei der Trennung der Ausflussbahnen sind unter anderem die Transposition der großen Arterien oder die Pulmonalstenose (Pschyrembel Redaktion 2019).

\section{Pränataler- und postnataler Kreislauf}

Pränatal wird die Sauerstoffversorgung nicht wie im adulten Kreislauf durch die Verbindung von Lungen- und Körperkreislauf gewährleistet, sondern erfolgt über die Zufuhr sauerstoffreichen Blutes aus der Plazenta über die Nabelvene und den Ductus venosus zur Vena cava inferior in den rechten Ventrikel s. Abb. 2 (Sitzmann 2002).

Der Bluthauptstrom gelangt durch das Foramen ovale in den linken Vorhof und damit in den Körperkreislauf. Ein kleiner Teil fließt durch den rechten Ventrikel in den Truncus pulmonalis und die Lungenarterien zur Lunge bzw. durch den Ductus arteriosus wieder in die Aorta descendens. Nach der Körperpassage gelangt das sauerstoffarme Blut über die beiden Nabelarterien zurück zur Plazenta.

Die physiologischen Shunts, die den Körper- und Lungenkreislauf im Feten hierbei verbinden, sind der Ductus venosus zwischen Nabelvene und Sinus venosus, das Foramen 
ovale zwischen rechtem und linken Vorhof sowie der DAB (Ductus arteriosus Botalli) zwischen Truncus pulmonalis und der Aorta.

Postnatal verschließt sich der DAB durch Muskelkontraktion, vermittelt durch das bei Belüftung der Lungen entstandene Bradykinin und durch einen Abfall der Prostaglandinkonzentration innerhalb der ersten 10-15 Lebensstunden. Daraufhin erhöht sich das Blutvolumen im Lungenkreislauf und führt $\mathrm{zu}$ einem pränatal nicht vorhandenen Druckunterschied in den Vorhöfen. Konsekutiv verschließt sich das Foramen ovale (Sitzmann 2002). Feten mit zyanotischen Herzfehlern kommen ab diesem Moment in eine Notsituation, da die physiologischen pränatalen Shunts nicht mehr offen sind.

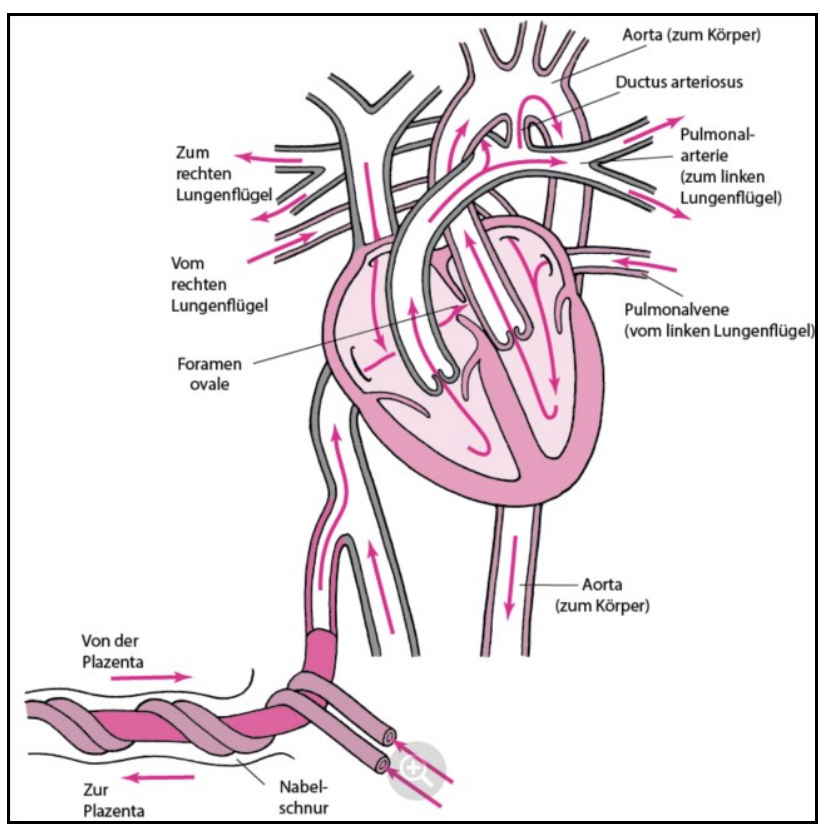

Abb. 2: physiologische Blutzirkulation bei einem Fötus (MSD Manuals: Jeanne Marie Baffa 2018)

Zyanotische Herzfehler entstehen durch einen Rechts-Links-Shunt, bei dem das sauerstoffarme Blut direkt ohne Lungenpassage in den Körperkreislauf gelangt. Dazu zählen u.a. Fallot-Tetralogie, Trikuspidal - und Pulmonalatresie, persistierender Truncus arteriosus (TAC), Transposition der großen Gefäße, Ebstein-Anomalie sowie das hypoplastische Linksherzsyndrom (HLHS).

Jedoch können auch zyanotische Herzfehler zunächst unauffällig bleiben und azyanotische Herzfehler auch später durch eine Zyanose auffällig werden (Apitz 1998).

Ductusabhängige Herzfehler dekompensieren bei Verschluss des Ductus arteriosus. Dazu zählen HLHS, kritische Aortenstenose, Aortenisthmusstenose sowie zyanotische Läsionen wie eine schwere Fallot-Tetralogie und Pulmonalatresie. 
Bei diesen Herzfehlern ist die Persistenz des Ductus arteriosus durch eine Prostaglandininfusion bis zur operativen Korrektur entscheidend für die Erstversorgung der betroffenen Kinder.

Die pränatale Diagnose ist Voraussetzung für die Detektion derjenigen fetalen Herzfehler, bei denen postnatal eine medikamentös induzierte Ductus Botalli Persistenz indiziert ist.

Im Folgenden folgt eine Erläuterung zu Herzfehlern, die Gegenstand dieser Arbeit sind.

\section{Univentrikuläres Herz:}

Zum univentrikulären Herzen zählt man eine Gruppe von Herzfehlern, bei denen keine zwei Ventrikel mit Einlassteil und Pumpteil vorhanden sind (Double Inlet Left Ventricle, Trikuspidalatresie, Hypoplastisches Linksherzsyndrom (DGPK: A. Hager et al. 2011).

\section{Double inlet left Ventricle (DILV)}

Zwei normal entwickelte Vorhöfe drainieren über zwei separate AV-Klappen in einen gemeinsamen Ventrikel. Meist existiert ein kleiner unterentwickelter rechter Ventrikel, der über einen VSD mit dem linken Ventrikel verbunden ist s. Abb. 3 (Abuhamad und Chaoui 2010).

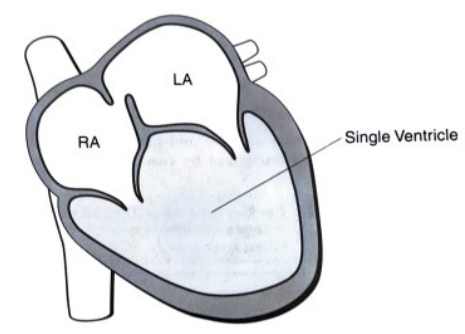

Abb. 3: Double inlet ventricle (Abuhamad und Chaoui 2010)

$\mathrm{RA}=$ rechtes Atrium; $\mathrm{LA}=$ linkes Atrium

\section{Hypoplastisches Linksherzsyndrom (HLHS)}

Unter dem HLHS wird eine Gruppe komplexer kardialer Malformationen zusammengefasst, die durch eine signifikante Unterentwicklung des linken Ventrikels und des linken Ausflusstraktes gekennzeichnet sind. Diese sind vergesellschaftet mit kritischen Stenosen bzw. Atresien der Mitral- und/oder Aortenklappe sowie einer Hypoplasie der Aorta ascendens und des Aortenbogens s. Abb. 4. Mit postnatal beginnendem Ductusverschluss sowie Abfall des Lungengefäßwiderstandes entwickelt sich ein kardiogener Schock (DGPK: N. A. Haas et al. 2013). 


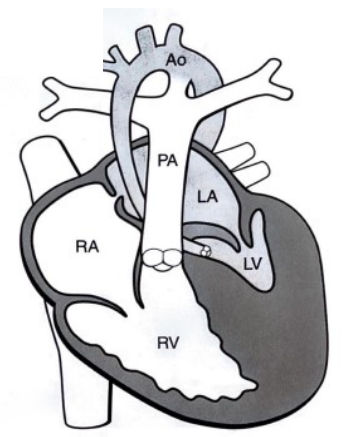

Abb. 4: HLHS: mit hypoplastischem, hypokinetischem LV, dysplastischer Mitralklappe, Aortenklappenatresie und hypoplastischem Aortenbogen (Abuhamad und Chaoui 2010)

$\mathrm{RA}=$ rechtes Atrium; RV = rechter Ventrikel; LA = linkes Atrium; LV = linker Ventrikel; PA = Pulmonalarterie; Ao $=$ Aorta

\section{Double outlet right ventricle (DORV)}

Beim ,Double Outlet Right Ventricle' (DORV) besitzen beide großen Arterien eine morphologische Konnektion zum rechten Ventrikel. Dabei besteht jedoch keine einheitliche kongenitale kardiale Malformation, sondern die Konstellation einer Malposition der großen Arterien, bei der Pulmonalarterie und Aorta ausschließlich (100\% + $100 \%)$ oder überwiegend, d.h. eine Arterie komplett $(100 \%)$ und die andere mit $>50 \%$ des Klappendurchmessers, aus dem morphologisch rechten Ventrikel entspringen. Dabei kann die Aorta vor oder hinter, rechts oder links der Pulmonalarterie liegen s. Abb. 5. Die interventrikuläre Kommunikation zwischen beiden Kammern wird überwiegend als VSD bezeichnet, obwohl sie in einigen Fällen nicht dem Kammerscheidewanddefekt entspricht (Bertram et al. 2013)

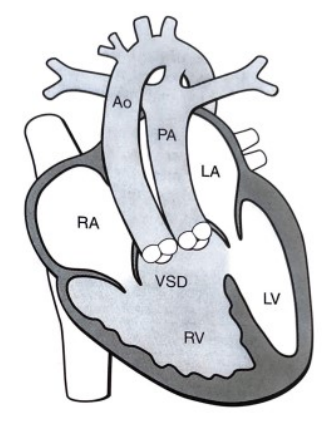

Abb. 5: Beispielvariante DORV, bei dem die Aorta rechts vor der Pulmonalarterie liegt (Abuhamad und Chaoui 2010)

$\mathrm{RA}=$ rechtes Atrium; RV = rechter Ventrikel; $\mathrm{LA}=$ linkes Atrium; $\mathrm{LV}=$ linker Ventrikel; $\mathrm{PA}=$ Pulmonalarterie; Ao = Aorta; VSD = Ventrikelseptumdefekt 


\section{Transposition der großen Gefäße (TGA)}

Die Transposition der großen Arterien ist dadurch definiert, dass die Aorta aus dem morphologisch rechten und die Pulmonalarterie aus dem morphologisch linken Ventrikel entspringen. Es besteht also eine ventrikuloarterielle Diskordanz bei atrioventrikulärer Konkordanz s. Abb. 6. Bei der anatomisch häufigsten Form nimmt die Aorta eine rechtsanteriore Malposition in Kontinuität mit dem rechtsventrikulären Ausflusstrakt ein (dTransposition, d-TGA).

Durch die Parallelschaltung von Körper- und Lungenkreislauf wird nicht-oxygeniertes systemvenöses Blut vom rechten Ventrikel in die Aorta gepumpt und das oxygenierte pulmonalvenöse Blut vom linken Ventrikel in die Pulmonalarterie. Der Begriff „einfache Transposition“ oder „d-TGA simplex“ (ca. 75\% der Fälle) wird verwendet, wenn keine weiteren Fehlbildungen vorhanden sind (Rickers et al. 2013).

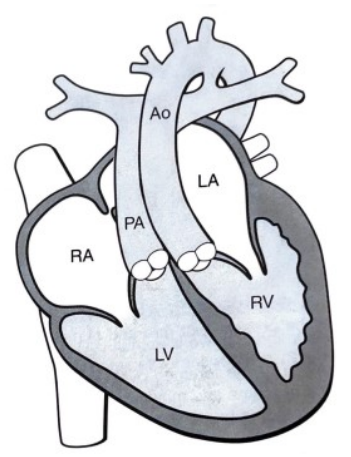

Abb. 6: Beispiel TGA mit atrioventrikulärer Diskonkordanz, RA verbunden durch Mitralklappe zu LV, LA verbunden durch Trikuspidalklappe zu RV, Aorta entspringt RV, Pulmonalarterie entspringt LV, parallelverlaufende große Gefäße, Ao liegt vor PA (Abuhamad und Chaoui 2010) $\mathrm{RA}=$ rechtes Atrium; RV = rechter Ventrikel; $\mathrm{LA}=$ linkes Atrium; $\mathrm{LV}=$ linker Ventrikel; $\mathrm{PA}=$ Pulmonalarterie; Ao $=$ Aorta

\section{Fallot-Tetralogie (TOF)}

Die Fallot sche Tetralogie ist durch folgende anatomische Besonderheiten definiert: Durch eine Verlagerung des Outlet-Septums nach anterior und superior kommt es zu einer subvalvulären Pulmonalstenose mit einer unterschiedlich stark ausgeprägten Hypoplasie der Pulmonalklappe und der Pulmonalarterien. Diese Verlagerung des Outlet-Septums führt auch zu einem nicht restriktiven, subaortalen malalignment-Ventrikelseptumdefekt sowie zu einem Überreiten der Aorta über diesem Defekt s. Abb. 7. Die daraus resultierende Druckbelastung 
des rechten Ventrikels hat eine Hypertrophie desselben mit Betonung der Infundibulummuskulatur zur Folge (DGPK: J. Weil et al. 2011).

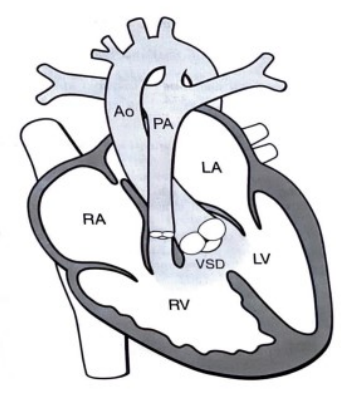

Abb. 7: Fallot-Tetralogie mit subaortalem malalignment-VSD, reitender Aorta, Pulmonalstenose und rechtsventrikulärer Hypertrophie (Abuhamad und Chaoui 2010)

$\mathrm{RA}=$ rechtes Atrium; RV $=$ rechter Ventrikel; $\mathrm{LA}=$ linkes Atrium; $\mathrm{LV}=$ linker Ventrikel; $\mathrm{PA}=$ Pulmonalarterie; Ao = Aorta; VSD = Ventrikelseptumdefekt

\section{Aortenisthmusstenose (Coarctation, ISTA, CoA)}

Bei der Aortenisthmusstenose s. Abb. 8 handelt es sich um eine Einengung der Aorta am Übergang des distalen Aortenbogens in die Aorta descendens (DGPK: N. Haas et al. 2018).

Dieser Herzfehler gehört zu den häufigsten pränatal nicht diagnostizierten Herzfehlern (Detektionsrate $50 \%$ in den ersten 3 Lebensmonaten) (Abdul-Khaliq 2011).

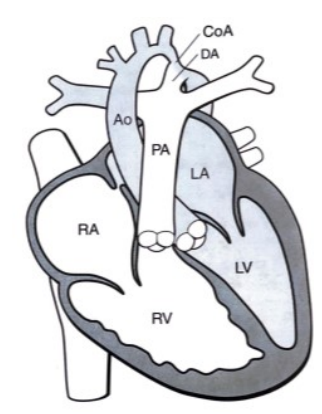

Abb. 8: Aortenisthmusstenose mit Diskrepanz zwischen den Ventrikeln (verengter linker Ventrikel) (Abuhamad und Chaoui 2010)

$\mathrm{RA}=$ rechtes Atrium; $\mathrm{RV}=$ rechter Ventrikel; $\mathrm{LA}=$ linkes Atrium; $\mathrm{LV}=$ linker Ventrikel; $\mathrm{PA}=$ Pulmonalarterie; $\mathrm{Ao}=$ Aorta $; \mathrm{DA}=$ ductus Arteriosus $\mathrm{CoA}=$ Aortenisthmusstenose

\section{Atriumseptumdefekt (ASD I und ASD II)}

Vorhofseptumdefekte im engeren Sinne können als Substanzdefekte mit Blutaustausch zwischen linkem und rechtem Vorhof (Shuntfluss) definiert werden. Dabei können drei 
Haupttypen des ASD (Atrialer Septumdefekt) unterschieden werden: der Ostium secundum Defekt (ASD II, ca. 80\%), der Ostium primum Defekt (ASD I, ca. 10\%) s. Abb. 9 und der Sinus venosus Defekt (DGPK C. Jux et al. 2019).

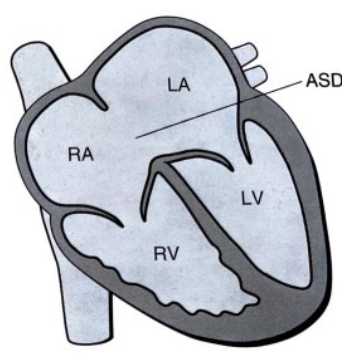

Abb. 9: ASD Typ I (Abuhamad und Chaoui 2010)

$\mathrm{RA}=$ rechtes Atrium; $\mathrm{RV}=$ rechter Ventrikel; $\mathrm{LA}=$ linkes Atrium; $\mathrm{LV}=$ linker Ventrikel

\section{Ventrikelseptumdefekt (VSD)}

Bei einem Ventrikelseptumdefekt besteht eine Kommunikation zwischen dem linken und rechten Ventrikel. Klassifikation und Einteilung erfolgt nach der Häufigkeit der anatomischen Lokalisationen s. Abb. 10:

1. perimembranöser VSD: $80 \%$ - gewöhnlich mit Extension ins muskuläre, Inlet- oder Outlet-Septum.

2. Outlet VSD: 5-7 \% - in enger Lagebeziehung zur Pulmonalklappe

3. Inlet VSD: 5-8 \% - posterior und/oder inferior des membranösen Septums

4. muskuläre Defekte 5-20\% - Lokalisation zentral, apikal, marginal an der Insertion der rechtsventrikulären Vorderwand oder multipel (DGPK M. Gorenflo et al. 2019).

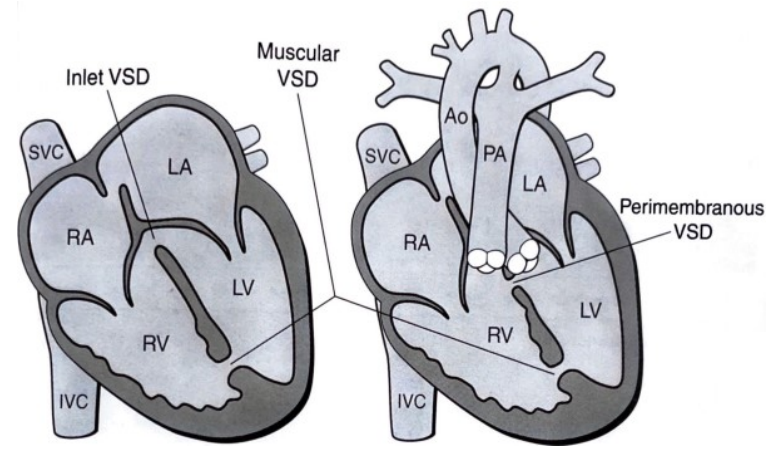

Abb. 10: Ventrikelseptumdefekt (Abuhamad und Chaoui 2010)

$\mathrm{RA}=$ rechtes Atrium; RV = rechter Ventrikel; LA = linkes Atrium; LV = linker Ventrikel; PA = Pulmonalarterie; $\mathrm{Ao}=$ Aorta; $\mathrm{SVC}=$ Vena cava superior; $\mathrm{IVC}=$ Vena cava inferior 


\section{Truncus arteriosus communis (TAC)}

Der TAC ist gekennzeichnet durch ein singuläres arterielles Gefäß (Truncus) mit einer Semilunarklappe (Truncusklappe), welches den Körper-, Lungen- und Koronarkreislauf versorgt, sowie einen Ventrikelseptumdefekt (Konus-Septum-Defekt), über dem der Truncus „reitet“ s. Abb. 11. Die Truncusklappe weist eine unterschiedliche Zahl meist dysmorpher Taschen auf und ist häufig insuffizient. Die Einteilung der Truncustypen erfolgt anhand der Anatomie der Pulmonalgefäße. Aufgrund des großen VSD herrscht in beiden Ventrikeln Druckausgleich. Mit dem Abfall des Lungenwiderstandes nach der Geburt nimmt die Lungendurchblutung stark zu. Bei einer vermehrten Lungendurchblutung unterliegen beide Ventrikel einer erheblichen Volumenbelastung, die unter Umständen durch eine Truncusklappeninsuffizienz noch verstärkt wird. Es kommt oft schon in der Neugeborenenzeit zur schweren Herzinsuffizienz. Der TAC macht unter allen angeborenen Herzfehlern einen Anteil von 0,5-0,8\% aus und zählt damit zu den seltenen konotrunkalen Fehlbildungen (DGPK: N. A. Haas et al. 2020).

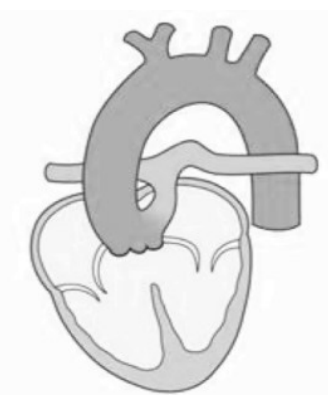

Abb. 11: Beispiel TAC: Aorta und der Stamm der Pulmonalarterie entspringen aus einem gemeinsamen Gefäßstamm (DGPK: N. A. Haas et al. 2020)

\subsubsection{Definition und Epidemiologie}

Die American Heart Association (AHA) definiert angeborene Herzfehler als vor der Geburt bestehende strukturelle Störungen vom Herzen und oder den großen intrathorakalen Blutgefäßen.

Herzfehler sind mit 10:1000 Geburten (Reller et al. 2008, Lindinger et al. 2010) die häufigsten angeborenen Störungen eines einzelnen Organs (Dastgiri et al. 2002, Posada de la Paz und Groft 2010, Mathews und MacDorman 2011).

Die Prävalenz wird in der Literatur unterschiedlich angegeben. Diese reicht von 4-5/1000 
Lebendgeburten (Ferencz et al. 1985) bis zu 12-14/1000 Lebendgeburten in den neueren Veröffentlichungen (Wren et al. 2000, Harris et al. 2003, Lindinger et al. 2010, Liu et al. 2019).

In einer Metaanalyse von 62 Publikationen (Hoffman und Kaplan 2002) wird darauf hingewiesen, dass die Prävalenz der angeborenen Herzfehler vor allem von der relativen Anzahl leichter Herzfehler abhängt, und hier vor allem vom Anteil der gefundenen VSD, welcher den häufigsten angeborenen Herzfehler darstellt.

Außerdem hängt die Prävalenz vom Studiendesign und dabei von der Zusammensetzung des Kollektivs unter dem Aspekt der Risikokonstellationen für einen CHD, wie z.B. dem maternalen Alter, ab. Aber auch die postnatale Pulsoxymetriemessung sowie die physische Untersuchung durch Herzauskultation als Screeninguntersuchung tragen zur Detektion von immer mehr Herzfehlern bei (Götz et al. 2014, Yoon et al. 2020).

Die pränatale Prävalenz von Herzfehlern ist mit der postnatalen jedoch nicht gleichzusetzen, da zum Beispiel der häufigste angeborene Herzfehler VSD nur eine pränatale Erkennungsrate von 30-50\% aufweist (van Velzen et al. 2016) und andererseits Schwangerschaften mit Feten, die einen schweren Herzfehler haben, nicht immer mit einem lebendgeborenen Kind beendet werden (Bhat et al. 2013, Jicinska et al. 2017).

Die steigende Prävalenz pränatal diagnostizierter Herzfehler wird außerdem im Wesentlichen auf die immer besser werdende Ultraschalltechnologie sowie die immer höhere Spezialisierung der Untersucher zurückgeführt (ISUOG: S. Eik-Nes 2006, U. Gembruch et al. 2013). So wurde für die Untersuchung im 2. Trimenon durch die systematische Untersuchung mittels Vierkammerblick und teilweise auch durch die beiden ventrikulären Ausflusstrakte erreicht, dass 60-80\% der schweren Herzfehler („major CHD“), aber auch milde („minor CHD“) Herzfehler, wie kleine Ventrikelseptumdefekte, erkannt werden. Einen entscheidenden Anteil trägt daran der Einsatz von Farb-Doppler-Geräten, verbunden mit einem intensiven Training der Untersucher (U. Gembruch et al. 2013).

In Deutschland erfolgte mit der PAN-Studie 2010 (Prävalenz angeborener Herzfehler bei Neugeborenen) eine bundesweite Erhebung zur Prävalenz von Herzfehlern. Diese schloss 670.000 Geburten ein. Herzfehler traten mit einer Gesamtprävalenz von 1,08 \% auf.

Die Häufigkeitsverteilung der einzelnen Herzfehler stellt sich wie folgt dar:

Ventrikelseptumdefekt (alle Formen): 48,9 \%, Vorhofseptumdefekte: 17,0 \%, valvuläre Pulmonalstenose 6,1\%, persistierender Ductus arteriosus 4,3\%, Aortenisthmusstenose 3,6 \%. Die häufigsten zyanotischen Herzfehler waren Fallot-Tetralogie (2,5 \%) und komplette 
Transposition der großen Arterien (2,2\%). Ein univentrikuläres Herz bestand bei 2,8 \% der Patienten, davon hatten 50 \% ein hypoplastisches Linksherzsyndrom (Lindinger et al. 2010).

Trotz der Fortschritte in Diagnostik und Behandlung der Herzfehler sind diese immer noch der führende Grund für Mortalität und Morbidität im Säuglings - und Kindesalter (Centers for Disease und Prevention 1998, U. Gembruch et al. 2013, Sankar et al. 2016). So bleibt trotz der steigenden Prävalenz von Herzfehlern die Rate an unentdeckten Herzfehlern bei der Geburt bei 50 \% (Hill et al. 2015, van Velzen et al. 2016). Eine Metaanalyse zum Vergleich der Überlebenswahrscheinlichkeit von Feten mit schwerem Herzfehler mit und ohne pränataler Diagnosestellung zeigt, dass die Überlebensrate bis zur geplanten Herz-Operation in der Gruppe mit pränataler Diagnose signifikant höher war (Holland et al. 2015).

Dies macht die Dringlichkeit deutlich, vor allem zyanotische und interventionspflichtige Herzfehler schon pränatal zu diagnostizieren.

\subsubsection{Klassifikation}

Angeborene Herzfehler können nach verschiedenen Kategorien eingeteilt werden. Gängige Einteilungen sind:

- zyanotisch vs. azyanotisch

- ductusabhängig vs. nicht ductusabhängig

- $\quad$ nach Schweregrad bzw. Komplexität in mild, moderat und schwer

Letztere Einteilung ist die übliche Klassifikation in vielen nationalen und internationalen Studien (Connelly et al. 1998; Warnes et al. 2001; Hoffmann und Kaplan, 2002; Chung, 2010 (Warnes et al. 2001, Botto et al. 2003, Lindinger et al. 2010). Die o.g. deutschlandweite Studie zur Prävalenz angeborener Herzfehler, die PAN-Studie (Lindinger et al. 2010), stützt ihre Einteilung der Herzfehler auf die Association for European Paediatric (Coding Committee of the Association for European Paediatric 2002) und teilt angeborene Herzfehler nach ihrer Komplexität wie folgt ein (Auszug):

milde CHD: $\quad$ VSD (klein oder muskulär)

ASD (alle Formen)

valvuläre Pulmonalstenose

leichte Aortenbogenanomalien wie rechtsverlaufender Aortenbogen Trikuspidalklappeninsuffizienz 
Trikuspidalklappendysplasie

aberrante Arteria subclavia rechts

isolierte Mitralklappenstenose

moderate CHD: $\quad$ VSD (andere als mild zugeordnet)

AVSD $=$ atrioventricular septal defect

valvuläre Aortenstenose

Aortenisthmusstenose

partielle Lungenvenenfehlmündung

schwere CHD: $\quad$ UVH = univentrikuläres Herz (alle Typen: DILV, HLHS,

Trikuspidalatresie)

Fallot Tetralogie

Pulmonalatresie mit VSD

Pulmonalatresie mit intaktem Ventrikelseptum

DORV $=$ Double Outlet Right Ventricle

D-TGA $=$ Komplette Transposition der großen Arterien

$\mathrm{TAC}=$ Truncus arteriosus communis

IAA $=$ interrupted aortic arch $=$ unterbrochener Aortenbogen

totale Lungenvenenfehlmündung

Ebstein-Anomalie

Auch die ESC (European Society of Cardiology) teilt angeborene Herzfehler in ihren aktuellen Guidelines zum Management angeborener Herzfehler bei Erwachsenen 2020 (Baumgartner et al. 2020) nach ihrer Komplexität dementsprechend ein.

Ergänzt werden sollte diese Einteilung noch um die isolierte LSVC, die zu den milden Herzfehlern gezählt werden kann (Abuhamad und Chaoui 2010), sowie die Kardiomyopathien, die je nach Ausprägung zu den schweren oder moderaten Herzfehlern zählen (Dittrich et al. 2012). Ectopia cordis ist in gängigen Klassifikationen oft nicht erwähnt, kann aber aufgrund der schwerwiegenden Konsequenzen (Pius et al. 2017) dieser Fehlbildung ebenfalls zu den schweren Herzfehlern gezählt werden. 
Von allen Herzfehlern entfallen circa zwei Drittel auf die milden Formen und ein Drittel auf die moderaten und schweren Formen (S. Dittrich et al. 2019). Vom angeborenen Herzfehler sind die Arrhythmien ohne strukturelles Korrelat sowie die Persistenz des Ductus botalli und des Foramen ovale abzugrenzen (Mitchell et al. 1971).

\subsection{4 Ätiologie und Risiken}

Am häufigsten treten Herzfehler bei Feten ohne bekannte Risikofaktoren auf (Allan 1995, Sharland 2004, U. Gembruch et al. 2013). Trotzdem wird die fetale Herzuntersuchung vor allem in identifizierbaren Risikogruppen durchgeführt. Dieses Risikoprofil setzt sich aus genetischen, epigenetischen, maternalen, fetalen sowie Umweltfaktoren zusammen. Diese Risikofaktoren können sich gegenseitig potenzieren und damit die kausale Betrachtung einzelner Assoziationen erschweren (Ferencz und Boughman 1993, Ahmadi et al. 2020).

\subsubsection{Maternale Risikofaktoren}

Mütterliche Stoffwechselerkrankungen haben einen signifikanten Einfluss auf die Entstehung eines CHD (Abuhamad und Chaoui 2010). Dazu zählen der mütterliche Diabetes mellitus, der das Risiko für einen fetalen Herzfehler um das Fünffache erhöhen kann (Rowland et al. 1973). Hierbei ist vor allem ein unzureichend eingestellter Blutglucose-Wert im ersten Trimester entscheidend.

Auch Adipositas ab einem BMI $\geq 30 \mathrm{~kg} / \mathrm{m}^{2}$ wird mit Herzfehlern assoziiert (Cedergren und Kallen 2003, Brite et al. 2014, Persson et al. 2017). Einerseits wird die häufig mit einer Adipositas vergesellschaftete gestörte Glukosetoleranz in der Frühschwangerschaft als Mechanismus für die Entstehung von Herzfehlern diskutiert. Andererseits werden vom Fettgewebe ausgehende hormonelle Einflüsse auf die Expression bestimmter Gene als Faktor für die Entwicklung von Herzfehlern vermutet (Cedergren und Kallen 2003, Zhu et al. 2010, Persson et al. 2017).

Zudem führt eine unbehandelte Phenylketonurie bei Müttern mit erhöhten Phenylkentonuriespiegeln zu einem 6-fach erhöhten Risiko für Herzfehler (Levy und Waisbren 1983).

Eine weitere Assoziation wird zwischen Medikamenten und der Organogenese des Herzens gesehen. Dazu zählen Lithium, Antikonvulsiva, Isorethinoin, Indometacin, ACE-Hemmer sowie SSRIs (Abuhamad und Chaoui 2010). 
Außerdem steigt mit zunehmendem mütterlichen Alter auch bei Feten ohne chromosomale Störungen das Risiko für angeborene Herzfehler (Jenkins et al. 2007, Miller et al. 2011, Pavlicek et al. 2011).

Weitere gut belegte Risikofaktoren sind Rauchen im ersten Trimenon (Alverson et al. 2011) sowie der Konsum von Alkohol (Sun et al. 2015). Bei einem fetalen Alkoholsyndrom finden sich bei bis zu 25-30 \% der Kinder ein CHD (Abuhamad und Chaoui 2010).

Bei Kindern, die durch Reproduktionstechnologie wie IVF und ISCI entstehen, ist ein bis zu vierfach erhöhtes Risiko für CHD beschrieben (Hansen et al. 2005, Tararbit et al. 2013).

Feten, bei denen es eine positive Familienanamnese bezüglich eines Herzfehlers gibt, haben ebenfalls ein erhöhtes Risiko, an einem Herzfehler zu leiden, vor allem wenn mehrere Familienmitglieder betroffen sind oder es sich um Verwandte ersten Grades handelt. Hier erhöht sich das Risiko von 1 \% für die Allgemeinbevölkerung auf 2-4 \% (Fung et al. 2013). Weiterhin können Infektionskrankheiten wie Rötel-, Herpes- oder Zytomegalievirusinfektion, vor allem in den ersten drei Monaten der Schwangerschaft, Ursache für einen Herzfehler sein (Konorza und J. Forkel n.d.).

\subsubsection{Fetale Risikofaktoren}

Fetale Risikofaktoren sind genetische Störungen oder sonomorphologische Auffälligkeiten, die mit Herzfehlern assoziiert sein können. Dazu zählen chromosomale Störungen und eine verdickte Nackenfalte (s.u.), extrakardiale Anomalien, fetale Arrhythmien und eine fetale Wachstumsrestriktion. Zudem haben Mehrlinge mit monochorialer Plazentation ein erhöhtes Risiko für das Vorliegen eines Herzfehlers (Abuhamad und Chaoui 2010).

Findet sich in der fetalen Untersuchung eine anatomische Malformation, wie z.B. ein nichtimmunologischer Hydrops fetalis, liegt zu 15-20 \% ein Herzfehler vor. Das Risiko für einen CHD steigt, wenn mehrere Organsysteme von einer Fehlbildung betroffen sind oder eine Assoziation mit einer genetischen Störung vorliegt. So haben ca. 50-70 \% der Feten mit CHD und chromosomalen Anomalien andere assoziierte extrakardiale Anomalien (Copel et al. 1988, Eydoux et al. 1989). Die häufigsten Fehlbildungen, die mit einem CHD assoziiert werden, sind laut Abuhamad und Chaoui (Abuhamad und Chaoui 2010) folgende: s. Tab 1. 
Tab. 1 CHD assoziierte Fehlbildungen

\begin{tabular}{|l|l|}
\hline Extrakardiale Malformation & $\begin{array}{l}\text { Inzidenz der CHD } \\
\text { Assoziation }\end{array}$ \\
\hline Bilaterale Nierenagenesie & $42,8 \%$ \\
\hline Hufeisenniere & $38,8 \%$ \\
\hline Omphalozele & $19,5 \%$ \\
\hline Duodenalatresie & $17,2 \%$ \\
\hline Unilaterale Nierenagenesie & $16,9 \%$ \\
\hline Corpus-callosum-Agenesie & $14,8 \%$ \\
\hline Tracheoösophageale Fistel & $14,7 \%$ \\
\hline Analatresie & $11,7 \%$ \\
\hline Nicht-immunologischer & $10-20 \%$ \\
\hline Hydrops & $8-9 \%$ \\
\hline Singuläre Umbilikalarterie & \\
\hline
\end{tabular}

Unter den HRST hat der AV-Block III mit $40 \%$ die höchste Assoziation zu einer strukturellen Herzerkrankung. Bei fetalen Tachykardien (>180 bpm) ist bei $5 \%$ mit einem Vitium cordis zu rechnen (Dietrich 2006).

Zudem können im Rahmen des Aneuplodiescreenings aufgefallene sonografische Marker wie eine erhöhte Nackentransparenz (s.u.), ein abnormes Blutflussmuster im Ductus venosus oder das Vorliegen einer Trikuspidalklappenregurgitation Hinweiszeichen auf das Vorliegen eines Herzfehlers sein (Clur und Bilardo 2014). Dies sollte zu einer erweiterten echokardiographischen Untersuchung führen. Vor allem der abnorme DV-Fluss und die TR eignen sich durch ihr transientes Auftreten mit einer hohen Falsch-Positiv-Rate und geringen positiven Vorhersagewerten jedoch nicht als Screening für CHD (Geipel und Gembruch 2012).

Die Nackentransparenz beschreibt eine Flüssigkeitsansammlung im Bereich des fetalen Nackens. Diese ist in den frühen Schwangerschaftswochen stets nachzuweisen und somit bis $\mathrm{zu}$ einem bestimmten Maß als normal anzusehen. Mit Zunahme der Größe der 
Nackentransparenz steigt jedoch das Risiko für mögliche Anomalien des Feten (Voigt et al. 2018). Bei auffälligen NT Messwerten sollte eine weiterführende Ultraschalluntersuchung auf DEGUM Level II/III durchgeführt werden.

Die Nackenfaltenmessung ist eine etablierte Methode zur Risikobewertung für das Vorliegen einer Trisomie 21, 13 oder 18 (Nicolaides et al. 1992) s. Kapitel 3.2.2.

Seit Mitte der 90er Jahre ist außerdem der Zusammenhang mit fetalen Herzfehlern, auch ohne das Vorliegen einer chromosomalen Störung, bekannt (Hyett et al. 1996).

Das Risiko für das Vorliegen eines Herzfehlers steigt dabei exponentiell mit der Dicke der NT. Bei Feten mit einer Nackentransparenz $>95$. Perzentile findet man zu ca. $44 \%$ einen schweren Herzfehler (Clur et al. 2009). So ergab eine Metaanalyse von 10 Studien mit 192.922 euploiden Feten, dass das Risiko eines Herzfehlers in der Gruppe zwischen 2,5 und 3,4 mm Nackendicke um das 2,5- Fache und in der Gruppe mit einer NT $\geq 3,5 \mathrm{~mm}$ um das 10-fache steigt, verglichen mit einer normalen Prävalenz für CHD von 8/1000 Lebendgeborenen (Clur et al. 2009).

Die einzelnen Herzfehler scheinen sich jedoch nicht bezüglich ihrer NT-Werte zu unterscheiden (Makrydimas et al. 2005).

\subsubsection{Syndromale Herzfehler}

In vielen Studien wurde gezeigt, dass die Rate an detektierten Herzfehlern höher ist, wenn diese mit einem Syndrom assoziiert oder mit einer Single-Gene Disorder (Defekte an einzelnen Genen) verbunden sind (Friedberg et al. 2009, Atz et al. 2010).

Ca. 5-15\% der Kinder mit Herzfehlern haben eine chromosomale Störung (Ferencz et al. 1989, Harris et al. 2003, S. Dittrich et al. 2019).

Typische mit Herzfehlern assoziierte genetische Störungen sind in Tab. 2 aufgeführt. 
Tab. 2: Typische mit Herzfehlern assoziierte genetische Störungen - Auszug aus (Pierpont et al. 2007)

\begin{tabular}{|c|c|c|}
\hline Chromosomale Störung & $\begin{array}{l}\text { Assoziation } \\
\text { mit } \\
\text { Herzfehlern }\end{array}$ & Herzanomalie \\
\hline $\begin{array}{l}\text { Trisomie } 13 \text { (Pätau- } \\
\text { Syndrom) }\end{array}$ & $80 \%$ & $\begin{array}{l}\text { ASD, VSD, PDA, HLHS, CoA, Lateralitäts- } \\
\text { Defekte }\end{array}$ \\
\hline $\begin{array}{l}\text { Trisomie } 18 \text { (Edwards- } \\
\text { Syndrom) }\end{array}$ & $90-100 \%$ & ASD, VSD, PDA, TOF, DORV, CoA, u.v.m \\
\hline Trisomie 21 & $40-50 \%$ & AVSD, VSD, ASD, TOF \\
\hline $\begin{array}{l}\text { Monosomie X (Turner- } \\
\text { Syndrom } 45 \text { X) }\end{array}$ & $25-35 \%$ & CoA, valvuläre AS, HLHS, Aortendissektion \\
\hline $\begin{array}{l}\text { Klinefelter Syndrom }(47 \\
\text { XXY) }\end{array}$ & $50 \%$ & PDA, ASD, MVP \\
\hline $\begin{array}{l}\text { Deletion 22q11 (DiGeorge- } \\
\text { Syndrom) }\end{array}$ & $75 \%$ & $\begin{array}{l}\text { Unterbrochener Aortenbogen, TAC, isolierte } \\
\text { Aortenbogenanomalie, TOF, VSD }\end{array}$ \\
\hline Mosaik-Trisomie 8 & $25 \%$ & VSD, PDA, CoA, TAPVC, TAC \\
\hline Trisomie 9 & $65-80 \%$ & PDA, LSVC, VSD, TOF, DORV \\
\hline
\end{tabular}

Außerdem zählen zu den Einzelgendefekten, die mit dem Auftreten von angeborenen Herzfehlern assoziiert sind, unter anderem das Holt-Oram-, das Noonan- und das MarfanSyndrom.

Auch können Herzfehler nicht-syndromal mit Einzelgendefekten verbunden sein (z.B. dTGA, DORV mit dem Gen CFC1 auf dem Chromosom 2q21) (Pierpont et al. 2007).

\subsubsection{Prävention}

Prävention angeborener Herzfehler beginnt im Erkennen und Vermeiden der Risikofaktoren, die als CHD-assoziiert gelten und die Schwangerschaft vor allem perikonzeptionell und bis zum Abschluss der embryonalen Herzentwicklung 2-7 SSW p.c. beeinflussen.

So führt die AHA nach einem Review über bekannte Risikofaktoren (Jenkins et al. 2007) folgende Empfehlungen für die Prävention angeborener Herzfehler auf:

Frauen mit Kinderwunsch sollten 
- Folsäure supplementieren

- Vorsorgeuntersuchungen wahrnehmen, mit besonderem Augenmerk auf die Erkennung und Behandlung von Diabetes, Phenylketonurie sowie Impfung gegen Röteln

- Medikamente (auch rezeptfreie) nur nach ärztlicher Rücksprache einnehmen

- Kontakt zu Menschen mit Grippe oder anderen fieberhaften Infekten vermeiden

- Exposition gegenüber organischen Lösungsmitteln vermeiden

\subsubsection{Perinatales Management}

In Deutschland werden in etwa 6000-8000 Kinder mit Herz- oder Gefäßanomalien lebend geboren. Ca. 50 \% werden den schweren kardialen Anomalien zugeordnet und bedürfen einer operativen Therapie. Ist ein Herzfehler bereits pränatal erkannt, kann die Geburt in einem interdisziplinären Zentrum geplant werden und das herzkranke Kind bekommt ohne Zeitverzögerung die notwendige Betreuung und Therapie. Neben intensivmedizinischen und medikamentösen Behandlungsmöglichkeiten ist in vielen Fällen auch die Möglichkeit einer frühzeitigen interventionellen und/oder chirurgischen Therapie für die Prognoseverbesserung der herzkranken Neugeborenen wichtig (Bull 1999, Meyer-Wittkopf und Hofbeck 2003).

Ist der Herzfehler eines Neugeborenen nicht bekannt, sind die zu erwartenden Symptome je nach vorliegendem Herzfehler sehr unterschiedlich. Diese reichen von lediglich zufällig entdecktem Herzgeräusch und Auffälligkeiten in der Oxymetrie bis zu einer schweren Zyanose, vermehrtem Schwitzen, Kurzatmigkeit, Tachykardie und Nahrungsverweigerung (Sun et al. 2015).

Um den Herzfehler postnatal weiter zu differenzieren, stehen verschiedene diagnostische Möglichkeiten zur Verfügung. Dazu zählen unter anderem die Pulsoxymetrie, EKG, Blutdruckmessung, Echokardiographie, CT, MRT, Röntgenaufnahmen des Thorax sowie die Untersuchung mittels Herzkatheter. Durch diese additiven Methoden werden genaue diagnostische Aussagen getroffen. Die gewonnenen Untersuchungsergebnisse können Einfluss auf die Therapieplanung haben.

Bedarf ein angeborener Herzfehler einer Behandlung, gibt es verschiedene Strategien: eine medikamentöse Behandlung, die chirurgische Intervention und/oder eine Katheterintervention (NHLBI USA 2014). Auch können die Behandlungsoptionen kombiniert werden. Zum Beispiel muss bei einer Transposition der großen Gefäße der für eine ausreichende Oxygenierung notwendige Links-Rechts-Shunt (durch PDA und PFO) bis zur SwitchOperation, welche meist innerhalb der ersten zwei Lebenswochen angestrebt wird, 
offengehalten werden. Dafür erfolgt bei Neugeborenen mit Ductusabhängigkeit eine Prostaglandininfusion. Führt dies nicht $\mathrm{zu}$ einer Verbesserung der systemarteriellen Oxygenierung oder verzögert sich die Operation zum Beispiel durch eine Infektion, erfolgt die Ballonatrioseptostomie (BAS) mittels Herzkatheter (DGPK: C. Rickers 2013).

Dieses beispielhafte Vorgehen macht die Dringlichkeit der pränatalen Diagnostik des vorliegenden Herzfehlers deutlich. Die Geburt eines Kindes mit schwerem Herzfehler in einem Level-I-Perinatalzentrum mit kinderkardiologischer und kinderherzchirurgischer Abteilung ist eine entscheidende Voraussetzung für die Verminderung der Mortalität und Morbidität, weil hierdurch die besten Bedingungen für das Gelingen der postnatalen Therapie geschaffen werden.

\subsection{Ultraschall Untersuchungen}

Im Rahmen der Mutterschaftsvorsorge erfolgen 3 Ultraschalluntersuchungen

1. Screening $8+0$ bis $11+6 \mathrm{SSW}$

2. Screening $18+0$ bis $21+6 \mathrm{SSW}$

3. Screening $28+0$ bis $31+6 \mathrm{SSW}$

In der ersten Screeninguntersuchung werden unter anderem die Frage nach einer positiven Herzaktion sowie die Frage nach „Auffälligkeiten“ mit ja oder nein beantwortet.

Ergeben sich hier Auffälligkeiten oder wünscht die Schwangere eine additive Untersuchung, kann eine frühe Feindiagnostik (frühe Fehlbildungsdiagnostik) und/oder ein Ersttrimesterscreening erfolgen.

\subsubsection{Die frühe Fehlbildungsdiagnostik}

Die frühe Fehlbildungsdiagnostik, auch frühe Feindiagnostik (weiterführende differenzierte Ultraschalluntersuchung in der pränatalen Diagnostik) genannt, ist die Grundlage für das Ersttrimesterscreening, weitere genetische Screeningtests oder ein Präeklampsiescreening s. Tab 3. Die Untersuchung wird nach Empfehlung der DEGUM durchgeführt und beinhaltet die in Abb. 12 aufgeführten Standardeinstellungen und optionalen Parameter. Die Empfehlung richtet sich dabei an DEGUM Stufe II-III qualifizierte Untersucher. Diese Qualifizierung erfolgt in Deutschland im Mehrstufenkonzept der DEGUM und teilt sich in 3 Stufen. Ab DEGUM Stufe II sind die Untersuchenden zur Detektion von Fehlbildungen in speziellen Risikokollektiven mittels weiterführender differenzierter pränataler Diagnostik befähigt (Deutsche Gesellschaft für Ultraschall in der Medizin e.V. n.d.). 


\begin{tabular}{|c|c|c|}
\hline & Standardparameter & optionale Parameter \\
\hline $\begin{array}{l}\text { Schädel/ } \\
\text { Gehirn }\end{array}$ & $\begin{array}{l}\text { Kalotte, Falx cerebri, } \\
\text { Plexus chorioidei }\end{array}$ & $\begin{array}{l}\text { Intrakranielle Transparenz IT } \\
\text { Hirnstamm }\end{array}$ \\
\hline Gesicht & Profil & Augen, Kiefer, Lippen \\
\hline Nacken & Nackentransparenz (NT) ${ }^{1}$ & Nasenbein $(\mathrm{NB})^{1}$ \\
\hline $\begin{array}{l}\text { Wirbel- } \\
\text { säule }\end{array}$ & & Kontur \\
\hline $\begin{array}{l}\text { Herzl } \\
\text { Thorax }\end{array}$ & $\begin{array}{l}\text { Lage, Kontur } \\
\text { Vierkammerblick } \\
\text { Lungen }\end{array}$ & $\begin{array}{l}\text { Ausflusstrakte in Farbe } \\
\text { Drei-Gefäß-Trachea-Blick } \\
\text { Trikuspidalklappenfluss (TR) }{ }^{1}\end{array}$ \\
\hline $\begin{array}{l}\text { Abdo- } \\
\text { men }\end{array}$ & $\begin{array}{l}\text { Magen } \\
\text { Bauchwand }\end{array}$ & $\begin{array}{l}\text { Zwerchfell } \\
\text { Ductus venosus Fluss (DV) }{ }^{1} \\
\text { NS Arterien bds. der Harnblase }\end{array}$ \\
\hline $\begin{array}{l}\text { Extremi- } \\
\text { täten }\end{array}$ & Arme und Beine & $\begin{array}{l}\text { Hände und Füße } \\
\text { (Femur Tibia Fibula Humerus } \\
\text { Radius Ulna) }\end{array}$ \\
\hline $\begin{array}{l}\text { Urogeni- } \\
\text { taltrakt }\end{array}$ & Harnblase & Nieren \\
\hline Plazenta & $\begin{array}{l}\text { Chorionizität, Amnionizi- } \\
\text { tät (Mehrlinge), Struktur }\end{array}$ & $\begin{array}{l}\text { Lage, } \\
\text { Ansatz der Nabelschnur } \\
\text { Aa. uterinae }{ }^{1}\end{array}$ \\
\hline
\end{tabular}

Abb. 12: Sonografische Standardeinstellungen der fetalen Anatomie- und optionale Parameter der FMF (von Kaisenberg et al. 2016)

\subsubsection{Das Ersttrimesterscreening}

Das ETS wird in der 11+0 bis 13+6 SSW durchgeführt und wie folgt definiert:

„Das Ersttrimester-Screening ist eine Risikoanalyse für Chromosomenanomalien und eine Reihe anderer fetaler Fehlbildungen, die bei korrekter sonografischer und biochemischer Anwendung eine hohe Erkennungswahrscheinlichkeit aufweist" (FMF Deutschland e.V. n.d.) s. Tab. 3.

Vor der Durchführung des Ersttrimesterscreenings erfolgt nach dem zugrunde liegenden Gendiagnostik- Gesetz (GenDG) ein Beratungsgespräch zu den Inhalten der Untersuchung und den sich daraus möglicherweise ergebenden Konsequenzen (von Kaisenberg et al. 2016). Es gibt allerdings weder von der FMF London noch von der FMF Deutschland klare Kriterien für die sonografische Untersuchung des Feten und insofern auch des fetalen Herzens im Rahmen des Ersttrimesterscreenings. Somit ist der Umfang der fetalen Organuntersuchung im Rahmen des ETS abhängig von der Qualifikation der Untersucher. 
Tab.3: Nomenklatur der Screening-Untersuchungen im 1. Trimenon (Kozlowski et al. 2019)

\begin{tabular}{|l|l|l|l|}
\hline Untersuchung & Ultraschall-Parameter & $\begin{array}{l}\text { Serum- } \\
\text { Parameter }\end{array}$ & Zielsetzung \\
\hline ETS & NT & Frientierender \\
kombiniertes ETS & NT & $\begin{array}{l}\text { Fehlbildungsausschluss } \\
\text { PAPeuploidie-Screening }\end{array}$ \\
\hline $\begin{array}{l}\text { kombiniertes ETS mit } \\
\text { Markern }\end{array}$ & NT, NAB, DV, TK & $\begin{array}{l}\text { Freies } \beta-H C G \\
\text { PAPP-A }\end{array}$ & $\begin{array}{l}\text { Primäre oder } \\
\text { sekundäre Präzisierung } \\
\text { des ETS-Befundes }\end{array}$ \\
\hline Contingent Screening & $\begin{array}{l}\text { Erweitertes Screening in Abhängigkeit vom Befund des kombinierten } \\
\text { ETS }\end{array}$ & Publizierte Qualitätsanforderungen: DEGUM, ISUOG, FMF \\
\hline Fehühe & \multicolumn{2}{|l|}{} \\
\hline
\end{tabular}

„,contingent screening“: der Begriff wird zunehmend auch für den Einsatz des cfDNA-Screenings nach vorheriger Risikoklassifikation mittels kombinierten ETS verwendet.

Besteht die Indikation bzw. der Wunsch zur Risikoabschätzung einer fetalen Chromosomenstörung oder einer fetalen Fehlbildung, erfolgt im Rahmen des kombinierten Ersttrimesterscreenings die Beurteilung des individuellen Aneuplodierisikos auf die häufigsten Chromosomenaberrationen (Trisomie 13, 18, 21), basierend auf dem maternalen Alter, der Messung der NT sowie der Bestimmung zweier maternaler Serummarker: freies ßhCG (free-ß-human chorionic gonadotropin) und PAPP-A (pregnancy associated plasma protein- A). So ergibt sich auch unter Einbeziehung weiterer Parameter, wie maternalem Gewicht, ethnischer Herkunft und aktuellem Nikotinabusus, ein individuelles Risiko für diese Schwangerschaft. Bei erhöhtem Risiko sollte der Schwangeren eine Chromosomenanalyse mittels diagnostischer Punktion (Fruchtwasserpunktion oder Chorionzottenbiopsie) angeboten werden (Rempen 2001). Zudem steht in Deutschland das im Jahr 2012 eingeführte „Non-Invasive Prenatal Testing“ (NIPT) zur Verfügung. Dieser Test basiert auf der Untersuchung der zellfreien DNA (cfDNA) im mütterlichen Blut und bietet eine Screeninguntersuchung auf die o.g. numerischen Chromosomenstörungen sowie gonosomale Chromosomenstörungen und je nach Testverfahren auch Triploidie an (Voigt et al. 2018, Kozlowski et al. 2019). 


\subsubsection{Die frühe fetale Herzuntersuchung (11+0 - 13+6 SSW)}

\subsubsection{Indikation}

Die Indikation zur frühen fetalen Herzuntersuchung wird einerseits aus Auffälligkeiten in den Screeninguntersuchungen im ersten Trimenon gestellt und andererseits aus dem maternalem und fetalem Risikoprofil.

Hierbei unterscheidet sich die Indikation zur frühen fetalen Echokardiographie im Vergleich zur Echokardiographie im zweiten Trimenon nicht wesentlich. Die ISOUG (ISUOG: S. EikNes 2006) leitet aus der Zusammenschau fetaler und maternaler Risikofaktoren folgende typische Indikationen für die fetale Echokardiographie ab:

Maternale Indikation: Familienanamnese: betroffene erstgradige Verwandte; präexistente Stoffwechselerkrankungen: Diabetes, Phenylketonurie; maternale Infektionen: Parvovirus B19, Röteln, Coxsackie Virus; Exposition mit kardialen Teratogenen: Retinoide, Phenytoin, Carbamazepin, Lithium, Valproinsäure; maternale Antikörper: anti-Ro (SSA) anti-La (SSB).

Fetale Indikation: vermuteter Herzfehler, fetale Aneuploidie, schwerwiegende extrakardiale Fehlbildung, erhöhte NT ( $\geq 3.5$ mm vor $14+0$ SSW), fetale Herzfrequenz- oder Rhythmusstörung sowie Wachstumsretardierung.

Die im fetalen Risikoprofil enthaltenen transienten Hinweiszeichen für das Vorliegen eines Herzfehlers, wie eine erhöhte Nackentransparenz, ein abnormaler Ductus-venosus-Fluss oder eine Trikuspidalregurgitation, identifizieren eine Risikogruppe, die eine frühe Untersuchung des fetalen Herzens erhalten sollte, s. Kapitel 3.1.4.2.

Da jedoch die Mehrzahl aller Feten mit Herzfehlern weder ein familiäres Risiko noch Auffälligkeiten in der frühen Feindiagnostik aufweisen, wird in der Literatur die generelle Durchführung einer frühen fetalen Echokardiographie diskutiert (Volpe et al. 2011, Becker et al. 2012). Dies ist aber wirtschaftlich und organisatorisch mit hohen Anforderungen verbunden. Zudem ist die alleinige Einstellung des $4 \mathrm{~KB}$ mit niedrigen Entdeckungsraten für Herzfehler assoziiert. In Niedrigrisiko-Kollektiven und durchgeführt durch weniger erfahrene Untersucher, werden Herzfehler nur zu $11 \%$ mittels 4KB erkannt (Westin et al. 2006). Die fetale Echokardiographie ist wiederum den hoch spezialisierten Untersuchern und Zentren vorbehalten. Daher ist es notwendig, ein Protokoll für die frühe kardiale Untersuchung zu 
etablieren, welches mehr Informationen als die alleinige Einstellung des Vierkammerblicks enthält, aber nicht so aufwendig wie eine ausführliche fetale Echokardiographie ist.

\subsubsection{Vierkammerblick, rechtsventrikuläre und linksventrikuläre Ausflussbahn}

Eine Möglichkeit zu diesem o.g. Protokoll stellt die feste Kombination von 4KB, LVOT und RVOT unter Einsatz von Farbdoppler dar, welche in der frühen FD nur als optional angesehen wird s. Abb. 12.

\section{Vierkammerblick mit Farbdoppler}

- Vorhandensein und Größenverhältnis beider Herzhälften

- Größe und Lage des Herzens im Thorax

- Beurteilung des AV Klappenflusses

- Kontinuität des Ventrikelseptums (Berg et al. 2007)

\section{LVOT (left Ventricular Outflow Tract) mit Farbdoppler}

- Abgang einer großen Arterie aus dem LV

- Fluss über der Aortenklappe (Beweglichkeit)

\section{RVOT (right Ventricular Outflow Tract) mit Farbdoppler}

- Abgang einer großen Arterie aus dem RV

- Fluss über der Pulmonalklappe (Beweglichkeit) (ISUOG: S. Eik-Nes 2006)

Demzufolge wären die erkennbaren Anomalien:

- Univentrikuläre Situation

- Größendifferenz der Kammern

- $\quad$ große VSD / AVSD

- Anomalien im Bereich der Ausflusstrakte: Darstellung eines singulären Gefäßes, Malposition der Gefäße, Größendifferenz der Gefäße, Turbulenzen o.a. abnormaler Fluss über den Semilunarklappen, retrograde Perfusion

Beispielhaft sehen erhobene Normalbefunde der drei kardialen Einstellungen wie in Abb. 13 -15 dargestellt aus. 


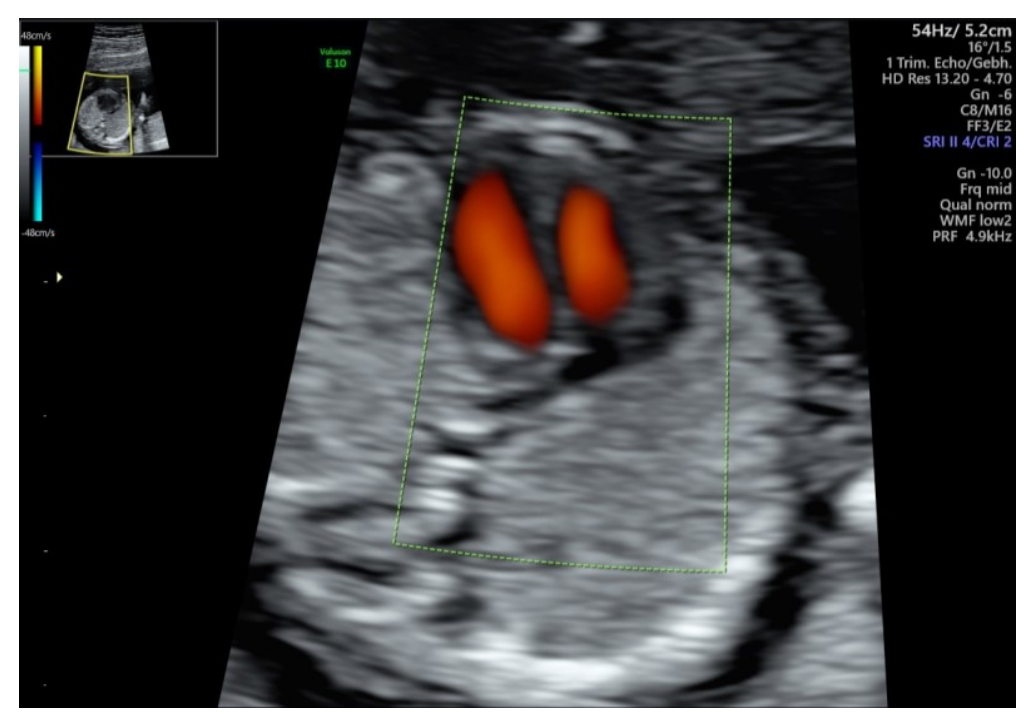

Abb. 13: Normalbefund 4KB mit Farbdoppler

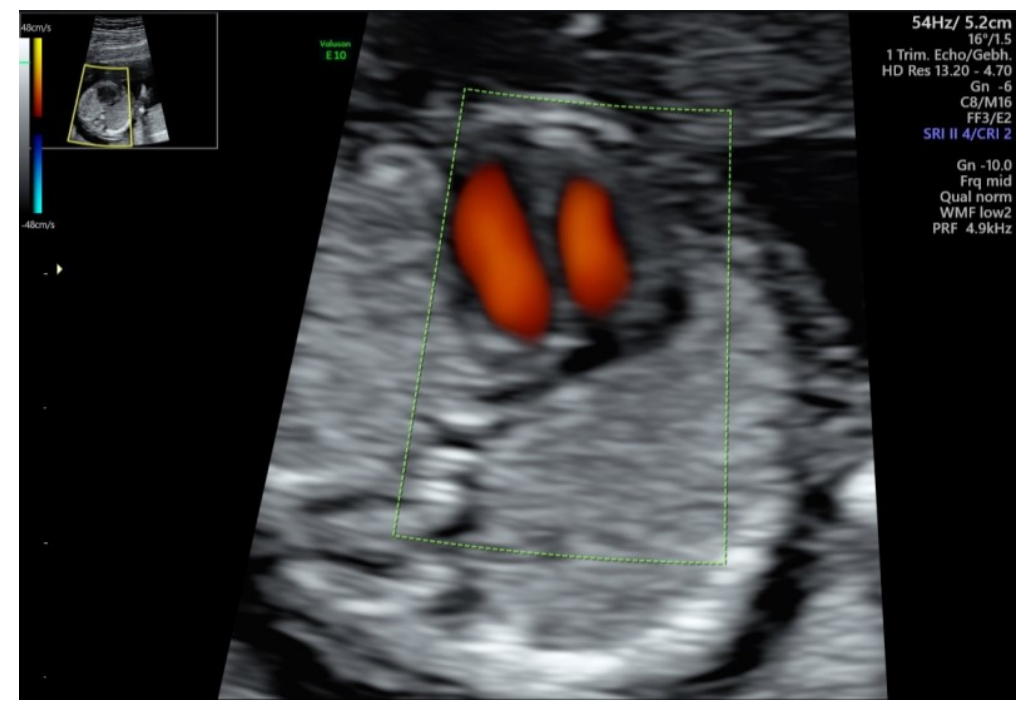

Abb. 14: Normalbefund LVOT mit Farbdoppler

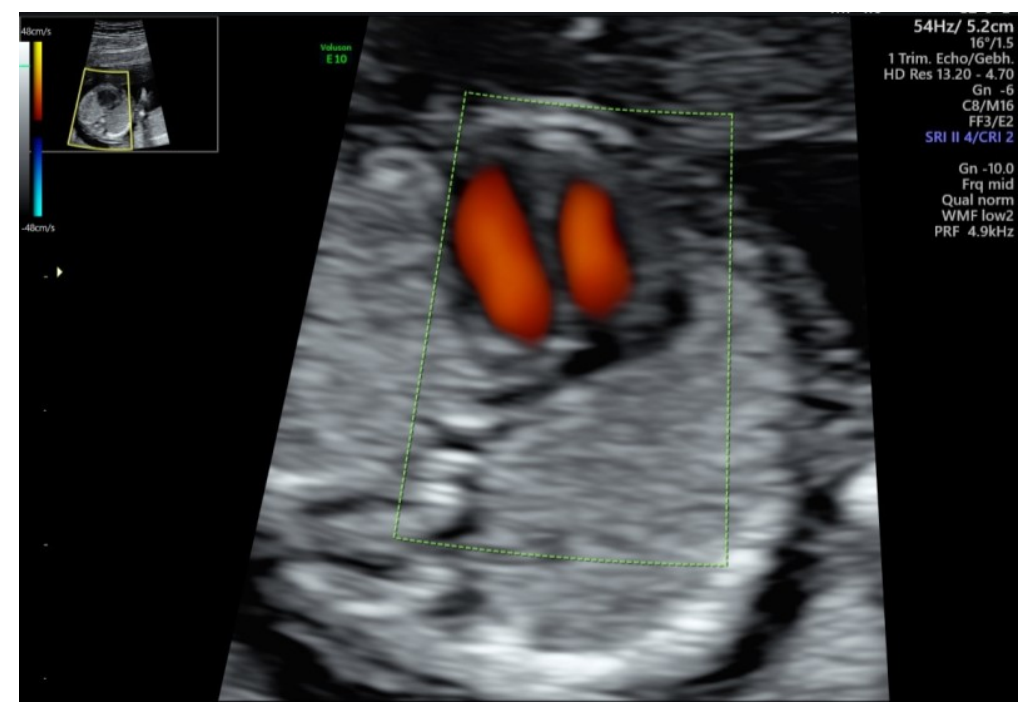

Abb. 15: Normalbefund RVOT mit Farbdoppler 
Die drei Einstellungen 4KB, LVOT und RVOT können in allen genannten Risikogruppen der schnellen Orientierung dienen, ob eine fetale Echokardiographie durchgeführt werden sollte. Somit stellen diese Einstellungen eine Schnittstelle im Rahmen der frühen Feindiagnostik zur Herzbeurteilung dar, jedoch ohne dieselben hohen personellen, apparativen und zeitaufwendigen Anforderungen wie eine frühe fetale Echokardiographie zu haben.

Im ersten Trimenon werden bei vollständiger echokardiografischer Untersuchung unter Einbeziehung der Ausflusstrakte Entdeckungsraten zwischen 63\% und 70\% angegeben (Smrcek et al. 2006). Dabei betreffen die publizierten Studien jedoch überwiegend Daten zu Hoch-Risiko-Kollektiven, in denen Feten mit erweiterter Nackentransparenz und/oder Aneuploidie überrepräsentiert sind.

Es sind nur wenig aktuelle Daten zur Entdeckungsrate fetaler Herzfehler durch die Einstellung von $4 \mathrm{~KB}$, LVOT und RVOT im ersten Trimester publiziert (Becker und Wegner 2006, Hernandez-Andrade et al. 2017).

Die hier vorliegende Arbeit untersucht diese Fragestellung. Außerdem hängt die Entdeckungsrate fetaler Herzfehler wie im zweiten Trimenon in erster Linie vom Ausbildungsstand des Untersuchers, von der Lage des Fetus, Untersuchungsbedingungen sowie der Komplexität der Untersuchung ab (Meyer-Wittkopf 2003, Berg et al. 2007).

\subsubsection{Die fetale Echokardiographie im zweiten Trimenon}

Die Feindiagnostik im 2. Trimenon $(18+0$ bis $21+6)$ beinhaltet bezüglich der Herzbeurteilung folgende in Abb. 16 aufgeführten Parameter:

\begin{tabular}{ll|}
\hline Thorax: & Lungen: Struktur \\
& Herz: Herzfrequenz und -rhythmus, qualitative Ein- \\
& schätzung von Größe, Form und Position des Herzens, \\
\hline & Vierkammerblick, links- und rechtsventrikulärer Aus- \\
& flusstrakt \\
\hline & Zwerchfell: Kuppelkontur im Sagittalschnitt
\end{tabular}

Abb. 16: zu überprüfende sonoanatomische Parameter im Zeitraum von $18+0$ bis $21+6$ SSW im Rahmen der weiterführenden differentialdiagnostischen Ultraschalluntersuchung (Merz et al. 2012) 
Anders als in der Ersttrimesteruntersuchung ist die Beurteilung der Ausflussbahnen zusätzlich zum 4-Kammerblick obligater Bestandteil der Untersuchung (Carvalho et al. 2002). Gibt es Hinweise auf einen fetalen Herzfehler, sollte eine fetale Echokardiographie erfolgen.

Die DEGUM (Chaoui et al. 2008) empfiehlt zur Durchführung der Echokardiographie Folgendes zu dokumentieren

- Querschnitt des oberen Abdomens

- Vierkammerblick (B-Bild und Farbdoppler)

- linksventrikulärer Ausflusstrakt (B-Bild und Farbdoppler)

- rechtsventrikulärer Ausflusstrakt (B-Bild und Farbdoppler)

- Ductus arteriosus und Aortenbogen im Transversalschnitt (sog. Drei-Gefäßblick) oder im Längsschnitt (B-Bild und Farbdoppler)

- Einmündung der Pulmonalvenen in linkes Atrium (mittels Farbdoppler)

- das Vorliegen eines rechten Aortenbogens, einer A. lusoria und einer persistierenden linken oberen Hohlvene

Die Qualitätsanforderungen der DEGUM zur Durchführung der fetalen Echokardiographie bevorzugt für die 20.-22. SSW können bei entsprechender Indikation aber auch schon ab der 12. SSW angewandt werden (Chaoui et al. 2008, U. Gembruch et al. 2013).

Die sonografischen Einstellungen unterscheiden sich somit formal nicht von den Einstellungen, die im Rahmen der Echokardiographie im 2. Trimenon verwendet werden sollen.

Limitiert wird diese Anwendung dadurch, dass einige Einstellungen, wie die linksatriale Mündung der Pulmonalvenen, sehr niedrige erfolgreiche Darstellungsraten im ersten Trimester haben (Moon-Grady A 2012). Dabei steigt jedoch die Visualisierungsrate mit zunehmendem Gestationsalter (Haak MC 2002).

Nachteile der frühen fetalen Echokardiographie sind der nötige Zeitaufwand und der Bedarf an hochqualifizierten Untersuchern sowie hochauflösenden Ultraschallgeräten. Zudem besteht auch nach einer aufwendigen frühen Echokardiographie weiterhin die Möglichkeit eines sich entwickelnden Herzfehlers und somit kann auch bei einem unauffälligen Befund nicht auf eine spätere Echokardiographie ab der 18. SSW verzichtet werden (Neuman und Huhta 2006).

Laut DEGUM umfasst die Echokardiographie u.a. die komplette kardiale Bildgebung mittels konventionellen Ultraschalls (2D-Echokardiographie, M-Mode, pw- und cw-Doppler- 
Echokardiographie, farbkodierte Doppler-Echokardiographie) sowie mittels der modernen Anwendungsmöglichkeiten z.B. 3D/4D-Echokardiographie.

\subsubsection{Vorteil einer frühen Diagnosestellung}

Eine frühe Diagnosestellung gibt der Schwangeren die ausreichende Zeit, die eine intensive Auseinandersetzung mit der fetalen Problematik und eine interdisziplinäre Beratung möglich macht (Kaisenberg et al. 2016). Bei Hinweisen auf einen vorliegenden Herzfehler kann die Schwangerschaft schon zu einem frühen Zeitpunkt als Risikoschwangerschaft eingeordnet werden. Dementsprechend wird die Anzahl der Untersuchungen mit Intensivierung der Betreuung angepasst (Nicolaides 2011). Somit kann die Entwicklung des Herzfehlers bei früher Diagnosestellung besser beobachtet werden und in die Einschätzung der Prognose einfließen. Steht am Ende des Beratungsprozesses der Entschluss zum Schwangerschaftsabbruch, stellt der frühere Schwangerschaftsabbruch im Vergleich zu einem späteren Abbruch einen weniger risikoreichen und sowohl für die Angehörigen als auch für das medizinische Personal weniger belastend empfundenen Eingriff dar (Davies et al. 2005, Becker und Wegner 2006, Daugirdaite et al. 2015, Kaisenberg et al. 2016, Hutchinson et al. 2017). Vor allem in Fällen, in denen eine Lebensfähigkeit des Fetus bei jedoch stark eingeschränkter Lebensqualität zu erwarten ist, sollten Eltern so früh wie möglich die relevanten Informationen zur Verfügung zu stehen (Becker und Wegner 2006).

In mehreren großen Studien wurde belegt, dass durch die pränatale Diagnose des Herzfehlers, vor allem bei Feten mit schweren Herzfehlern wie TGA oder HLHS, eine Verbesserung des perinatalen Managements sowie die Senkung der Mortalität und Morbidität erreicht werden konnte (Tworetzky et al. 2001, Meyer-Wittkopf und Hofbeck 2003).

Zudem können immer früher Aussagen zu Entwicklungsstörungen des Herzens durch den Einsatz zunehmend hochwertiger Ultraschallgeräte sowie durch die steigende Erfahrung der Untersucherinnen und Untersucher getroffen werden.

In seltenen Fällen ist eine intrauterine Behandlung von Herzfehlern möglich. Jedoch ist aktuell bei mehr als 90 Prozent der komplexen angeborenen Herzfehler eine frühzeitige pränatale Detektion mit optimierter Schwangerschaftsüberwachung zur Vermeidung einer intrauterinen kardialen Dekompensation ausreichend. Dies wird auf die fest etablierten Behandlungskonzepte für die postnatale Behandlung zurückgeführt (Meyer-Wittkopf 2003).

Ein Beispiel für die Indikation zur Fetalchirurgie stellen die schweren Klappenstenosen wie Pulmonal- oder Aortenstenose dar. 
Unbehandelt führt die kritische Aortenklappenstenose (mit Flussumkehr in der Aorta) in $80 \%$ der Fälle zu einem HLHS. Als minimal-invasiver perkutaner transthorakaler Eingriff kann bei entsprechender Indikation die intrauterine aortale Ballonvalvuloplastie erfolgen, um ein weiteres Wachstum der Linksherzstrukturen zu sichern und so postnatal einen biventrikulären Repair zu ermöglichen (Gembruch et al. 2012).

Auch hier wird deutlich, dass frühzeitig entdeckte schwere Klappenstenosen oder Klappendysplasien einer engmaschigen Begleitung bedürfen und dadurch alle zur Verfügung stehenden Behandlungsoptionen genutzt werden können. 


\section{Ziele der Arbeit}

Die vorliegende Arbeit untersucht, ob durch die Beurteilung von drei kardialen Einstellungen - 4-Kammerblick, rechtsventrikuläre- und linksventrikuläre Ausflussbahn - im späten ersten Trimenon die Detektion angeborener Herzfehler möglich ist.

Die Reduzierung der frühen fetalen Echokardiographie auf 3 standardisierte kardiale Einstellungen soll es erlauben, eine orientierende, aber sichere Darstellung des fetalen Herzen zu erhalten. Damit müssen vor allem schwere und moderate Herzfehler sicher erkannt werden.

Zur Einordnung der Studie in die aktuelle Forschungslage erfolgen zudem eine Untersuchung des Kollektivs bezüglich der bekannten Risikofaktoren für angeborene Herzfehler, eine Aufschlüsselung zu Syndrom assoziierten Fehlbildungen und eine genaue Fallbeschreibung schwerer und moderater Herzfehler bezüglich Diagnose, Verlauf und Outcome der Kinder.

Folgende Hypothesen sollen dabei beantwortet werden:

1. Die in der Studie beschriebenen kardialen Einstellungen helfen bei der Aufdeckung schwerer oder moderater fetaler Herzfehler. Kardiale Minor-Anomalien können erst bei späteren Untersuchungen diagnostiziert werden.

2. Werden die in der Studie beschriebenen kardialen Einstellungen als unauffällig eingeschätzt, liegt mit hoher Wahrscheinlichkeit auch postnatal kein Herzfehler vor (hohe Spezifität).

3. Die frühe Diagnostik von fetalen Anomalien bringt aber auch die Gefahr einer vorschnellen Entscheidung zum Schwangerschaftsabbruch. Deshalb können die Ergebnisse dieser Studie als Basis für eine differenzierte und individuelle Beratung der Schwangeren genutzt werden, um vorschnelle Entscheidungen gegen die Fortsetzung der Schwangerschaft zu reduzieren.

4. Ein unauffälliges Ergebnis der kardialen Diagnostik im ersten Trimenon ist bei entsprechender Indikation kein Ersatz für eine Feindiagnostik im zweiten Trimenon. 


\section{Methodik}

\subsection{Auswahl Patientenkollektiv}

Einschluss in die Studie fanden schwangere Frauen, die im Zeitraum von 01.01.201331.12.2016 in einer Praxis für Pränataldiagnostik (DEGUM Stufe III) vorstellig wurden und sowohl eine frühe Feindiagnostik $(11+0-13+6 \mathrm{SSW})$ als auch eine Echokardiographie im zweiten Trimenon (ab 18. SSW) erhielten. Fälle, in denen ein Herzfehler diagnostiziert wurde und die Schwangerschaft durch einen Abbruch oder Abort vor einer weiteren Ultraschalluntersuchung endete, wurden ebenfalls eingeschlossen.

Unter diesen Kriterien fanden 1067 Schwangere mit insgesamt 1183 Feten Einschluss in die Studie. Das durchschnittliche Alter der eingeschlossenen Frauen betrug zum Zeitpunkt der ersten Untersuchung im Mittelwert 31,3 Jahre (Median 31 Jahre).

Diese Datenerhebung erfolgte retrospektiv und wurde um die Erhebung postnataler Daten ergänzt. Die postnatalen Daten wurden mittels Fragebogen (s. Anhang) oder der Auswertung des Krankenhausberichts ggf. mit paidopathologischer Untersuchung erhoben.

Alle Patientinnen wurden vor der Ultraschalluntersuchung aufgeklärt und gaben ihr schriftliches Einverständnis, dass die erhobenen Ergebnisse in Papierform sowie in elektronischer Form, entsprechend den gesetzlichen Vorgaben gespeichert und in pseudonymisierter Form für wissenschaftliche Zwecke oder für Zwecke der Qualitätssicherung genutzt/publiziert werden (Einverständniserklärung im Anhang). Mit den Fragebögen wurde eine Einverständniserklärung verschickt, in der die Patientinnen ihr Einverständnis zur Erhebung der für die Studien relevanten Daten in personenbezogener Form bei meinem behandelnden Arzt/Ärztin, sowie zur anonymisierten Weiterverarbeitung erteilten (Einverständniserklärung und Fragebogen im Anhang).

Bei der Ethikkommission des Universitätsklinikums Jena wurde die Studie angezeigt und unter der Nr. 2022-2512-Daten registriert. 


\subsection{Klassifikation der diagnostizierten Herzfehler}

Die Einteilung der pränatal diagnostizierten Herzfehler erfolgt nach der in der Einleitung genannten Klassifizierung der Herzfehler nach ihrer Komplexität in die erste Kategorie „schwere und moderate Herzfehler“ sowie in die zweite Kategorie „milde Herzfehler“ s. Kapitel 3.1.3.

Die Einteilung erfolgte nach der Diagnose aus der Echokardiographie im zweiten Trimenon. Feten, bei denen nur eine Herzuntersuchung im ersten Trimenon erfolgte (aufgrund von Abort oder durch Abbruch vorzeitig beendete Schwangerschaft), wurden nach der im Ersttrimester gestellten Diagnose klassifiziert.

Die pränatale Diagnose wurde dann mit den postnatalen Ergebnissen aus der Verlaufsbeobachtung verglichen.

Bei allen anderen Feten mit 2 Herzuntersuchungen (frühe fetale Herzuntersuchung und Echokardiographie ab der 18. SSW) erfolgte sowohl der Vergleich zwischen diesen Untersuchungen als auch, soweit vorhanden, der Vergleich mit postnataler Diagnose.

Diese Auswertung bezüglich der postnatalen Bestätigung erfolgte sowohl statistisch als auch als Einzelfallbeschreibung, da nicht bei allen Kindern mit Herzfehlern der postnatale echokardiographische Befund vorlag.

Postnatal diagnostizierte Herzfehler wurden separat betrachtet.

Für Kinder mit angeborenen schweren oder moderaten Herzfehlern wurde eine kasuistische Beschreibung bezüglich Procedere und Therapieplanung angelegt.

\subsection{Datenerhebung zum Patientenkollektiv}

Von den in der Studie eingeschlossenen Patienten wurden folgende Basis-Patientendaten aus den vorliegenden Befunden im Programm „View-Point Fetal Database Version 5.6.16“ entnommen:

Angaben zur Schwangeren:

- Alter zum Zeitpunkt der frühen Feindiagnostik

- BMI zum Zeitpunkt des Ersttrimesterscreenings (s.o.) in $\mathrm{kg} / \mathrm{m}^{2}$, Einteilung erfolgt nach WHO für $>20$ Jährige (World health organization 04/2020)

- relevante Grunderkrankungen nach Katalog A und B Mutterpass

- Medikamentenanamnese

Angaben zur Familienanamnese bezüglich Herzfehlern

- auffällige Familienanamnese (Verwandtschaftsgrad und spezifischer Herzfehler) 
Angaben zur Schwangerschaft

- Zeitpunkt der Untersuchung

- Einschätzung Untersuchungsbedingung (mit Angabe Untersuchungsmethode)

- genetische Untersuchung mit Ergebnis der genetischen Untersuchung

- Zahl der Feten, ggf. Chorionizität von Mehrlingsgravidität

\subsection{Sonografische Untersuchung}

Die sonografische Untersuchung der Feten erfolgte an zwei Ultraschallsystemen:

1. Voluson E 8 Expert GE Healthcare Systems transabdominal mit einer RAB4-8-D Konvexsonde oder transvaginal mit einer RIC 6-12-D Endokavitärsonde und

2. Voluson E 10 Expert GE Healthcare Systems transabdominal mit einer RM 6C Konvexsonde oder transvaginal mit einer RIC 6-12-D Endokavitärsonde entsprechend den Empfehlungen der DEGUM für die weiterführende pränatale Untersuchung im ersten Trimenon (Kaisenberg et al. 2016) durch eine Untersucherin der DEGUM Stufe 3.

\subsubsection{Erstes Trimenon}

Mittels Farbdoppler wurden drei kardiale Parameter dargestellt:

1. Der linke und rechte atrioventrikuläre Einstrom (bezeichnet als „Vierkammerblick“) mit folgenden Parametern:

○ Nachweisbarkeit beider atrioventrikulärer Flüsse

○ Konkordanz bezüglich der Größe beider atrioventrikulärer Flüsse (d.h. beider Herzhälften)

○ Qualität des Blutflusses (Nachweis von Turbulenzen/Regurgitationen)

2. Der linksventrikuläre Ausfluss (LVOT) und 3. der rechtsventrikuläre Ausfluss (RVOT) mit folgenden Parametern:

- Nachweis beider Ausflussbahnen

- Nachweis der Überkreuzung der Ausflussbahnen

○ Konkordanz der Größe beider Ausflussbahnen

○ Qualität des Blutflusses (Hinweis auf Turbulenzen/Regurgitationen/retrograde Flussmuster) 
Die Einschätzungen dieser kardialen Einstellungen wurden wie folgt klassifiziert:

- unauffällig oder

- auffällig:

○ kontrollbedürftig

○ pathologisch

○ nicht beurteilbar

Zudem erfolgte bei auffälligen Einstellungen die Erfassung einer Verdachtsdiagnose.

Zusätzlich wurden folgende Parameter sonografisch erfasst und ggf. daraus resultierend die Einordnung in die Perzentilen vorgenommen:

- die Nackentransparenz, Nackentransparenzperzentile

- Scheitel-Steißlänge mit Perzentileneinordnung (Zuordnung Gestationsalter)

- extrakardiale anatomische Auffälligkeiten

\subsubsection{Zweites Trimenon}

Zum zweiten Untersuchungszeitpunkt zwischen der 18+0 und 21+6 SSW erfolgte eine Echokardiographie gemäß den Qualitätsanforderungen der DEGUM (Chaoui et al. 2008).

Abweichungen vom Normalbefund und die entsprechende Diagnose wurden dokumentiert. Zusätzlich erfolgten die Erfassung des Geschlechts des Feten sowie der Perzentile des Schätzgewichts als Mittelwert aus den gemessenen Biometrien und die Dokumentation nicht kardialer anatomischer Auffälligkeiten.

\subsection{Prospektive Verlaufsbeobachtung}

Die Patienten ohne pränatal diagnostizierten fetalen Herzfehler wurden telefonisch oder schriftlich mittels Fragebogen (s. Anhang) zum Verlauf der Schwangerschaft bzw. der kindlichen Entwicklung befragt, wobei der Mutterpass und das Kinder-Untersuchungsheft (,U-Heft") als Quelle genutzt wurden.

Folgende Follow-up Parameter wurden dabei erhoben:

- Schwangerschaftsverlauf (Abort/Abbruch/Lebendgeburt/Totgeburt) mit Angabe der SSW

- Geburtsdatum Kind

- Wurde postnatal ein nicht bekannter Herzfehler diagnostiziert? 
- kardial bedingte Besonderheiten bei der Geburt (Vorstellung in Geburtsklinik/Intensivstationsaufenthalt)

Der Verlauf der Schwangerschaften, in denen pränatal ein fetaler Herzfehler gefunden wurde, wurde mittels eines Anomalierücklaufbogens bzw. paidopathologischem Befund oder Ergebnis der postnatalen Echokardiographie ausgewertet.

\subsection{Statistik}

Die statistische Auswertung der Daten erfolgte mit dem Programm SPSS (Version 26 und 27) für MAC OS.

Zur Auswertung der metrisch skalierten Variablen erfolgte die Berechnung des Mittelwerts mit Angabe der Standardabweichung, Angabe des Medians sowie der Angabe des Bereichs von minimalem Wert und maximalem Wert.

Bei nominalen Variablen wurde der Chi-Quadrat-Test angewendet. Falls die erwarteten Zellhäufigkeiten von einer oder mehr Zellen kleiner als 5 waren, wurde für die Ableitung des p-Werts der Exakte Test nach Fisher verwendet.

Der Mittelwertvergleich bei unabhängigen Stichproben erfolgte mit t-Test. Die Berechnung von Signifikanzen, Spezifität, PPW und NPW erfolgte mittels 4-Feldertafel (s. Anhang). Bei Signifikanzberechnungen wurde das Signifikanzniveau $\alpha$ auf $5 \%$ festgelegt. 


\section{Ergebnisse}

\subsection{Untersuchungskollektiv}

Das Patientenkollektiv umfasst insgesamt die Daten von 1067 Schwangeren, welche im Zeitraum von 01.01.2013-31.12.2016 in einer Praxis für Pränatalmedizin, vorstellig waren. Alle Untersuchungen wurden von einer DEGUM III zertifizierten Ärztin durchgeführt. Das Kollektiv besteht aus 1050 Einlingsschwangerschaften sowie 62 Zwillings- und 3 Drillingsschwangerschaften mit insgesamt 1183 Feten.

Bis auf 18 Fälle, in denen die Schwangerschaft vorzeitig endete, wurde bei allen Schwangeren sowohl ein Ersttrimesterscreening als auch eine Echokardiographie im zweiten Trimenon durchgeführt.

Das Ersttrimesterscreening erfolgte in der Woche 11+0 - 13+6 p.m., im Mittel 12+3 SSW, und die spätere Ultraschalluntersuchung mit Echokardiographie in Woche 18+0 - 21+6, im Mittel 21+3 SSW.

Aussagekräftige Befunde konnten im Ersttrimesterscreening bei 1012 Untersuchungen (85,6 $\%)$ transabdominal erreicht werden. 16 Untersuchungen (1,4\%) wurden transvaginal und 154 (13\%) kombiniert durchgeführt.

Bei 637 von 1183 Fällen (53,8 \%) wurde ein postnatales Outcome bzw. paidopathologischer Befund dokumentiert.

Im Falle eines pränatal diagnostizierten Herzfehlers lag die Dokumentationsrate des Outcomes höher. So konnte bei 39 der 51 Fälle (76,5 \%) mit Herzfehler (darunter 15 der 21 Fälle mit schwerem oder moderatem Herzfehler) postnatal eine Echokardiographie oder eine pathologische Untersuchung gesichert werden.

Der postnatale Nachbeobachtungszeitraum betrug in Durchschnitt 18 Monate.

\subsection{Prävalenz Herzfehler}

Die Prävalenz aller pränatal gefundenen Herzfehler lag im untersuchten Patientenkollektiv bei 4,3 \% (51 von 1183 Fällen). Davon waren 41,2 \% ( $n=21)$ schwere oder moderate Herzfehler mit einer Gesamtprävalenz von 1,8\% s. Abb. 17. 


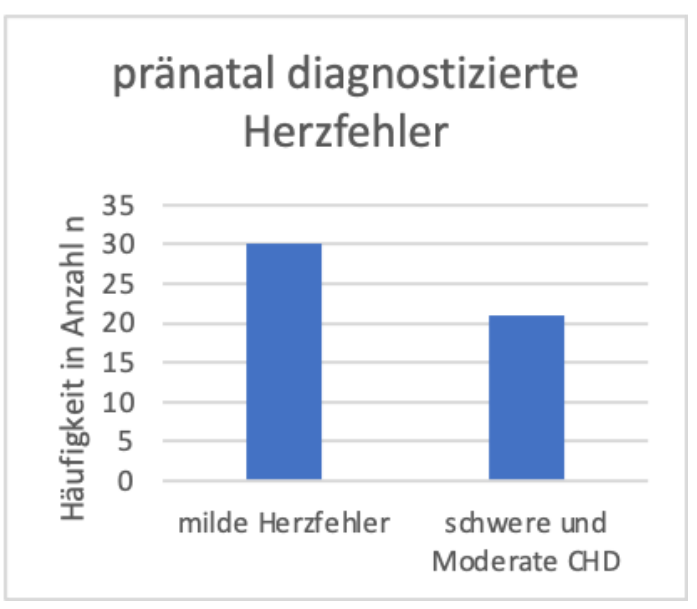

Abb. 17: Darstellung der 2 klassifizierten Herzfehler: schwere oder moderate Herzfehler $n=21$, milde Herzfehler $n=30$, insgesamt 51 Fälle

\subsubsection{Isolierte Herzfehler}

Isolierte Herzfehler lagen bei 30 von 51 Fällen (58,8 \%) vor. Milde Herzfehler machen hier einen Anteil von 83,3\% $(\mathrm{n}=25)$ aus. Der Anteil an isolierten Herzfehlern bei allen milden Herzfehlern beträgt demnach 83,3\%. Bei den 21 Feten mit schwerem oder moderatem Herzfehler lag die Rate an isolierten Herzfehlern mit insgesamt 28,6 \% deutlich niedriger s. Abb. 18.

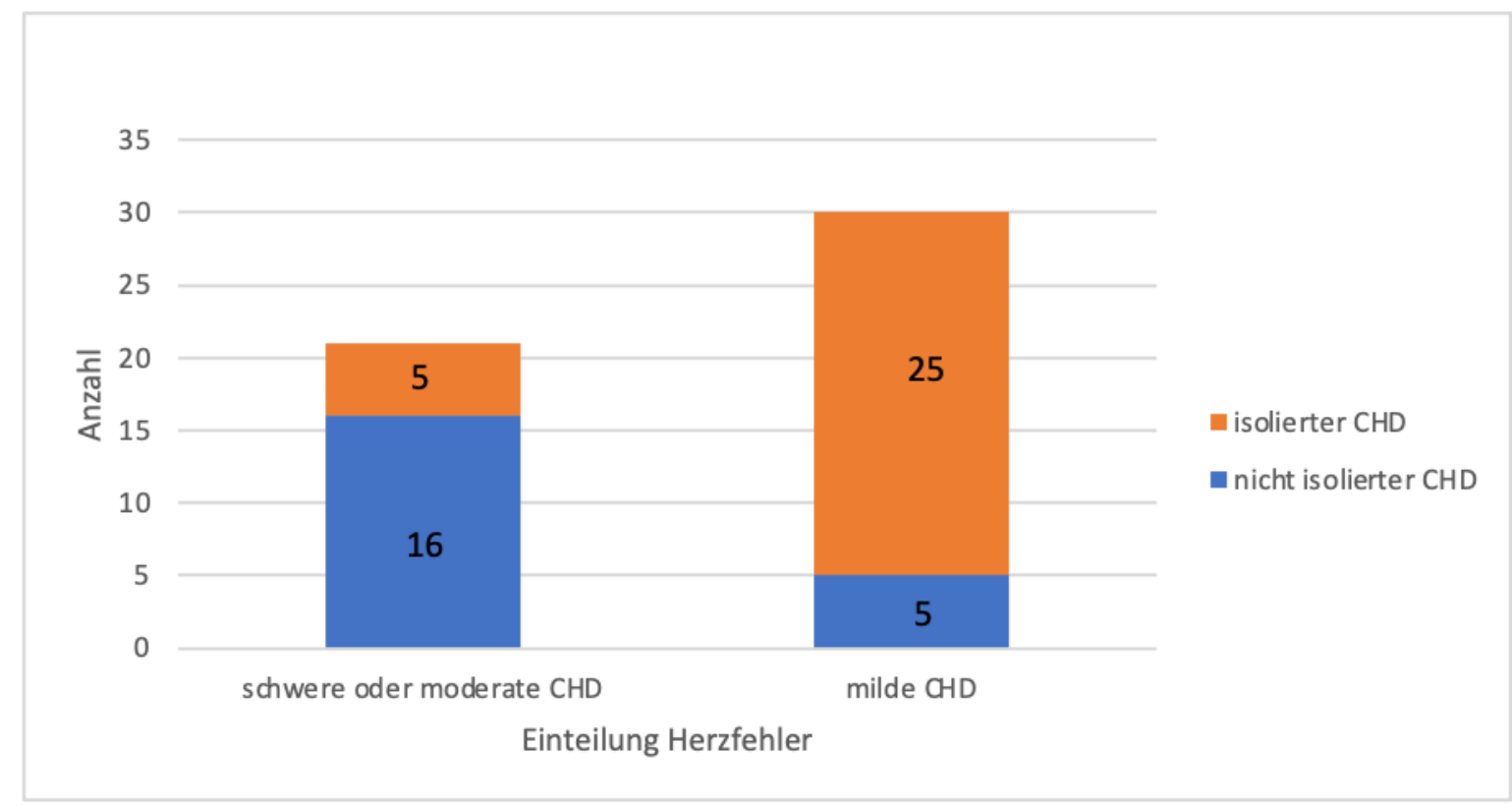

Abb. 18: Aufteilung aller pränatal gefundenen Herzfehler (CHD) nach isoliert oder nicht isoliert in die Schwere der vorliegenden Herzfehlerform 
Isolierte Herzfehler im ersten Trimenon:

20 der insgesamt 51 Fälle mit Herzfehlern wurden im ersten Trimenon als mindestens auffällig beschrieben. 5 der 20 Fälle (25\%) hatten hierbei isolierte Herzfehler.

Unter den 15 Fällen (75 \%) mit nicht isolierten Herzfehlern wurden 5 Fälle mit erhöhter NTP, 3 Fälle mit weiteren anatomischen Auffälligkeiten und 7 Fälle mit beidem gefunden.

Die mit schweren und moderaten Herzfehlern assoziierten Anomalien und genetischen Störungen sind in Tabelle 8 aufgeführt.

\subsubsection{Chromosomale Anomalien und syndromale Störungen}

Insgesamt wurde bei 138 von 1183 Feten eine genetische Diagnostik durchgeführt (Punktionsrate 11,7 \%). Von diesen 138 Feten hatten 110 Feten keinen Herzfehler (Punktionsrate bei Feten ohne Herzfehler: 9,7\%). Bei 28 Feten wurde ein Herzfehler diagnostiziert (Punktionsrate bei allen Feten mit Herzfehlern: 54,9 \%). In der Gruppe mit als schwer oder moderat kategorisiertem Herzfehler erfolgte bei 14 der 21 Fälle eine invasive pränatale Diagnostik (Punktionsrate 66,7 \%).

Auffällige Ergebnisse der genetischen Untersuchung lagen in der Gruppe ohne Herzfehler bei 4,5 \% der 110 Untersuchten und in der Gruppe mit Herzfehlern bei 25,5\%.

Somit besteht zwischen den Gruppen mit bzw. ohne Herzfehler ein signifikanter Unterschied bezüglich des Ergebnisses einer auffälligen genetischen Untersuchung ( $p=<0,001)$. Die Ergebnisse der genetischen Untersuchung sind in Tabelle 4 aufgeführt. 
Tab. 4: Ergebnisse genetischer Untersuchung

\begin{tabular}{|l|l|l|}
\hline Genetisches Ergebnis/Syndrom & Kategorie Herzfehler & Anzahl \\
\hline $\begin{array}{l}\text { Kurz-Rippen- } \\
\text { Polydaktyliesyndrom }\end{array}$ & Schwer/moderat & 1 \\
\hline Noonan-Syndrom & Schwer/moderat & 2 \\
\hline Trisomie 21 & Schwer/moderat & 4 \\
\hline Trisomie 21 & Leicht & 1 \\
\hline Trisomie 21 & Kein Herzfehler & 3 \\
\hline Trisomie 13 & Schwer/moderat & 1 \\
\hline Turner-Syndrom & Schwer/moderat & 1 \\
\hline Turner-Syndrom & Kein Herzfehler & 1 \\
\hline Holt-Oram-Syndrom & Schwer/moderat & 1 \\
\hline Di-George-Syndrom & Leicht & 1 \\
\hline Apert-Syndrom & Leicht & 1 \\
\hline Trisomie 20 & Kein Herzfehler & 1 \\
\hline
\end{tabular}

\subsection{Sensitivität und Spezifität der kardialen Einstellungen}

In 68 von 1183 Fällen (5,7 \%) wurde mindestens einer der 3 kardialen Parameter in der FFH (frühen fetalen Herzuntersuchung) als entweder „kontrollbedürftig/pathologisch/nicht beurteilbar“ eingeordnet. Davon wiesen 20 Fälle $(29,4 \%)$ einen Herzfehler (schwer/moderat/leicht) auf. Dies ergibt eine Sensitivität von 39,2 \% und eine Spezifität von $95,7 \%$.

In der Gruppe der schweren und moderaten Herzfehler $(n=21)$ ergibt sich eine Sensitivität von $90,4 \%$ und eine Spezifität von 95,7 \%. In dieser Population ist die Wahrscheinlichkeit, bei auffälligen kardialen Einstellungen in der FFH tatsächlich einen schweren oder moderaten Herzfehler zu haben, bei 28,3 \% (PPW). Bei unauffälligen kardialen Einstellungen in der FFH liegt die Wahrscheinlichkeit, keinen schweren oder moderaten Herzfehler zu haben, bei 99,8 $\%(\mathrm{NPW})$.

Grenzt man die Auffälligkeit in der FFH bei mindestens einer der drei Einstellungen auf die Kategorie „kontrollbedürftig oder pathologisch“ $(n=17)$ ein, so erhält man für die Frage nach moderaten oder schweren Herzfehlern eine Sensitivität von 80,9 \% und eine Spezifität von $100 \%$. Der PPW liegt hier bei $100 \%$ und der NPW bei 99,6\% s. Tabelle 5. 
Tab. 5: Screening Performance der kardialen Einstellungen

\begin{tabular}{|l|l|l|l|}
\hline Parameter & $\begin{array}{l}\text { 4 KB und } \\
\text { Ausflussbahnen } \\
\text { „auffällig“ bei } \\
\text { allen Herzfehlern }\end{array}$ & $\begin{array}{l}\text { 4KB und } \\
\text { Ausflussbahnen } \\
\text {,auffällig“ bei } \\
\text { schwerem oder } \\
\text { moderatem CHD }\end{array}$ & $\begin{array}{l}\mathbf{4} \text { KB und Ausflussbahnen } \\
\text { „pathologisch/ } \\
\text { kontrollbedürftig“ bei } \\
\text { schwerem oder moderatem } \\
\text { CHD }\end{array}$ \\
\hline Sensitivität \% & 39,2 & 90,4 & 80,9 \\
\hline Spezifität \% & 95,7 & 95,7 & 100 \\
\hline PPW \% & 29,4 & 27,9 & 100 \\
\hline NPW \% & 97,2 & 99,8 & 99,6 \\
\hline Prävalenz \% & 4,3 & 1,8 & 1,8 \\
\hline
\end{tabular}

$\mathrm{PPW}=$ positiv prädiktiver Wert, NPW = negativ prädiktiver Wert, $4 \mathrm{~KB}=4$-Kammerblick, auffällig $=$,pathologisch/kontrollbedürftig/nicht beurteilbar“, Prävalenz = Prävalenz der jeweils untersuchten Gruppe im Gesamtkollektiv

\subsection{Sonografische Einstellungen in der FFH}

\subsubsection{Einzelbetrachtung Feten mit Herzfehlern}

Unterteilt wird zunächst in Feten mit schweren oder moderaten Herzfehlern und Feten mit milden Herzfehlern.

Von den 21 Fällen mit schweren oder moderaten kardialen Anomalien wurden bei 19 Fällen (90,4 \%) im Ersttrimesterscreening mindestens eine der 3 kardialen Einstellungen als auffällig (,pathologisch/kontrollbedürftig/nicht beurteilbar“) beschrieben s. Abb. 19. 

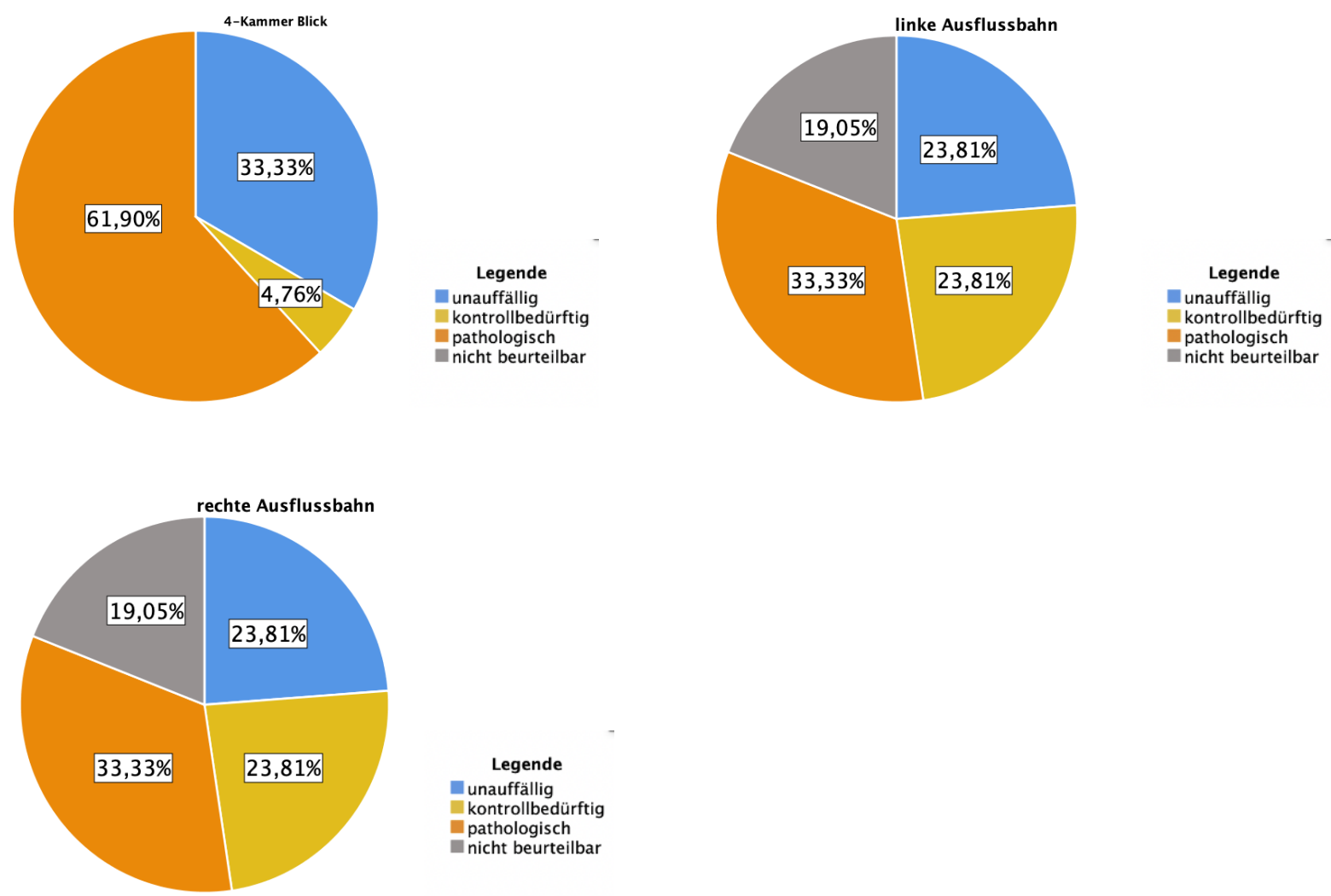

Abb. 19: Darstellung der kardialen Einstellung in der frühen FD bei Feten mit schweren oder moderaten Herzfehlern $(n=21)$

Für die 30 Feten mit leichten Herzfehlern stellt sich die Aufteilung wie folgt dar s. Abb.20:
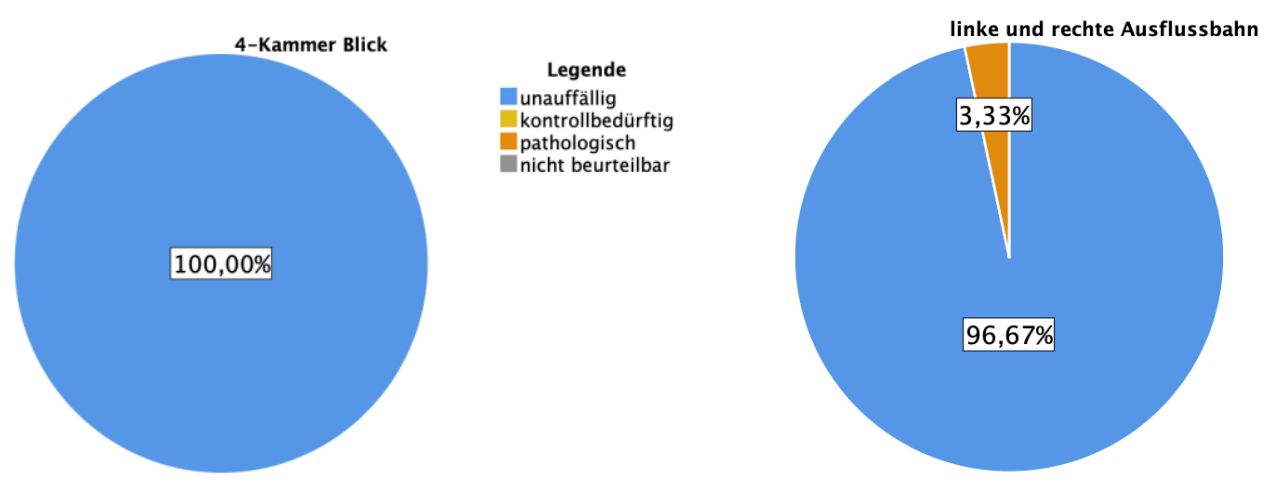

Legende

unauffällig kontrollbedürftig pathologisch nicht beurteilbar

Abb. 20: Darstellung der kardialen Einstellung in der frühen FD bei Feten mit leichten Herzfehlern, Anteil in Prozent $(\mathrm{n}=30)$ 


\subsubsection{Einzelbetrachtung der Einstellungen}

\section{Pathologisch und kontrollbedürftig}

In 17 der 1183 untersuchten Fälle $(1,4 \%)$ wurde mindestens eine der drei kardialen Einstellungen als ,pathologisch“ oder „kontrollbedürftig“ eingeschätzt. Davon hatten 100\% einen pränatal diagnostizierten Herzfehler s. Abb. 21.

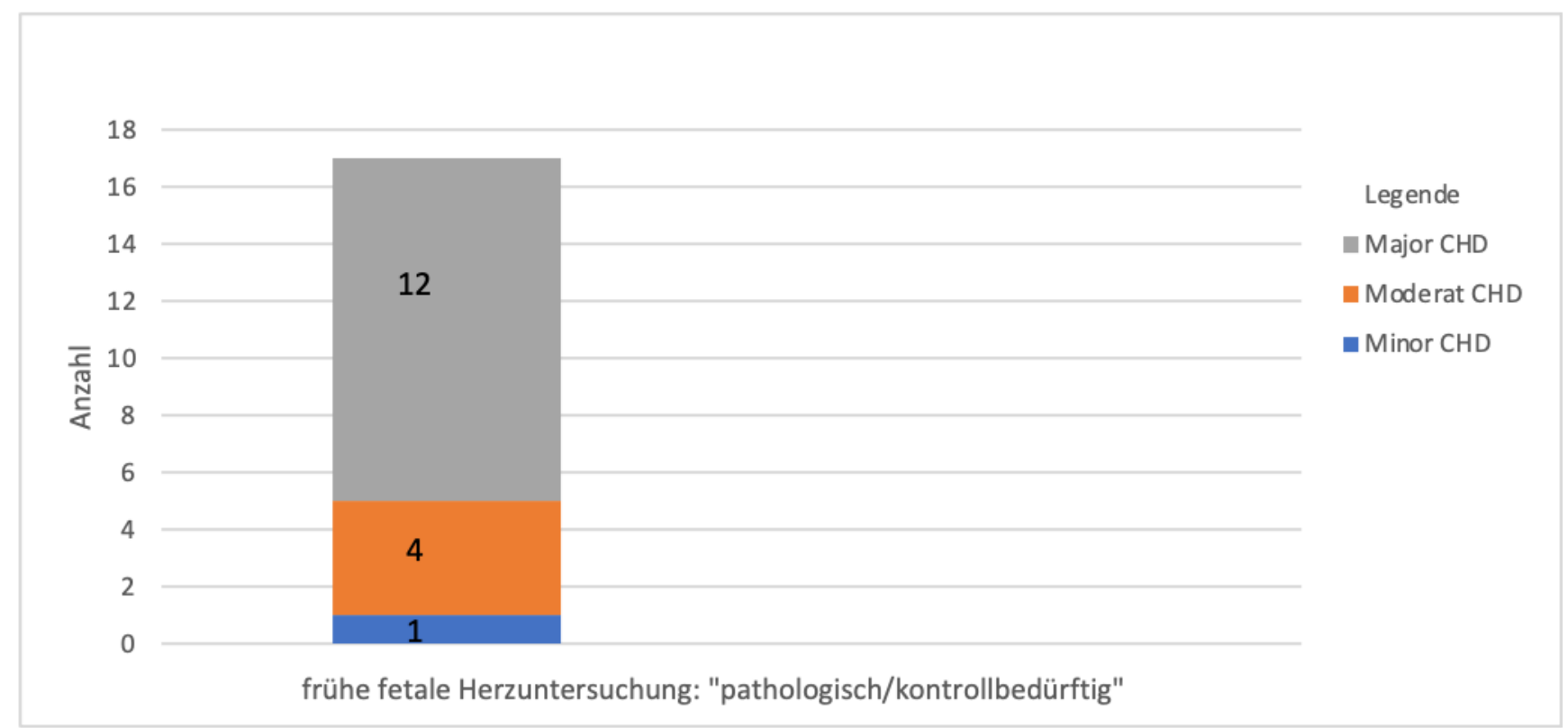

Abb. 21: Aufteilung der Fälle, in denen die kardialen Einstellungen in der frühen FD als „pathologisch“ oder „kontrollbedürftig“ beschrieben wurden, nach klassifizierten Herzfehlern, Anzahl in Fällen

$($ major $=$ schwer, minor $=$ mild $)$

\section{Nicht beurteilbar}

In 52 von 1183 untersuchten Fällen (4,4\%) wurde mindestens eine der drei kardialen Einstellungen als ,nicht beurteilbar“ eingeschätzt. Ein Herzfehler fand sich in dieser Gruppe bei 4 Fällen $(7,7 \%)$. Diese 4 Fälle wurden den schweren oder moderaten Herzfehlern zugeordnet. Die Untersuchungsbedingungen in dieser Gruppe waren in 30/52 Fällen (57,7 \%) eingeschränkt.

\section{Der 4 Kammerblick}

In der Bewertung des 4 Kammerblicks teilt sich die Beurteilung der insgesamt 25 als auffällig (,pathologisch/kontrollbedürftig/nicht beurteilbar“) beschriebenen Fälle wie folgt auf s. Abb. 22. 


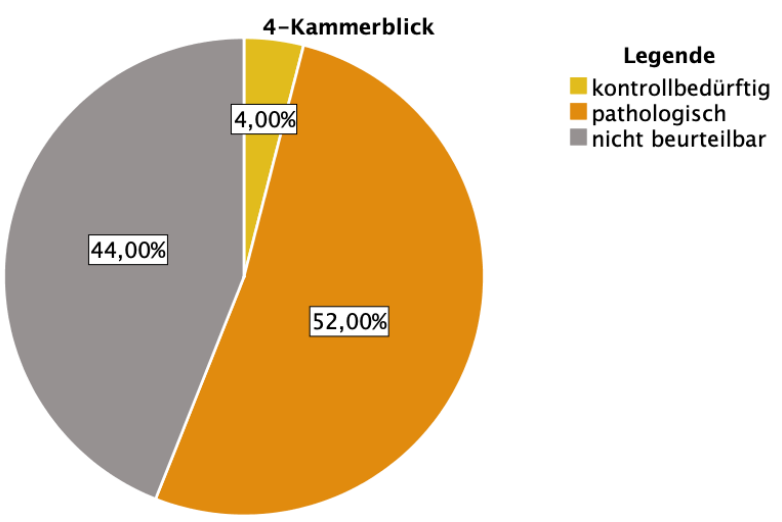

Abb. 22: Beurteilung 4-Kammerblick in der frühen fetalen Herzuntersuchung, kontrollbedürftig $n=1$, pathologisch $\mathrm{n}=13$ nicht beurteilbar $\mathrm{n}=11$.

In den 14 Fällen, bei denen der 4KB als ,,pathologisch“ oder „kontrollbedürftig“ eingeschätzt wurde, fand sich zu $100 \%$ ein schwerer oder moderater Herzfehler.

In den 11 Fällen, in denen der 4KB als ,nicht beurteilbar“ beschrieben wurde, lag in keinem Fall ein Herzfehler vor. Die Untersuchungsbedingungen waren in dieser Gruppe bei 7/11 Fällen $(63,3 \%)$ eingeschränkt.

\section{Die Ausflussbahnen LVOT und RVOT}

Auffälligkeiten (,kontrollbedürftig/pathologisch/nicht beurteilbar") im Bereich der Ausstrombahnen betrafen in allen Fällen immer beide Systeme - LVOT und RVOT s. Abb. 19. Insgesamt sind 65 Fälle mit auffälligen Ausstrombahnen beschrieben s. Abb. 23.

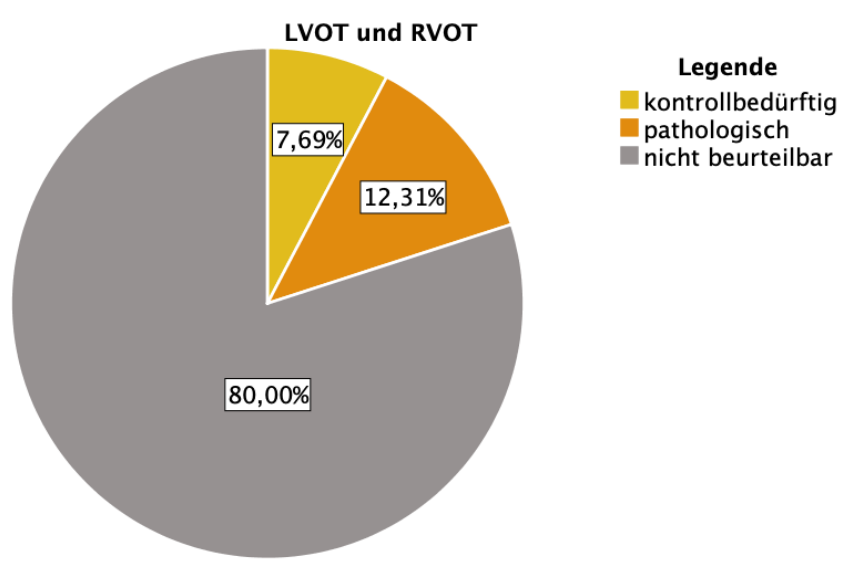

Abb. 23: Beurteilung LVOT und RVOT in der frühen fetalen Herzuntersuchung: kontrollbedürftig n= 5 , pathologisch $n=8$, nicht beurteilbar $n=52$.

Die 13 Fälle, deren Ausflussbahnen als ,pathologisch“ oder „kontrollbedürftig“ beschrieben wurden, hatten zu $100 \%$ einen Herzfehler. Darunter waren 12 Fälle mit schwerem oder moderatem Herzfehler und ein Fall mit einem leichten Herzfehler. 


\subsection{Untersuchungsbedingungen und Untersuchungsmethode}

Eingeschränkte Untersuchungsbedingungen fanden sich in 251 der $1183(21,2 \%)$ untersuchten Feten.

Angegebene Gründe dafür waren Adipositas, Retroflexio uteri, Voroperationen oder eine Vorderwandplazenta. Bei 220 der 251 Fälle (87 \%) war Adipositas allein oder in Kombination ursächlich für die eingeschränkten Bedingungen, mit einem durchschnittlichen BMI von 32,5 $\pm 5,0 \mathrm{~kg} / \mathrm{m}^{2}$ (Median: $31 \mathrm{~kg} / \mathrm{m}^{2}$, Bereich: 20,6-51,4 kg/m²).

In dem Kollektiv mit eingeschränkten Untersuchungsbedingungen wurde in 30 Fällen (12\%) eine der kardialen Einstellungen als „nicht beurteilbar“ beschrieben. In der Gruppe ohne eingeschränkte Untersuchungsbedingungen wurden 22 Fälle $(8,8 \%)$ als „,nicht beurteilbar“ eingeschätzt.

Somit hat die Untersuchungsbedingung einen signifikanten Einfluss auf die Bewertung der kardialen Parameter als ,nicht beurteilbar“ in der frühen FD $(\mathrm{p}=<0,001)$.

Die Untersuchungsmethode erfolgte transabdominal, transvaginal oder kombiniert s. Tab. 6.

Tab. 6: Anteil Untersuchungsmethode im Gesamtkollektiv, bei eingeschränkten USB und „nicht beurteilbaren" kardialen Einstellungen

\begin{tabular}{|l|l|l|l|}
\hline Kollektiv & TV/TA in \% & TA in \% & TV in \% \\
\hline Gesamtkollektiv (n= 1183) & 13 & 85,6 & 1,4 \\
\hline $\begin{array}{l}\text { Eingeschränkte Untersuchungsbedingungen (n= } \\
\text { 251) }\end{array}$ & 32,3 & 64,5 & 3,2 \\
\hline $\begin{array}{l}\text { „Nicht beurteilbare“ kardiale Einstellungen in } \\
\text { der frühen FD (n= 52) }\end{array}$ & 25 & 73,1 & 1,9 \\
\hline
\end{tabular}

$\mathrm{TA}=$ transabdominal, $\mathrm{TV}=$ transvaginal

\subsection{Risikofaktoren}

\subsubsection{Maternales Alter und maternale Adipositas}

Im Durschnitt betrug das Alter der Schwangeren zum Zeitpunkt des Ersttrimesterscreenings der 1132 Feten ohne Herzfehler 31,2 \pm 4,8 Jahre (Median: 31 Jahre, Bereich 16 - 46 Jahre). In der Gruppe mit Herzfehlern $(\mathrm{n}=51)$ lag der Mittelwert des Alters bei 31,7 \pm 5,2 Jahre (Median: 32 Jahre, Bereich 21 - 42 Jahre). Das Alter von Schwangeren mit und ohne fetalem Herzfehler unterscheidet sich nicht signifikant $(\mathrm{p}=0,30)$.

Der Anteil an über 35-jährigen Müttern lag im Gesamtkollektiv bei 34,7 \%, bei Feten ohne Herzfehler bei 37,3\% und in der Gruppe mit Herzfehlern bei 39,2\% $(p=0,612)$. 
Der mittlere BMI betrug in der Gruppe ohne Herzfehler 24,6 \pm 5,4 kg/m² (Median: 23,2 $\mathrm{kg} / \mathrm{m}^{2}$, Bereich $14-67 \mathrm{~kg} / \mathrm{m}^{2}$ ) und in der Gruppe mit Herzfehlern 25,5 $\pm 3,8 \mathrm{~kg} / \mathrm{m}^{2}$ (Median: $25 \mathrm{~kg} / \mathrm{m}^{2}$, Bereich $19-36 \mathrm{~kg} / \mathrm{m}^{2}$ ). Somit besteht kein Unterschied zwischen beiden Gruppen $(\mathrm{p}=0,124)$.

Der Anteil adipöser Schwangerer ab einem BMI von $30 \mathrm{~kg} / \mathrm{m}^{2}$ machte in der Gruppe ohne Herzfehler 14,3 \% aus. In der Gruppe mit Herzfehlern lag der Anteil bei 13,7 \%.

\subsubsection{Nackentransparenz}

In 81 von 1183 Fällen (6,8 \%) zeigte sich eine erhöhte Nackentransparenz ( $>95$. Perzentile). In 55,6\% der insgesamt 81 Fälle mit erhöhter Nackentransparenz wurde eine genetische Untersuchung durchgeführt, mit einem auffälligen genetischen Ergebnis bei $20 \%$.

Unter den 81 Feten mit erhöhter NTP hatten 12 Feten (14,8 \%) einen Herzfehler. Diese Fälle wurden alle den schweren oder moderaten Herzfehlern zugeordnet. Somit zeigten 12/21 Fälle mit schwerem oder moderatem Herzfehler eine erhöhte Nackentransparenz (52,4 \% bei zwei fehlenden Angaben). Bei $33 \%$ der Feten mit schwerem oder moderatem Herzfehler, jedoch ohne chromosomale Störung in der genetischen Untersuchung, lag die NTP über der 95. Perzentile.

Im Kollektiv ohne Herzfehler betrug die Nackentransparenz im Durchschnitt 1,79 \pm 0,69 mm (Median: 1,7 mm, Bereich: 0,8 - 11,8 mm).

Bei allen 51 Feten mit Herzfehlern lag der Mittelwert bei 2,5 \pm 1,8 mm (Median: 1,8 mm, Bereich 1,0 - 7,5 mm), bei Feten mit schwerem oder moderatem Herzfehler bei 3,92 $\pm 2,2$ mm (Median: 3,6 mm, Bereich: 1,1 - 7,5 mm).

Somit besteht im Mittelwert der Nackentransparenz ein signifikanter Unterschied zwischen Feten mit schwerem oder moderatem Herzfehler und Feten ohne Herzfehler $(\mathrm{p}=0,001) \mathrm{s}$. Abb. 24. Bei Feten mit leichten Herzfehlern zeigte sich im Mittelwert der Nackentransparenz kein signifikanter Unterschied zu Feten ohne Herzfehlern $(p=0,6)$. 


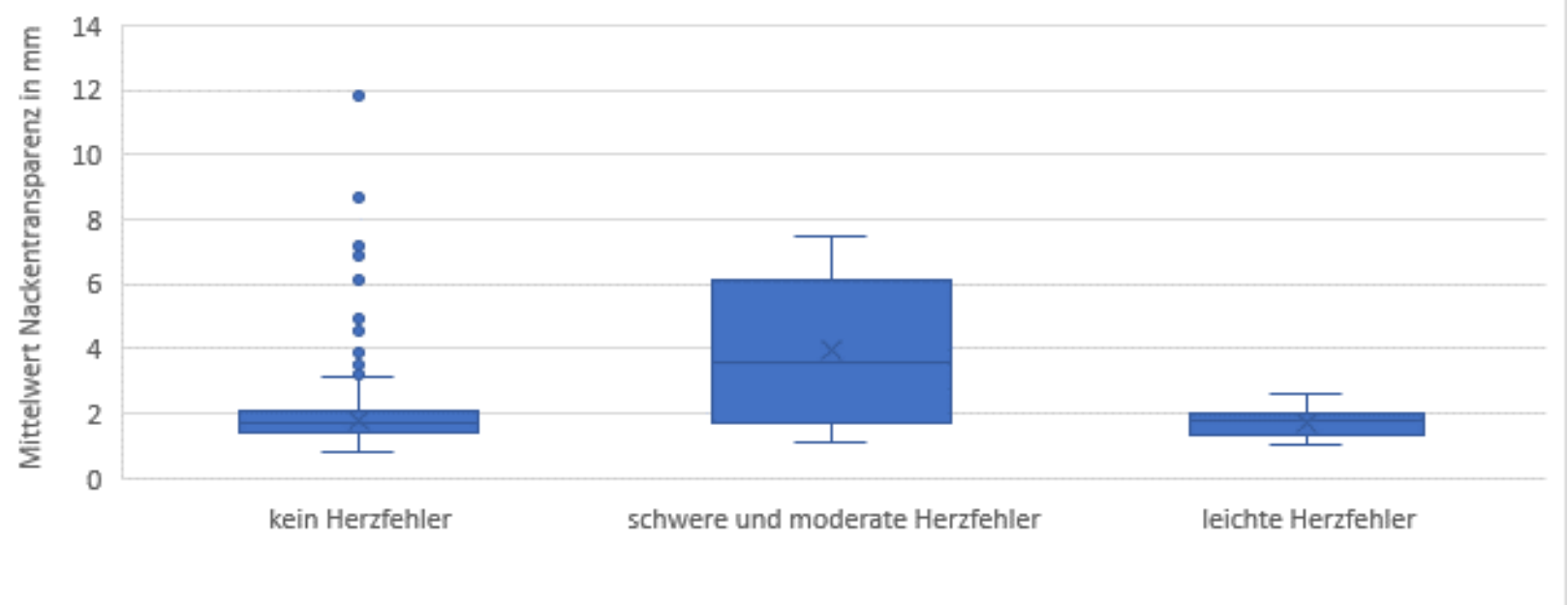

Abb. 24: Darstellung Nackentransparenz nach Quartilen, eingeteilt in Feten ohne Herzfehler und Feten mit schwerem oder moderatem Herzfehler sowie leichtem Herzfehler

\subsubsection{Weitere Risikofaktoren}

Die Auswertung weiterer Risikofaktoren für angeborene Herzfehler ergab keine signifikanten Unterschiede zwischen den Gruppen mit und ohne Herzfehler s. Tab 7.

Tab. 7: statistische Auswertung weiterer Risikofaktoren für angeborene Herzfehler

\begin{tabular}{|l|l|l|l|l|l|}
\hline Risikofaktor & $\begin{array}{l}\text { Gesamtkollek- } \\
\text { tiv (n=1183) }\end{array}$ & $\begin{array}{l}\text { G1: Mit } \\
\text { Herzfehler } \\
\text { (n=51) }\end{array}$ & $\begin{array}{l}\text { Schwere und } \\
\text { moderate } \\
\text { Herzfehler } \\
(\mathbf{n}=\mathbf{2 1})\end{array}$ & $\begin{array}{l}\text { G2: Ohne } \\
\text { Herzfehler } \\
\text { (n=1132) }\end{array}$ & $\begin{array}{l}\text { Statistische } \\
\text { Signifikanz }\end{array}$ \\
\hline $\begin{array}{l}\text { Auffällige } \\
\text { Familienanamnese } \\
\text { (Verwandtschaft 1. } \\
\text { Grades) }\end{array}$ & $4,7 \%$ & $\begin{array}{l}7,8 \% \text { in der } \\
\text { Verwandt- } \\
\text { schaft } 1 . \\
\text { Grades }\end{array}$ & $\begin{array}{l}9,5 \% \text { in der } \\
\text { Verwandt- } \\
\text { schaft 1. Grades }\end{array}$ & $\begin{array}{l}4,6 \% \text { in der } \\
\text { Verwandt- } \\
\text { schaft } 1 . \\
\text { Grades }\end{array}$ & $\mathrm{p}=0,29$ \\
\hline $\begin{array}{l}\text { Medikamentenein- } \\
\text { nahme }\end{array}$ & $31,7 \%$ & $35,3 \%$ & $38,1 \%$ & $31,5 \%$ & $\mathrm{p}=0,573$ \\
\hline Diabetes mellitus & $0,7 \%$ & $0 \%$ & $0 \%$ & $0,7 \%$ & $\mathrm{p}=1,0$ \\
\hline $\begin{array}{l}\text { Weitere mütterliche } \\
\text { Stoffwechselstörung }\end{array}$ & $17,8 \%$ & $13,7 \%$ & $4,8 \%$ & $18 \%$ & $\mathrm{p}=0,433$ \\
\hline $\begin{array}{l}\text { Anwendung assist. } \\
\text { Reproduktionstech- } \\
\text { nologie (IVF/ISCI) }\end{array}$ & $5,7 \%$ & $3,9 \%$ & $9,5 \%$ & $5,8 \%$ & $\mathrm{p}=1,0$ \\
\hline $\begin{array}{l}\text { Monochoriale } \\
\text { Geminigravidität }\end{array}$ & $3,0 \%$ & $2,0 \%$ & $4,8 \%$ & $3,1 \%$ & $\mathrm{p}=0,76$ \\
\hline
\end{tabular}


Unter den 1183 untersuchten Feten konnte von 1161 Feten das Geschlecht im 1. Trimenon ermittelt werden. Es fanden sich $52 \%$ männliche Feten und 47,8 \% weibliche Feten sowie 1 Fall $(0,08 \%)$ mit indifferentem Geschlecht.

Zwischen den Geschlechtern besteht kein signifikanter Unterschied bei der Frage nach einem Herzfehler $(p=0,103)$.

\subsection{Schwangerschaftsabbruch und Spontanaborte}

Im Kollektiv ohne Herzfehler wurden 0,4\% $(n=4)$ der Schwangerschaften aus medizinischer Indikation nach $\S 218 \mathrm{StGB}$ nach der 12. SSW p.c. durch einen Abbruch beendet. Gründe hierfür waren chromosomale Anomalien wie Trisomie 21, Embryoreduktion bei Mehrlingsschwangerschaft sowie andere anatomische Fehlbildungen.

Im Durchschnitt wurde der Abbruch hier in der 13+6 \pm 1,8 SSW (Median: 14 SSW, Bereich: 12 - 16 SSW) durchgeführt.

Insgesamt erfolgte bei 11 der 51 Feten (21.5\%) mit Herzfehler ein Schwangerschaftsabbruch. Bei 9 der 11 Feten (81,8\%) wurde eine weitere anatomische Auffälligkeit oder genetische Störung gefunden.

In der Gruppe mit leichten Herzfehlern wurden 2 Schwangerschaften in der 17+6 und 24+3 SSW beendet. Hier lagen in beiden Fällen syndromale Erkrankungen vor (Trisomie 21 und Di-George Syndrom).

In der Gruppe mit schweren oder moderaten Herzfehlern $(n=21)$ lag die Abbruchrate bei 43 $\% \quad(n=9)$. Davon wurden die Herzfehler der Feten bei 8 der 9 abgebrochenen Schwangerschaften im ersten Trimenon diagnostiziert. Der Abbruch erfolgte bei diesen 8, im ersten Trimenon diagnostizierten schweren oder moderaten Herzfehlern im Durchschnitt in der 16+5 \pm 5,01 SSW (Median: 16 SSW, Bereich: 12 - 29 SSW). Der durchschnittliche Zeitraum von der Diagnosestellung im ersten Trimenon bis zum Abbruch der Schwangerschaft betrug 30,8 \pm SD 20,6 Tage (Median 28 Tage, Bereich: 4 - 66 Tage).

In dieser Gruppe zeigten 6 der 9 Feten $(66,7 \%)$ weitere teils schwere anatomische Fehlbildungen wie generalisierten Hydrops oder Gastrochisis.

Bei den 2 Feten mit im zweiten Trimenon diagnostiziertem schweren Herzfehler wurde in einem Fall die Schwangerschaft in der 29. SSW aufgrund der infausten Prognose beendet. Die Zeit zwischen Diagnose und Abbruch betrug 46 Tage. Der zweite Fall endete in einem Spätabort in der 24. SSW. 
Spontanaborte kamen in der Gruppe ohne Herzfehler bei 3 Fällen (0,26 \%) vor, in der Gruppe mit schweren oder moderaten Herzfehlern ebenfalls bei 3 Fällen (14,3\%). Alle 3 Feten hatten schwere komplexe fetale Anomalien. Bei Feten mit leichten Herzfehlern lag die Abortrate bei $6,7 \%(n=2)$. 


\subsection{Fallbeschreibung: schwere und moderate Herzfehler}

Tab. 8: Fälle mit schweren oder moderaten Herzfehlern

\begin{tabular}{|c|c|c|c|c|c|c|c|c|c|}
\hline Fall & $4 \mathrm{~KB}$ & $\begin{array}{l}\text { LV } \\
\text { OT }\end{array}$ & $\begin{array}{l}\text { RV } \\
\text { OT }\end{array}$ & GA & Diagnose frühe FD & Diagnose im Verlauf & $\begin{array}{l}\text { Genetische } \\
\text { Untersuchung }\end{array}$ & $\begin{array}{l}\text { Diagnose } \\
\text { postnatal/postmortal }\end{array}$ & Outcome \\
\hline 1 & $P$ & $\mathrm{~K}$ & K & $12+4$ & $\begin{array}{l}\text { univentrikuläres Herz, Ausflussbahnen } \\
\text { kontrollbedürftig s. Abb. } 25\end{array}$ & $\begin{array}{l}18+6 \text { SSW: Linksherzhypoplasie mit } \\
\text { tubulärer Aortenhypoplasie, retrograde } \\
\text { Aortenperfusion }\end{array}$ & $\begin{array}{l}\text { Karyogramm und } \\
\text { Array unauffällig }\end{array}$ & Diagnose bestätigt & Abruptio $21+1 \mathrm{SSW}$ \\
\hline 2 & 0 & NB & NB & $12+6$ & kontrollbedürftige Ausflussbahnen & $\begin{array}{l}\text { 18+0 SSW: Dilatation re Ventrikel } \\
\text { und re Vorhof, } \\
\text { 28+4 SSW: Dilatation re Ventrikel, re } \\
\text { Vorhof, Myokardhypertrophie rechts }\end{array}$ & $\begin{array}{l}\text { Mutation im Exon } \\
9 \text { des TBX5 Gens } \\
\text { (Holt Oram } \\
\text { Syndrom) }\end{array}$ & $\begin{array}{l}\text { ISTA mit PDA, ASD II, } \\
\text { muskulärer VSD }\end{array}$ & $\begin{array}{l}\text { Primäre sectio } 38+4 \\
\text { SSW }\end{array}$ \\
\hline 3 & 0 & NB & NB & $12+2$ & kontrollbedürftiges Herz & $\begin{array}{l}\text { 22+1 SSW: rechtsventrikuläre } \\
\text { hypertrophe Kardiomyopathie und } \\
\text { Hypoplasie }\end{array}$ & - & $\begin{array}{l}\text { Echo postnatal: kein } \\
\text { Anhalt für } \\
\text { Kardiomyopathie, kleiner } \\
\text { ASD II, LSVC, PFO und } \\
\text { PDA altersentsprechend } \\
\text { normal }\end{array}$ & $\begin{array}{l}\text { Spontangeburt } 40+5 \\
\text { SSW }\end{array}$ \\
\hline 4 & $\mathrm{P}$ & 0 & 0 & $11+2$ & $\begin{array}{l}\text { Univentrikuläres Herz } \\
+ \text { Hygroma colli }+ \text { NT }>95 \text {. Perzentile } \\
\text { s. Abb. } 26\end{array}$ & $\begin{array}{l}\text { 15+4. SSW: V.a. Entwicklung einer } \\
\text { Linksherzhypoplasie, } \\
\text { Mitralklappendysplasie + massiver } \\
\text { Hydrops fetalis, Hygroma colli }\end{array}$ & $\begin{array}{l}\text { PTPN11 Mutation } \\
\text { (Noonan } \\
\text { Syndrom) }\end{array}$ & $\begin{array}{l}\text { HLHS, Aortenatresie, } \\
\text { ASD I, Hygroma colli, } \\
\text { Hepatomegalie bei } \\
\text { erhöhter kardialer } \\
\text { Belastung }\end{array}$ & Abruptio $16+4 \mathrm{SSW}$ \\
\hline 5 & 0 & $\mathrm{P}$ & $\mathrm{P}$ & $12+0$ & $\begin{array}{l}\text { VSD, (große Gefäße unklar) + } \\
\text { generalisierter Hydrops fetalis }+ \text { NT }>95 \text {. }\end{array}$ & $\begin{array}{l}\text { 21+2 SSW: VSD und Verdacht auf } \\
\text { TAC + Nasenbeinhypoplasie }+\end{array}$ & $47 \mathrm{XX}+21$ & Diagnose bestätigt & Sectio $33+3$ SSW \\
\hline
\end{tabular}




\begin{tabular}{|c|c|c|c|c|c|c|c|c|c|}
\hline & & & & & Perzentile & manifeste IUWR & & & \\
\hline 6 & $\mathrm{P}$ & K & $\mathrm{K}$ & $11+2$ & Vd.a. Vitium cordis + NT $>95$. Perzentile & $\begin{array}{l}\text { 16+1 SSW univentrikuläres Herz + } \\
\text { Hydrops fetalis }\end{array}$ & $\begin{array}{l}\text { PTPN11 Mutation } \\
\text { (Noonan } \\
\text { Syndrom) }\end{array}$ & $\begin{array}{l}\text { Keine Paidopathologische } \\
\text { Untersuchung }\end{array}$ & Abruptio $18+4$ SSW \\
\hline 7 & 0 & K & K & $12+2$ & $\begin{array}{l}\text { Vitium cordis: zwei Herzhälften } \\
\text { darstellbar, im Ausfluss momentan nur ein } \\
\text { Gefäß darstellbar }\end{array}$ & $\begin{array}{l}\text { 23+2 SSW: V.a. DORV mit TGA- } \\
\text { Stellung der großen Gefäße, zusätzlich } \\
\text { VSD }\end{array}$ & - & $\begin{array}{l}\text { Postnatal TOF, Korrektur } \\
\text { OP mit } 6 \text { Monaten }\end{array}$ & $\begin{array}{l}\text { Sectio bei } \\
\text { Querlage38+4 SSW }\end{array}$ \\
\hline 8 & $\mathrm{P}$ & $\mathrm{P}$ & $\mathrm{P}$ & $13+5$ & $\begin{array}{l}\text { Univentrikuläres Herz } \\
\text { mit Hypoplasie des Truncus pulmonalis s. } \\
\text { Abb. } 27\end{array}$ & $\begin{array}{l}\text { 21+4 SSW: Mitralklappendysplasie, } \\
\text { univentrikuläres Herz DD DILV } \\
\text { über den eine Perfusion des LV } \\
\text { erfolgt. Große Gefäße unauffällig }\end{array}$ & - & $\begin{array}{l}\text { DILV, } \\
\text { Mitralklappenstenose, } \\
\text { PFO, Pulmonalstenose }\end{array}$ & $\begin{array}{l}\text { Sectio bei kardialer } \\
\text { Erkrankung } 31+5 \\
\text { SSW, Korrektur-OP } \\
\text { am 18. Lebenstag }\end{array}$ \\
\hline 9 & 0 & K & K & $13+2$ & $\begin{array}{l}\text { kardiale Ausflussbahnen kontrollbedürftig } \\
\text { s. Abb. } 28\end{array}$ & 20+3 SSW: d-TGA & $\begin{array}{l}\text { Unauffälliger } \\
\text { Karyotyp }\end{array}$ & Diagnose bestätigt & $\begin{array}{l}\text { sectio bei kardialer } \\
\text { Erkrankung } 38+0 \\
\text { SSW }\end{array}$ \\
\hline 10 & $\mathrm{P}$ & $\mathrm{P}$ & $\mathrm{P}$ & $13+3$ & $\begin{array}{l}\text { V.a. Vitium cordis: re Ventrikel geringer } \\
\text { perfundiert, retrograde Perfusion des } \\
\text { Truncus pulmon. + laterale Halszysten, } \\
\text { Nackenödem, Nasenbeinhypoplasie, NT } \\
>95 \text {. Perzentile s. Abb. } 29\end{array}$ & - & $\begin{array}{l}\text { Unauffälliger } \\
\text { Karyotyp }\end{array}$ & - & $\begin{array}{l}\text { missed abortion bei } \\
\text { generalisiertem } \\
\text { Hydrops fetalis } 16+5 \\
\text { SSW }\end{array}$ \\
\hline 11 & 0 & 0 & 0 & $13+2$ & Kein auffälliger Befund & $\begin{array}{l}\text { 22+3 SSW: V.a. dilatative } \\
\text { Kardiomyopathie beider Ventrikel, } \\
\text { eingeschränkte kardiale Kontraktilität, } \\
\text { Trikuspidalklappeninsuffizienz }\end{array}$ & - & $\begin{array}{l}\text { Obduktion: } \\
\text { Endokardfibroelastose } \\
\text { Herzdilatation und } \\
\text { Hypertrophie }\end{array}$ & $\begin{array}{l}\text { Spontangeburt nach } \\
\text { Geburtseinleitung } \\
\text { wegen infauster } \\
\text { Prognose } 28+6 \mathrm{SSW} \text {, } \\
\text { Kind nach } 45 \\
\text { Minuten verstorben }\end{array}$ \\
\hline 12 & $\mathrm{P}$ & $\mathrm{P}$ & $\mathrm{P}$ & $13+3$ & $\begin{array}{l}\text { Body stalk Anomalie } \\
\text { (Amnionruptursequenz) mit Ektopia cordis }\end{array}$ & - & - & Postnatal bestätigt & Totgeburt 33+5 SSW \\
\hline
\end{tabular}




\begin{tabular}{|c|c|c|c|c|c|c|c|c|c|}
\hline & & & & & + Anencephalie & & & & \\
\hline 13 & $P$ & NB & NB & $11+5$ & $\begin{array}{l}\text { univentrikuläres Herz }+ \text { Hirnzysten }+ \text { NT } \\
>95 . \text { Perzentile }\end{array}$ & - & $47 \mathrm{XY}+13$ & $\begin{array}{l}\text { Herz in 13. SSW nicht } \\
\text { beurteilbar }\end{array}$ & $\begin{array}{l}\text { Missed-abortion } 12+6 \\
\text { SSW }\end{array}$ \\
\hline 14 & $\mathrm{P}$ & $\mathrm{P}$ & $\mathrm{P}$ & $13+0$ & $\begin{array}{l}\text { Linksherzhypoplasie, retrograde } \\
\text { Aortenperfusion }+ \text { Skelettdysplasie } \\
\text { s. Abb. } 30\end{array}$ & $\begin{array}{l}\text { 14+6 SSW: Hypoplastisches } \\
\text { Linksherz, retrograde Aortenperfusion }\end{array}$ & $\begin{array}{l}\text { DYNC2H1 Gen } \\
\text { Mutation } \\
\text { (Kurzripp- } \\
\text { Polydaktylie- } \\
\text { Syndrom) }\end{array}$ & $\begin{array}{l}\text { Hypoplastisches } \\
\text { Linksherz mit } \\
\text { Kurzrippen- } \\
\text { Polydaktyliesyndrom }\end{array}$ & Abruptio $15+3 \mathrm{SSW}$ \\
\hline 15 & $\mathrm{P}$ & $\mathrm{P}$ & $\mathrm{P}$ & & $\begin{array}{l}\text { Bopdy stalk Anomalie } \\
\text { (Amnionruptursequenz) mit Ektopia cordis } \\
+ \text { Leber und Darm extraabdominal + NT } \\
>95 . \text { Perzentile }\end{array}$ & - & - & $\begin{array}{l}\text { Abortmaterial mit } \\
\text { Hinweis auf multipel } \\
\text { fehlgebildeten Embryo }\end{array}$ & $\begin{array}{l}\text { Missed abortion } 11+5 \\
\text { SSW }\end{array}$ \\
\hline 16 & $\mathrm{P}$ & $\mathrm{P}$ & $\mathrm{P}$ & $11+2$ & $\begin{array}{l}\text { Univentrikuläres Herz + Hydrops fetalis }+ \\
\text { NT }>95 \text {. Perzentile s. Abb. } 31\end{array}$ & - & $45 \mathrm{XO}$ & $\begin{array}{l}\text { Keine paidopathologische } \\
\text { Untersuchung }\end{array}$ & Abruptio $12+6 \mathrm{SSW}$ \\
\hline 17 & 0 & 0 & 0 & $12+5$ & Unauffällige Untersuchung & $\begin{array}{l}\text { 22+4 SSW: muskulärer VSD, } \\
\text { dilatative Kardiomegalie + schwere } \\
\text { IUWR + Hydrops fetalis bei } \\
\text { Zottenreifungsstörung }\end{array}$ & - & $\begin{array}{l}\text { Keine paidopathologische } \\
\text { Untersuchung }\end{array}$ & $\begin{array}{l}\text { Spätabort bei } \\
\text { Blasensprung } 23+6 \\
\text { SSW }\end{array}$ \\
\hline 18 & $\mathrm{~K}$ & $\mathrm{~K}$ & $\mathrm{~K}$ & $13+1$ & $\begin{array}{l}\text { V.a. AVSD + Nasenknochenhypoplasie, } \\
\text { NT }>95 . \text { Perzentile s. Abb. } 32\end{array}$ & - & $47 \mathrm{XY}+21$ & $\begin{array}{l}\text { Keine paidopathologische } \\
\text { Untersuchung }\end{array}$ & Abruptio $15+5 \mathrm{SSW}$ \\
\hline 19 & $P$ & 0 & 0 & $12+3$ & Kompletter AVSD + NT $>95$. Perzentile & $\begin{array}{l}\text { 20+2 SSW: kompletter AVSD + } \\
\text { Nasenknochenhypoplasie + borderline } \\
\text { Ventrikulomegalie }\end{array}$ & $\begin{array}{l}\text { Pränatal nicht } \\
\text { gewünscht }\end{array}$ & $\begin{array}{l}\text { Postnatal bestätigt } \\
\text { Trisomie } 21 \text { und AVSD }\end{array}$ & $\begin{array}{l}\text { geplante sectio bei } \\
\text { BEL } 38+2 \mathrm{SSW}\end{array}$ \\
\hline 20 & $P$ & 0 & 0 & $12+6$ & $\begin{array}{l}\text { AVSD, Trikuspidalklappeninsuffizienz }+ \\
\text { NT }>95 \text {. Perzentile }\end{array}$ & $\begin{array}{l}\text { 14+4 SSW: AVSD, } \\
\text { Trikuspidalklappeninsuffizienz + } \\
\text { Nackenödem + bilaterale Halszysten }\end{array}$ & $47 \mathrm{XY}+21$ & $\begin{array}{l}\text { paidopathologischer } \\
\text { Befund: AVSD bestätigt }\end{array}$ & Abruptio $15+5 \mathrm{SSW}$ \\
\hline 21 & $\mathrm{P}$ & NB & NB & $12+6$ & $\begin{array}{l}\text { VSD mit bidirektionalem Shunt }+ \text { NT }>95 \text {. } \\
\text { Perzentile s. Abb. } 33\end{array}$ & - & $47 \mathrm{XX}+21$ & - & Abruptio $12+6 \mathrm{SSW}$ \\
\hline
\end{tabular}




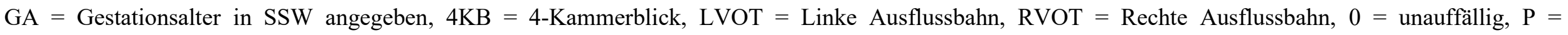
pathologisch, $\mathrm{K}=$ kontrollbedürftig, $\mathrm{NB}=$ nicht beurteilbar

\section{Bilddokumentation}

a)

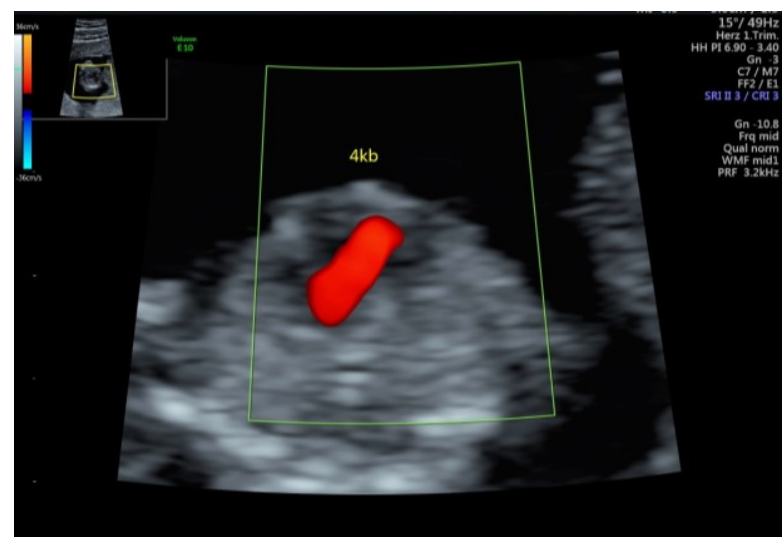

b)

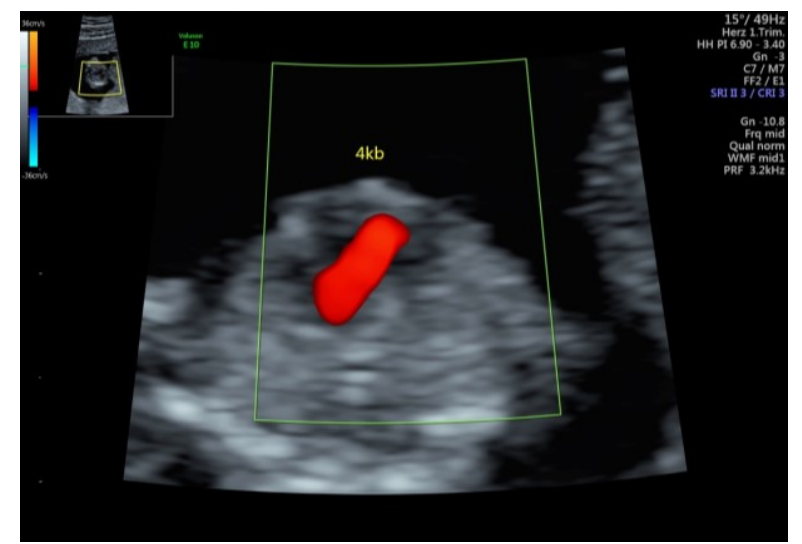

Abb. 25: Fall 1: univentrikuläres Herz a) 4KB: „pathologisch“, b) RVOT: „,kontrollbedürftig“ 

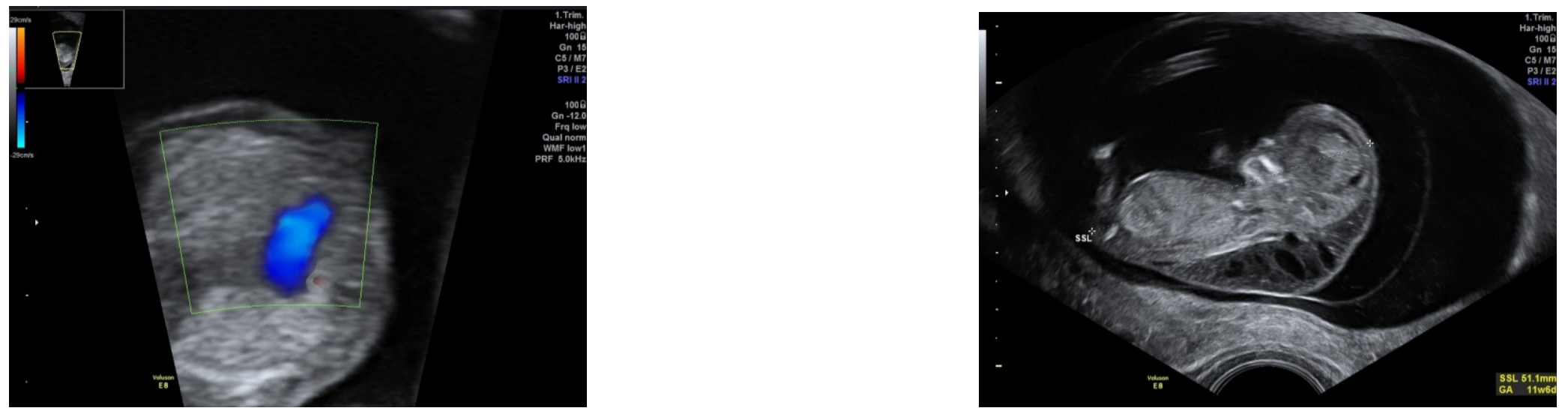

Abb. 26: Fall 4: univentrikuläres Herz, a) 4KB, b) Hygroma colli
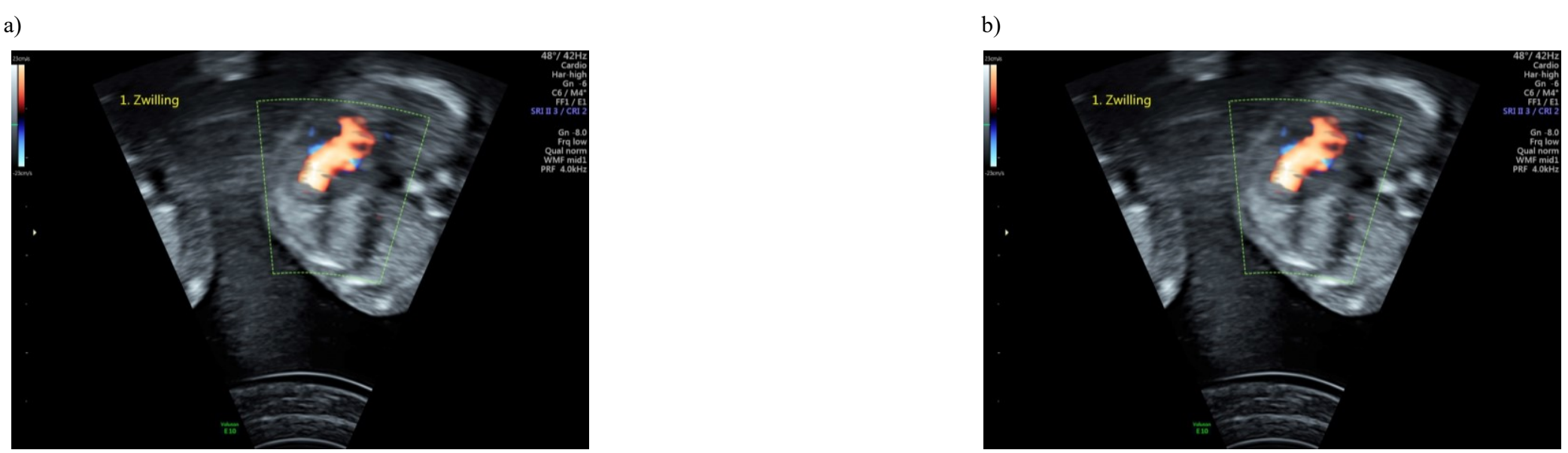

Abb. 27: Fall 8: univentrikuläres Herz, a) 4KB, b) Kaliberdifferenz der großen Gefäße im Dreigefäßblick 


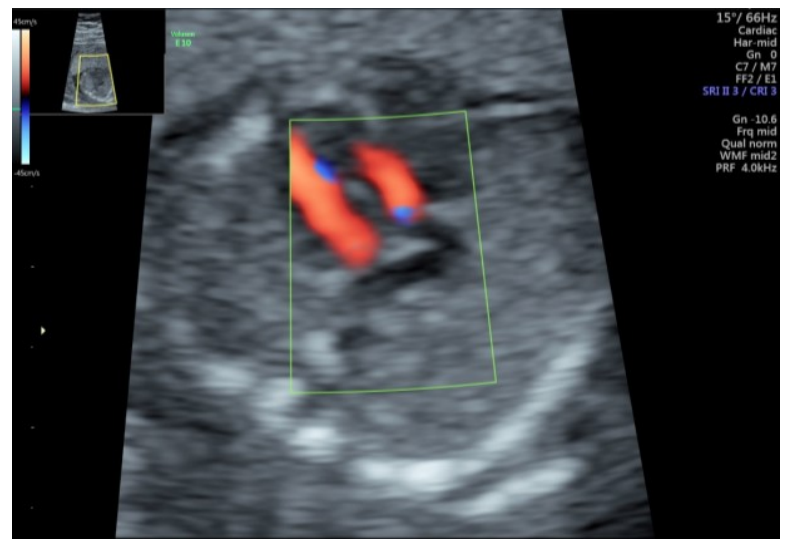

Abb. 28: Fall 9: d-TGA a) 4KB, b) Parallelverlauf der großen Gefäße bei d-TGA

a)

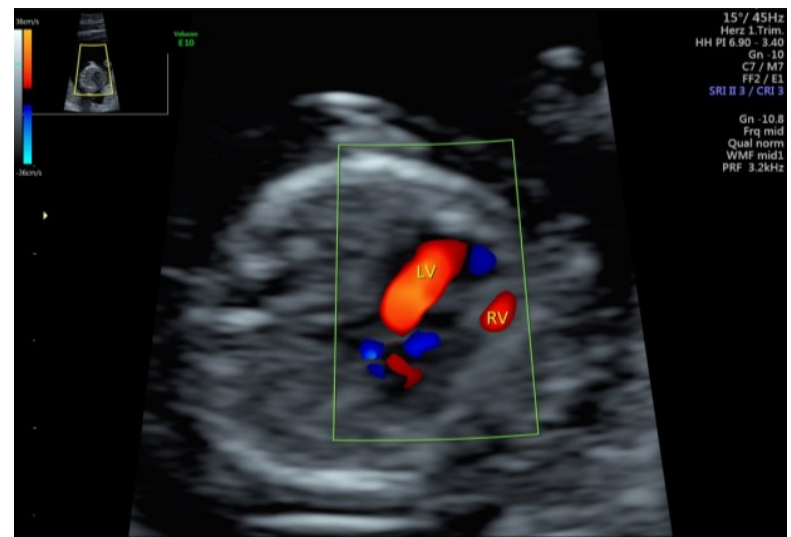

Abb. 29: Fall 10: a) 4KB, b) LVOT mit retrograder Perfusion des DAB

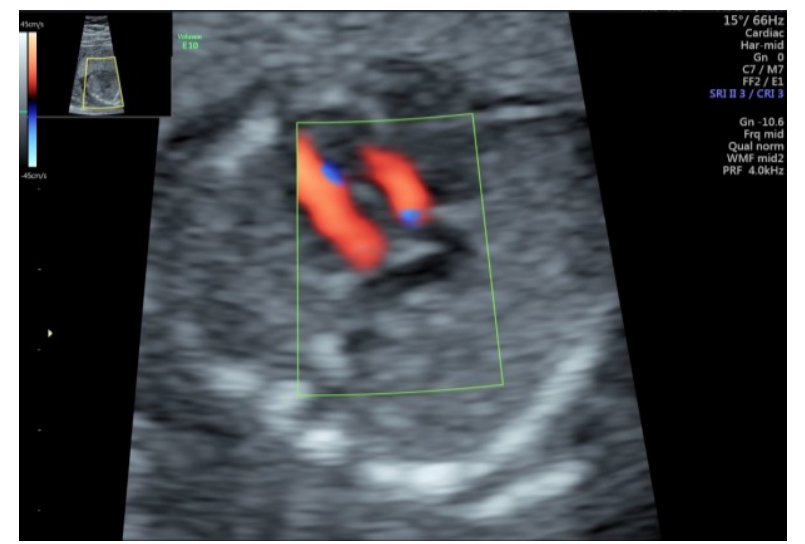

b)

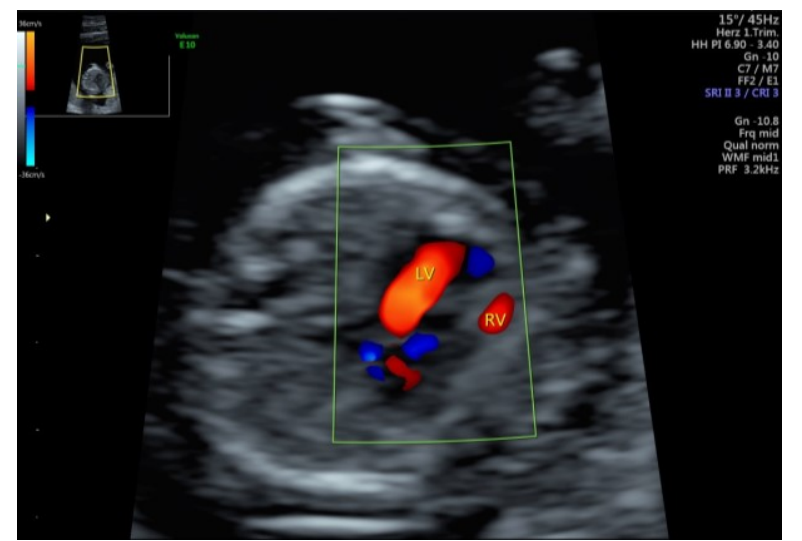




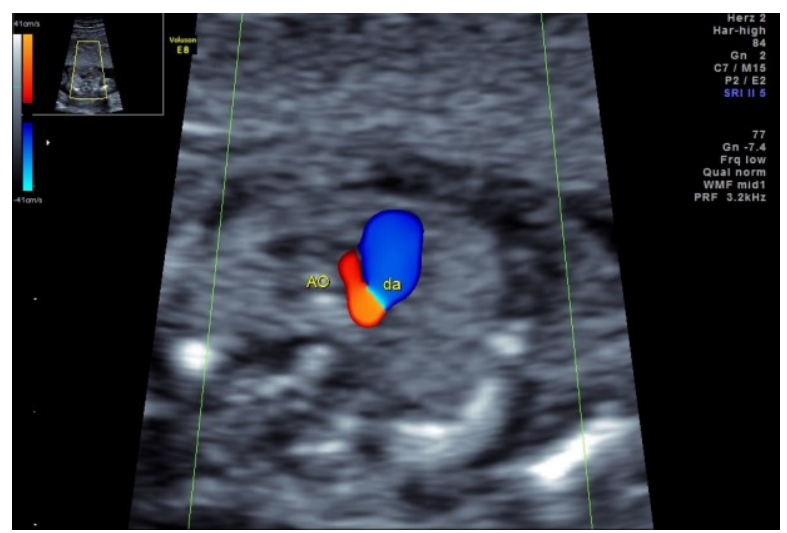

Abb. 30: Fall 14: Linksherzhypoplasie: RVOT mit retrograder Aortenperfusion

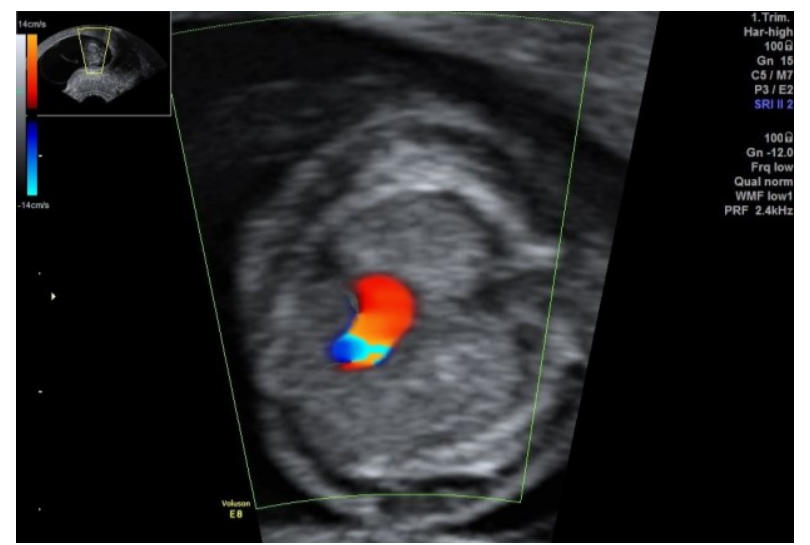

Abb. 31: Fall 16: Univentrikuläres Herz, 4KB 
a)

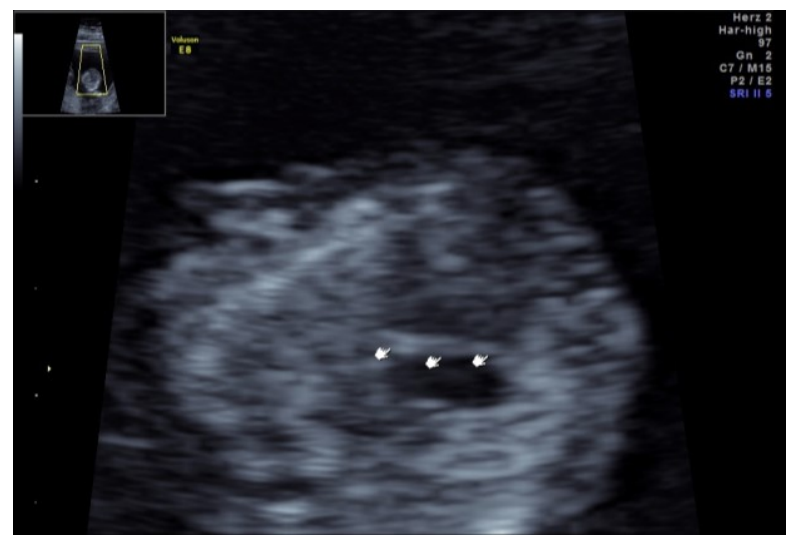

Abb. 32: Fall 18: AVSD, a) 4KB B-Bild: linearer AV-Klappenansatz, b) 4KB Farbdoppler: AVSD

a)

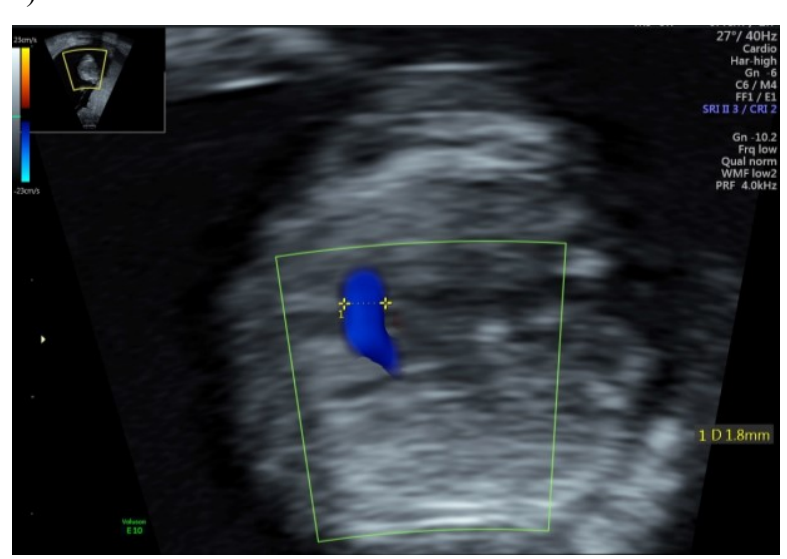

Abb. 33: Fall 21 VSD mit bidirektionalem Shunt, a) und b) 4KB b)

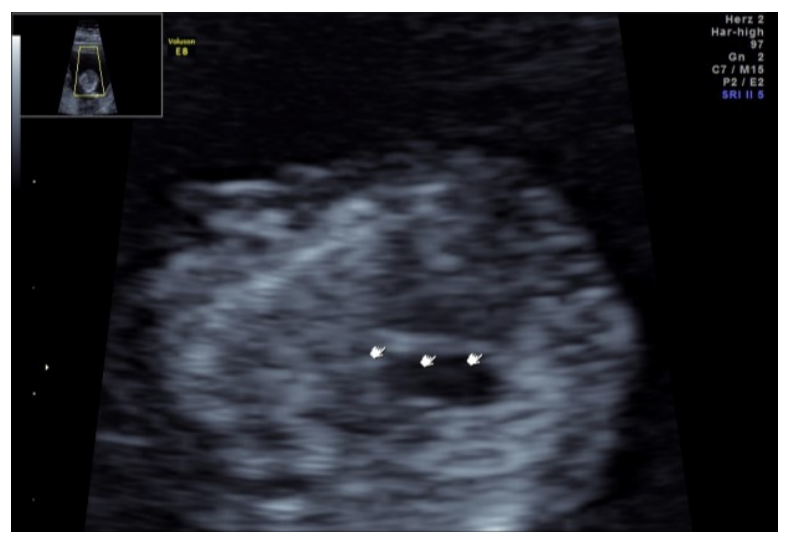

b)

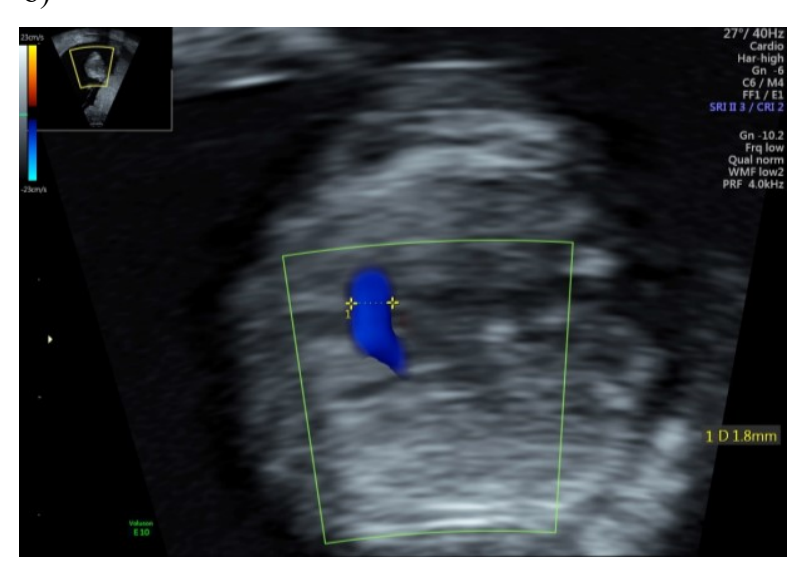




\subsection{Fallbeschreibung milde Herzfehler}

Pränatal ließen sich 30 Fälle mit milden Herzfehlern finden. Davon zeigten 96,7 \% (n=29) unauffällige kardiale Einstellungen in der frühen fetalen Herzuntersuchung (FFH). Der eine Fall mit auffälligen kardialen Einstellungen in der FFH stellte sich wie folgt dar s. Tab. 9:

Tab. 9: Fall mit mildem Herzfehler

\begin{tabular}{|c|c|c|c|c|c|c|c|c|c|}
\hline Fall & $4 \mathrm{~KB}$ & $\begin{array}{l}\text { LV } \\
\text { OT }\end{array}$ & $\begin{array}{l}\text { RV } \\
\text { OT }\end{array}$ & GA & Diagnose frühe FD & Diagnose im Verlauf & $\begin{array}{l}\text { Genetische } \\
\text { Untersuchung }\end{array}$ & $\begin{array}{l}\text { Diagnose } \\
\text { postnatal/postmortal }\end{array}$ & Outcome \\
\hline 1 & 0 & $\mathrm{P}$ & $\mathrm{P}$ & $13+5$ & $\begin{array}{l}\text { Vd.a. Aortenbogenanomalie + massiver Hydrops s. } \\
\text { Abb. } 34\end{array}$ & $\begin{array}{l}\text { Keine weitere } \\
\text { Untersuchung }\end{array}$ & $47 \mathrm{XX}+21$ & Diagnose bestätigt & $\begin{array}{l}\text { Abruptio } 17+4 \\
\text { SSW }\end{array}$ \\
\hline
\end{tabular}

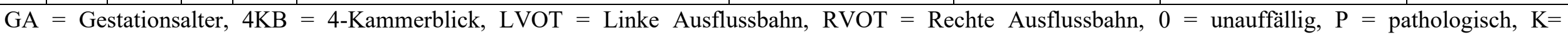
kontrollbedürftig, $\mathrm{NB}=$ nicht beurteilbar

Unter die in der Feindiagnostik detektierten Fälle leichter Herzfehler $(\mathrm{n}=$ 29) zählen: Trikuspidalklappeninsuffizienz (n = 4), Trikuspidalklappendysplasie $(\mathrm{n}=1), \operatorname{ARSA}(\mathrm{n}=11)$, kleiner muskulärer VSD $(\mathrm{n}=9)$, rechtsverlaufender Aortenbogen $(\mathrm{n}=3)$, links persistierende vena cava superior $(\mathrm{n}=1)$. In einem Fall mit leichtem Herzfehler (rechtsverlaufender Aortenbogen) wurde ein Di-George-Syndrom diagnostiziert. Diese Schwangerschaft wurde in der $24+3$ SSW beendet. 


\section{Bilddokumentation}

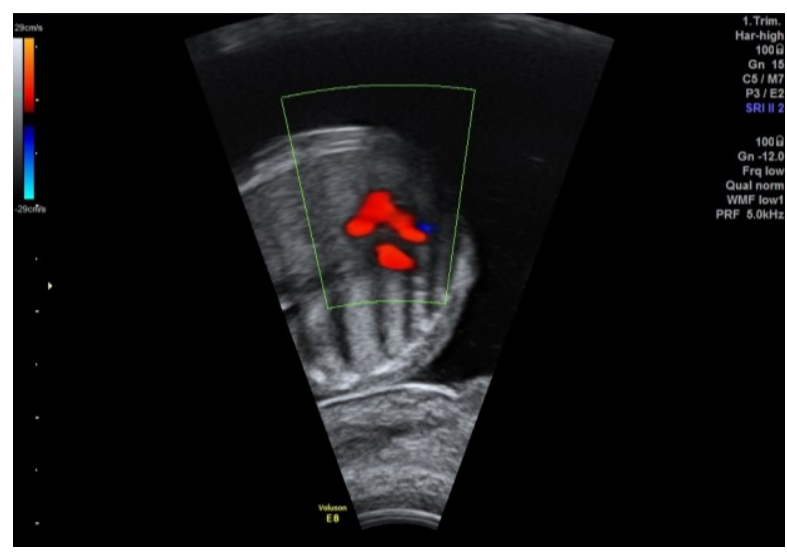

Abb. 


\subsection{Vergleich der kardialen Ultraschallbefunde}

\subsubsection{Frühe fetale Herzuntersuchung und die Echokardiographie ab der 18. SSW bei Feten} mit schweren oder moderaten Herzfehlern

Die aus den 3 Einstellungen der FFH entstandenen konkreten Diagnosen waren zu 28,5 \% in der Feindiagnostik reproduzierbar. 28,5 \% der in der FFH als kontrollbedürftig eingeschätzten Fälle konnten ebenfalls in der später erfolgten Echokardiographie konkretisiert werden. In 2 Fällen (9,5 \% der Fälle) s. Tab. 8: Fallnummer 11 und 17, in denen die Diagnosen der FFH und späteren Echokardiographie nicht übereinstimmten, war die frühe fetale FD als unauffällig eingeschätzt worden und erst in der späteren echokardiographischen Untersuchung ein schwerer oder moderater Herzfehler zu finden. In 33,3 \% der Fälle war kein Vergleich der pränatalen Diagnosen möglich, da die Schwangerschaft vor der zweiten echokardiographischen Untersuchung beendet wurde. 2 der 7 Fälle wurden jedoch paidopathologisch bestätigt s. Abb. 35 .

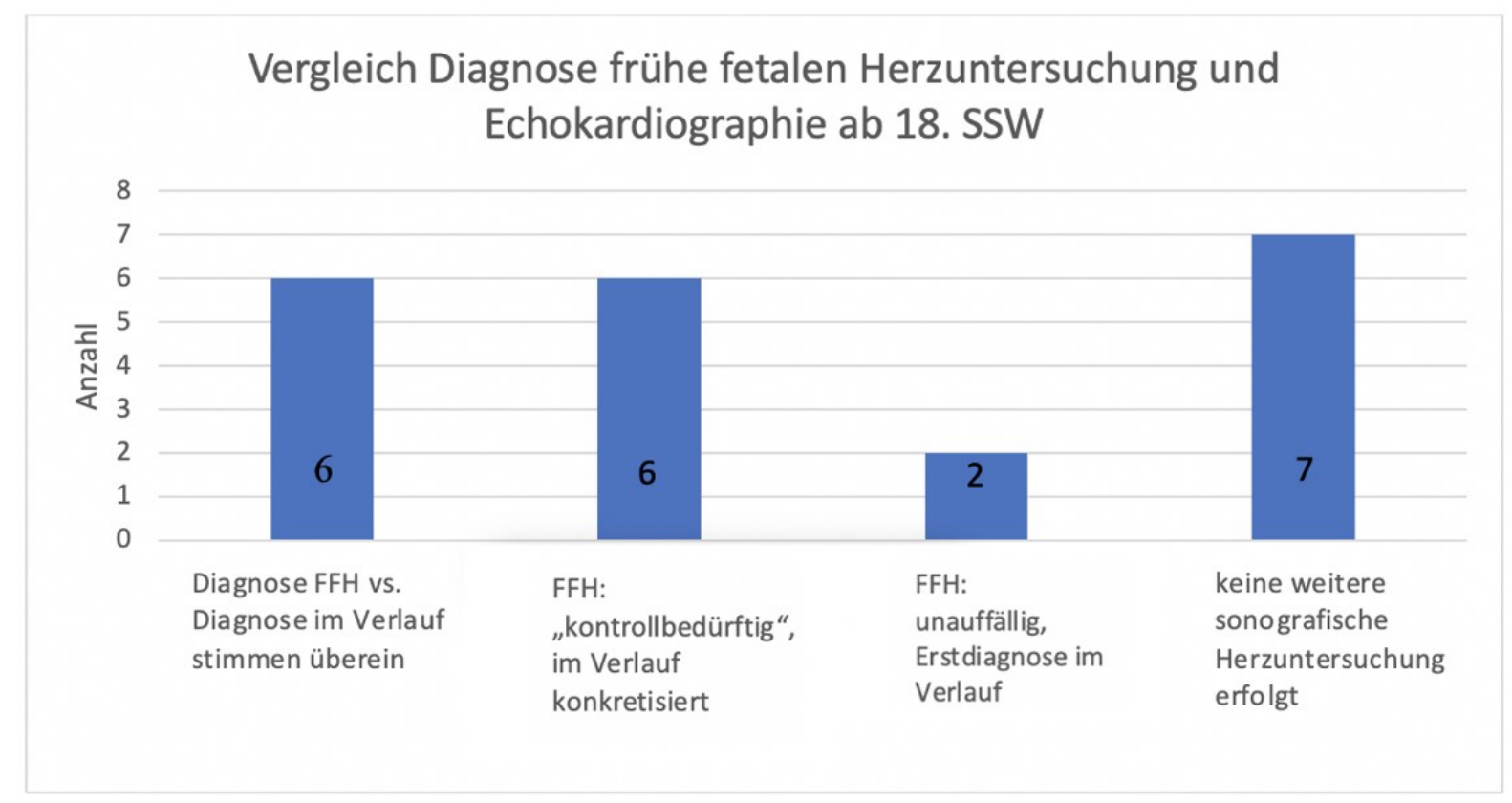

Abb. 35: Vergleich der kardialen Diagnosen in der FFH (frühen fetalen Herzuntersuchung) und Echokardiographie ab 18. SSW bei Feten mit schwerem oder moderatem Herzfehler 
6.10.2 Frühe fetale Herzuntersuchung und die Echokardiographie ab der 18. SSW im gesamten Kollektiv

Von den 1183 untersuchten Feten hatten 1076 sowohl unauffällige Einstellungen in der FFH als auch unauffällige Ergebnisse in der Echokardiographie im zweiten Trimenon.

Die 107 verbleibenden auffälligen Fälle, in denen entweder die kardialen Einstellungen im Ersttrimesterscreening als auffällig (,pathologisch/kontrollbedürftig/nicht beurteilbar“) oder die spätere Echokardiographie als auffällig eingeschätzt wurden, teilen sich wie folgt auf s. Abb. 36.

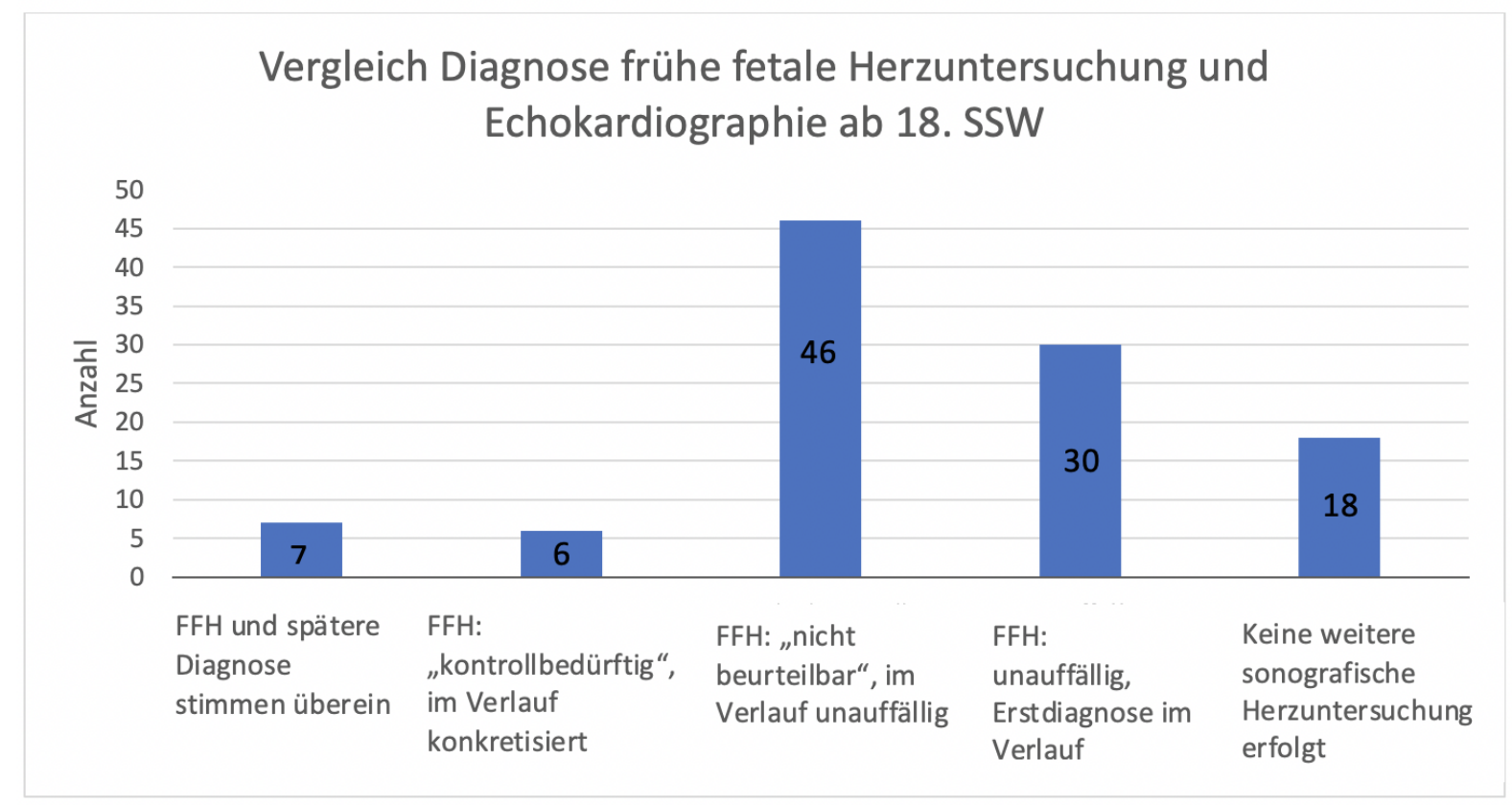

Abb. 36: Vergleich der kardialen Diagnosen in der FFH (frühen fetalen Herzuntersuchung) und in der Echokardiographie ab der 18. SSW bei allen Fällen, in denen entweder Ersttrimesterscreening als auffällig (,pathologisch/kontrollbedürftig/nicht beurteilbar") oder die spätere Echokardiographie als auffällig eingeschätzt wurde

\subsection{Postnatales Outcome}

\subsubsection{Feten mit schweren oder moderaten Herzfehlern}

Eine postnatale Echokardiographie oder paidopathologische Untersuchung lag in 15 der 21 Fälle $(71,4 \%)$ mit schwerem oder moderatem Herzfehler vor. Davon wurden 12 der 21 Diagnosen bestätigt (57,1\%). Die 3 Fälle, die postnatal nicht bestätigt wurden, waren die in Tabelle 8 aufgeführten Fälle 2, 3 und 7. 


\subsubsection{Feten mit milden Herzfehlern}

In 2 Fällen mit Trikuspidalinsuffizienz war der Ultraschallbefund (ab der 25. und 28. SSW) wieder unauffällig. In 26 der übrigen 28 Fälle konnten Daten zum weiteren Outcome der Kinder erhoben werden, jedoch lag eine Echokardiographie bzw. eine paidopathologische Untersuchung nur in 17 Fällen (60,7 \%) vor. Davon wurden 7 Diagnosen bestätigt, bei einem Fall ergab sich zusätzlich zu dem pränatal diagnostizierten rechtsverlaufenden Aortenbogen ein subaortaler VSD und in 9 Fällen konnte der pränatal diagnostizierte Herzfehler postnatal echokardiographisch nicht nachgewiesen werden s. Tab. 10.

Tab. 10: postnatal nicht bestätigte milde Herzfehler

\begin{tabular}{|l|l|}
\hline Pränatale Diagnose & Anzahl Fälle (postnatal nicht bestätigt) \\
\hline Trikuspidalklappendysplasie & 1 \\
\hline ARSA & 4 \\
\hline Kleiner muskulärer VSD & 3 \\
\hline Persistierende linke Vena cava superior & 1 \\
\hline
\end{tabular}

\subsection{Pränatal nicht diagnostizierte Herzfehler}

In dem Gesamtkollektiv von 1183 Feten wurden bei 1132 pränatal keine kardialen Auffälligkeiten gesehen. Das postnatale Outcome konnte für 598 Fälle (52,8 \%) erhoben werden. 15 Fälle zeigten eine pränatal nicht erkannte kardiale Auffälligkeit s. Tab. 11. Die Untersuchungsbedingungen waren hier bei 4 Fällen eingeschränkt (26 \%).

Tab. 11: postnatal erstdiagnostizierter Herzfehler

\begin{tabular}{|l|l|}
\hline Kardiale Auffälligkeit & n \\
\hline Kleiner VSD & 4 \\
\hline PFO & 8 \\
\hline ASD & 1 \\
\hline Kardiale Arrhythmien & 1 \\
\hline Bikuspide Aortenklappe & 1 \\
\hline
\end{tabular}




\section{Diskussion}

\subsection{Gütekriterien der kardialen Einstellungen in der frühen fetalen}

\section{Herzuntersuchung}

Die zentrale Aussage der Studie besteht in der Beantwortung der aufgestellten These „Die in der Studie beschriebenen kardialen Einstellungen helfen der Aufdeckung schwerer oder moderater fetaler Herzfehler. Kardiale Minoranomalien können erst bei späteren Untersuchungen diagnostiziert werden“.

Für die Beantwortung der These erfolgt zunächst die getrennte Betrachtung der Daten für schwere und moderate Herzfehler von denen für milde Herzfehler. Zudem ist eine Unterscheidung der kardialen Einstellungen in der FFH nach „kontrollbedürftig und pathologisch“ und „nicht beurteilbar“ sinnvoll. Die Kategorie „nicht beurteilbar“ wurde verwendet, wenn weder eine Zuordnung zu einem sicher unauffälligen noch zu einem sicher pathologischen Befund möglich war.

\section{$\underline{\text { Schwere und moderate Herzfehler }}$}

Grenzt man die kardialen Einstellungen auf „pathologisch“ oder „kontrollbedürftig“ ein, liegt die Wahrscheinlichkeit für das tatsächliche Vorliegen eines Herzfehler bei 100 \% (PPW).

Hervorragende Ergebnisse zeigen sich auch im negativen prädiktiven Wert. Falls die kardialen Einstellungen in der FFH als „unauffällig“ eingeschätzt wurden, liegt die Wahrscheinlichkeit, tatsächlich keinen schweren oder moderaten Herzfehler zu haben, bei 99,8\% (NPW).

Diese Ergebnisse tragen entscheidend zu einer differenzierten Beratung der Patientinnen und Interpretation der Untersuchungsbefunde der FFH bei.

Da es einen hohen Anteil an „nicht beurteilbaren“ Fällen gibt und sich in dieser Kategorie viele Feten ohne Herzfehler fanden, reduziert sich bei der Betrachtung von allen auffälligen (,pathologisch/kontrollbedürftig und nicht beurteilbaren“) Ergebnissen die Wahrscheinlichkeit, tatsächlich einen schweren oder moderaten Herzfehler zu haben, auf 27,9 $\%(\mathrm{PPW})$.

Der geringe positive prädiktive Wert von $<30 \%$ für ,,auffällige“ kardiale Einstellungen ist außerdem in Zusammenhang mit der geringen Prävalenz für schwere oder moderate 
Herzfehler $(1,8 \%)$ zu sehen. Bei positivem Testergebnis liegt nur zu $30 \%$ ein wirklich schwerer oder moderater Herzfehler vor.

So ist die Möglichkeit, die kardialen Einstellungen auch als „nicht beurteilbar“ einzuschätzen, eine Besonderheit der vorliegenden Studie und erhöht die Sensitivität zu Ungunsten des positiv prädiktiven Werts.

Frauen mit „nicht beurteilbaren“ fetalen kardialen Einstellungen sollten zunächst über die Wichtigkeit einer weiteren zeitnahen Kontrolluntersuchung aufgeklärt werden, ohne die Einstellungen als pathologisch einzuordnen.

Die Sensitivität für schwere und moderate Herzfehler beträgt in der vorliegenden Studie 90,4 $\%$ und die Spezifität $95,7 \%$ bei in der FFH als auffällig (,pathologisch/kontrollbedürftig/nicht beurteilbar“) beschriebenen Einstellungen. Grenzt man die kardialen Einstellungen auch hier auf „kontrollbedürftig“ oder „pathologisch“ (ohne „nicht beurteilbar“) ein, reduziert sich die Anzahl richtig erkannter Fälle, gemessen an allen Feten mit Herzfehlern, (Sensitivität) um ca. $10 \%$ auf 80,9 \%.

Somit lässt sich für den ersten Teil der o.g. These bestätigen, dass bei „pathologisch/kontrollbedürftigen“ kardialen Einstellungen in der FFH schwere oder moderate Herzfehler erkannt werden.

\section{Milde Herzfehler}

Schließt man milde Herzfehler bei der Bewertung der Sensitivität der 3 kardialen Einstellungen in der FFH mit ein, reduziert sich diese auf 39,2 \% bei einer weiterhin hohen Spezifität von 95,7\%. Dies liegt darin begründet, dass nur 1 von insgesamt 30 milden Herzfehlern in der FFH gefunden wurde. Somit kann der zweite Teil der These „Kardiale Minoranomalien können erst bei späteren Untersuchungen diagnostiziert werden“ für die vorliegende Studie ebenfalls bestätigt werden.

Bei der Bewertung der Sensitivität und des PPW muss jedoch beachtet werden, dass nicht alle schweren Herzfehler, die in einem Abort oder in einer abgebrochenen Schwangerschaft endeten, paidopathologisch bestätigt werden konnten. Dies wird genauer im Kapitel 6.4. „Vergleich von pränataler Diagnose und postnataler Untersuchung“ diskutiert. 
Auch Zidere findet in seiner Studie mit 1200 untersuchten Feten exzellente Werte für NPW (98 \%) und Spezifität (95,3 \%) für unauffällige kardiale Einstellungen von 4KB, rechtem und linkem Ausflusstrakt (Zidere et al. 2013). Die Sensitivität ist mit $84 \%$ und der PPW mit 55,8 $\%$ in einem ähnlichen Bereich wie in der hier vorliegenden Untersuchung. In einem Review von Rasiah wird eine Sensitivität von $85 \%$ und eine Spezifität von $99 \%$ für die fetalen kardialen Einstellungen angegeben (Rasiah et al. 2006).

Zusammenfassend liegt der Wert dieser Studie vor allem in den hervorragenden Ergebnissen für Sensitivität $(90,4 \%)$ und Spezifität $(95,7 \%)$ bei der Detektion von schweren oder moderaten Herzfehlern sowie dem PPW von 100 \% bei Eingrenzung der kardialen Einstellungen auf ,pathologisch oder kontrollbedürftig““.

Zudem ergibt sich aus der Spezifität, auch bei der Fragestellung nach milden Herzfehlern (95,7\%), eine hohe Wahrscheinlichkeit für das tatsächliche Vorliegen eines gesunden fetalen Herzens bei unauffälligen kardialen Einstellungen.

Bei Feten mit auffälligen (,pathologisch/kontrollbedürftig und nicht beurteilbaren“) kardialen Einstellungen sollte sich eine frühe fetale Echokardiografie anschließen.

\subsection{Vergleich der kardialen Diagnose in der frühen FD und in der}

\section{Echokardiographie ab der 18. SSW bei Feten mit Herzfehlern}

Die aufgestellte These „Ein unauffälliges Ergebnis der kardialen Diagnostik im ersten Trimenon ist bei entsprechender Indikation kein Ersatz für eine Feindiagnostik im zweiten Trimenon“ lässt sich in der vorliegenden Studie für milde Herzfehler bestätigen. So hatten unter den 30 Feten mit milden Herzfehlern $96 \%$ eine unauffällige Untersuchung im ersten Trimester.

In der Gruppe mit schweren und moderaten Herzfehlern muss für die Überprüfung der These eine Aufschlüsselung der Fälle erfolgen, da vor allem die o.g. hohe Spezifität sowie der hohe NPW dieser These entgegensteht. So sprechen diese Zahlen dafür, dass die in der frühen FD als gesund eingeschätzten Feten tatsächlich keinen Herzfehler haben. Damit könnte theoretisch auf eine weiterführende Diagnostik verzichtet werden.

In einem Drittel der 21 Fälle mit schweren oder moderaten Herzfehlern konnte die bereits in der frühen FD gestellte Diagnose zu einem späteren Zeitpunkt bestätigt werden. Von großer Bedeutung ist jedoch die mögliche Dynamik dieser Herzfehler, welche die Prognose und damit auch das geburtshilfliche Management beeinflussen kann. 
Deshalb müssen auch Schwangere mit früh diagnostizierten fetalen Herzfehlern in regelmäßiger sonografischer Kontrolle verbleiben (Smrcek et al. 2006).

So muss zum Beispiel bei einer frühen Diagnose einer TGA oder eines HLHS überprüft werden, ob sich ein restriktives Foramen ovale in utero entwickelt. Dies würde das Risiko für postnatale Komplikationen erhöhen (Talemal und Donofrio 2016).

Außerdem ist es so möglich, weitere anatomische Fehlbildungen aufzudecken, welche prognostisch relevant sein können.

Bei einem weiteren Drittel der Feten mit schweren und moderaten Herzfehlern wurden in der vorliegenden Arbeit die kardialen Einstellungen als „kontrollbedürftig“ eingeschätzt. Diese Einschätzung wurde in der Echokardiographie ab der 18. SSW konkretisiert. Die Konkretisierung ist für die Prognose und ggf. Geburtsplanung unverzichtbar und ersetzt somit in diesen Fällen ebenso keinesfalls die spätere fetale Herzuntersuchung.

Bei zwei Fällen mit schwerem CHD wurde sogar ein unauffälliges Ergebnis im Ersttrimesterscreening gesehen. Bei beiden Fällen wurde im zweiten Trimenon eine dilatative Kardiomyopathie gefunden, die im 1. Trimenon noch gar nicht diagnostiziert werden konnte. In einem Fall bestand zusätzlich eine schwere Plazentainsuffizienz.

Fetale Kardiomyopathien können sich auch durch eine Plazentainsuffizienz (Sauerstoff- und Nährstoffmangel), und somit erst im Laufe der späteren Schwangerschaft entwickeln (Trines und Hornberger 2004).

Fetale Anomalien können hinsichtlich ihrer Entdeckung im ersten Trimenon nach der Studie von Syngelaki an 44859 chromosomal unauffällige Schwangerschaften in der 11-13. SSW in 3 Gruppen unterteilt werden (Syngelaki et al. 2011): 1. Anomalien, die in der frühen FD erkannt werden müssen, 2. Anomalien, die nicht erkannt werden können, und 3. Anomalien, die partiell erkannt werden könnten.

Das Review von Khalil und Nicolaides analysiert unter anderem die Detektionsrate (DR) einzelner schwerer CHD in der frühen fetalen Herzuntersuchung. So variiert die DR von $51 \%$ für HLHS, 16 \% für CoA und 18 \% für TOF und TGA (Khalil und Nicolaides 2013).

Auch Syngelaki findet Detektionsraten von $50 \%$ für DORV, HLHS und TGA bis zu $0 \%$ für VSD sowie 0 \% für Pulmonal- und Aortenstenosen in der FFH (Syngelaki et al. 2011).

Die Ursache dafür, dass Anomalien im ersten Trimenon nicht diagnostiziert werden können, liegt zum einen im Zeitpunkt, zu dem die Anomalie phänotypisch auffällig wird. Zum anderen gibt es auch Anomalien, die sich erst später entwickeln (Ficara et al. 2020). Diese beiden Umstände können auf Herzfehler zutreffen. 
Die oben genannten Faktoren sind Gründe für die in der Literatur angegebene relativ niedrige DR für schwere CHDs. In einem Review des Jahres 2013 mit 24 Studien variiert die DR schwerer CHDs im frühen Anomalie Scan in Screeningstudien zwischen 2,5 - 53 \% (in der größten darunter eingeschlossenen Studie mit ca. 45.000 Einlingsschwangerschaften mit einer DR von 34 \%) (Khalil und Nicolaides 2013).

Die breite Spannbreite der DR macht zudem erneut deutlich, dass es fest etablierter Protokolle als Mittelweg zwischen Screening und ausführlicher Echokardiographie bedarf.

Zusammenfassend lässt sich demzufolge auch für schwere oder moderate Herzfehler bestätigen, dass das Ersttrimesterscreening trotz unauffälligem Ergebnis die spätere Echokardiographie nicht ersetzen kann. Auch wenn die hohe Spezifität und der hohe NPW belegen, dass die Wahrscheinlichkeit, bei unauffälligen kardialen Einstellungen in der frühen FD noch einen schweren oder moderaten Herzfehler zu finden, relativ gering ist.

\subsection{Kardiale Einstellung in der frühen FD - „nicht beurteilbar“}

In der vorliegenden Arbeit konnte in jedem der 1183 untersuchten Fälle eine Aussage zu 4Kammerblick, rechter und linker Ausflussbahn getroffen werden. Es erfolgte die Einteilung in „,unauffällig“ sowie auffällig: ,,pathologisch“, „,kontrollbedürftig“ oder „nicht beurteilbar“.

Die Gründe für die Einschätzung als „nicht beurteilbar“ sind eng mit der Frage nach der Untersuchungsbedingung verbunden.

In 52 von 1183 untersuchten Fällen (4,4 \%) wurde mindestens eine der drei kardialen Einstellungen als „,nicht beurteilbar“ eingeschätzt. Insgesamt zeigten die Fälle mit „nicht beurteilbaren“ Einstellungen nur in geringer Zahl einen Herzfehler (4KB zu $0 \%$ und L/RVOT zu 7,7 \%). Die darunter gefundenen 4 Fälle mit Herzfehlern wurden den schweren oder moderaten Herzfehlern zugeordnet, wobei hier in 2 von 4 Fällen der 4KB als eindeutig „pathologisch“ beschrieben wurde und nur die Ausflusstrakte „nicht beurteilbar“ waren.

Deshalb sollte eine kardiale Einstellung, die im ersten Trimenon als „nicht beurteilbar“ eingeschätzt wird, in jedem Fall zu einem späteren Zeitpunkt bzw. mittels früher Echokardiographie kontrolliert werden.

In der Einzelbetrachtung aller als auffällig (,pathologisch/kontrollbedürftig/nicht beurteilbar") eingeschätzten Fälle wurde der $4 \mathrm{~KB}$ mit $44 \%$ deutlich weniger als die Ausflusstrakte mit $80 \%$ als „nicht beurteilbar“ eingeschätzt. Die Untersuchungsbedingungen waren in beiden Gruppen in ca. $60 \%$ eingeschränkt. Dies lässt die Vermutung zu, dass der 
4KB auch bei eingeschränkten Untersuchungsbedingungen besser darzustellen ist als die Ausflusstrakte.

In einer Machbarkeitsstudie zur Basisherzuntersuchung im ersten Trimenon, in dem 4KB und Dreigefäßblick untersucht wurden, zeigt sich ebenfalls, dass der 4KB im Vgl. zum Dreigefäßblick in einer höheren Anzahl zufriedenstellend darstellbar war (86 \% vs. 79 \%) (Quarello et al. 2017).

So nutzt auch Syngelaki lediglich den 4KB zur Einschätzung des fetalen Herzens von nicht speziell für die fetale Echokardiographie ausgebildeten Untersuchern (Syngelaki et al. 2011). Hutchinson (Hutchinson et al. 2017) berichtet sogar von einer erfolgreichen Einstellung des 4KB von $98 \%$ in der 11. SSW (Hutchinson et al. 2017).

Die Untersuchungsbedingung hat in der hier vorliegenden Studie in der FFH einen signifikanten Einfluss auf die Bewertung der kardialen Parameter als „,nicht beurteilbar“ ( $\mathrm{p}=$ $<0,001)$.

Gründe für die eingeschränkten Untersuchungsbedingungen, welche in 251 Fällen vorlagen, waren Adipositas, Retroflexio uteri, Vor-Operationen, Vorderwandplazenta und eine anhaltend ungünstige fetale Position.

Bei 220 von insgesamt 251 Fällen (87\%) mit eingeschränkten Untersuchungsbedingungen war Adipositas allein oder in Kombination mit anderen Faktoren ursächlich.

Laut RKI ist die Hälfte der Frauen (53\%) in Deutschland übergewichtig (RKI 2014). Auch weltweit steigt die Anzahl adipöser Menschen. So wird es immer mehr Aufgabe von Pränataldiagnostikern, die Problematik eines erhöhten Fehlbildungsrisikos (Persson et al. 2017) mit verminderter Diagnosesicherheit zu vereinen.

Ein erhöhter BMI der Schwangeren ist mit einer niedrigeren pränatalen Diagnoserate assoziiert (Dashe et al. 2009, Aagaard-Tillery et al. 2010).

Die Untersuchungsgenauigkeit wird durch die höhere Fettschicht herabgesetzt. Dies wurde zum Beispiel in einer Studie der NBDPS (National Birth Defects Prevention Study) zu orofazialen Fehlbildungen bestätigt (Johnson et al. 2009).

Auch in der hier vorliegenden Studie liegt der durchschnittliche BMI von $24,7 \mathrm{~kg} / \mathrm{m}^{2}$ bei Müttern von Feten ohne Herzfehler und $25,6 \mathrm{~kg} / \mathrm{m}^{2}$ bei Müttern von Feten mit Herzfehlern gleichermaßen an der Grenze zu der durch die WHO definierten Präadipositas (James et al. 2000) und ist damit als relativ hoch anzusehen. 
Eine Möglichkeit zur Verbesserung der Darstellung fetaler Strukturen und damit einer Reduktion der als ,nicht beurteilbar“ eingeschätzten Fälle auch bei adipösen Schwangeren ist die kombinierte Untersuchung von Transabdominaler (TA) und Transvaginaler (TV) Untersuchung.

Vorteil der kombinierten Methode ist die genauere Auflösung der transvaginalen Untersuchung, mit jedoch geringerer Akzeptanz der Schwangeren (Abu-Rustum et al. 2010, Ebrashy et al. 2010) und geringerer Manövrierbarkeit des Schallkopfs (McAuliffe et al. 2005).

Insgesamt liegt die Rate an kombiniert oder transvaginal durchgeführten Untersuchungen in dem Gesamtkollektiv der vorliegenden Studie mit $13 \%$ relativ niedrig. Andererseits ist die Ersttrimesteruntersuchung im Mittel bei 12+3 SSW oberhalb der empfohlenen Grenze für TV oder kombinierte Untersuchungen. So wird in der Literatur für die fetale Untersuchung vor der 11. SSW ein transvaginaler Ultraschall empfohlen (Hutchinson et al. 2017). Nach der 12. SSW kann das fetale Herz suffizient mittels transabdominalem Ultraschall untersucht werden (Hernandez-Andrade et al. 2017). Transvaginaler Ultraschall wird dann zur Darstellung der fetalen kardialen Strukturen nicht mehr empfohlen, da das Herz zu weit von der Zervix entfernt ist (Smrcek 2006, Smrcek et al. 2006).

Weitere Gründe für eine trotz kombinierter Untersuchung (TV/AV) nicht ausreichende Darstellung fetaler Strukturen sind eine Vorderwandplazenta, lange Zervix sowie eine erhebliche Adipositas. So wurden auch in der hier vorliegenden Studie in $25 \%$ der Fälle mit eingeschränkter Untersuchungsbedingung trotz kombinierter Untersuchung (TV/TA) die kardialen Einstellungen als ,nicht beurteilbar“ eingeschätzt.

\subsection{Vergleich von pränataler Diagnose und postnataler Untersuchung}

Eine Zielstellung der Arbeit lag in der Untersuchung des weiteren Verlaufs der Schwangerschaft insbesondere bei Feten mit Herzfehlern.

In $71,4 \%$ der Fälle (15/21) mit schwerem oder moderatem Herzfehler lag eine postnatale Echokardiographie oder paidopathologische Untersuchung vor. In 3 Fällen (Tab. 8 Fall Nr. 2, $3,7)$ zeigte sich eine Diskrepanz zwischen der pränatal gestellter Diagnose und dem Ergebnis der postnatalen Untersuchung.

Im Fall 7 wurde pränatal ein DORV vermutet und postnatal eine Fallot-Tetralogie (TOF) diagnostiziert. Ein DORV kann als eine Variation der TOF angesehen werden, bei der das 
Ausmaß der Rechtsverlagerung der Hauptschlagader größer ist. Hier handelt es sich um Fehlbildungen, die pränatal nicht immer sicher zu unterscheiden sind, deren Differenzierung jedoch primär ohne Konsequenz für die Geburtsplanung bleibt.

Postnatal stehen weitere diagnostische Möglichkeiten (CT/MRT/Herzkatheter) zur Verfügung, um den Befund in seinem hämodynamischen Ausmaß weiter zu qualifizieren. So können die Notwendigkeit und das Ausmaß einer Therapie bestimmt werden. Der Anspruch an die pränatale Diagnose sollte Genauigkeit sein, aber entscheidend ist die aus der pränatalen Diagnose resultierende Weichenstellung für das geburtshilfliche Management, um dem Kind optimale Bedingungen für die postnatale Versorgung zu ermöglichen.

Fall 2 war auch im intradisziplinären Konsilium unter Hinzuziehung mehrerer Experten nicht eindeutig zu diagnostizieren. Hier hatten Mutter und Fet genetisch bestätigt ein Holt-OramSyndrom. Prä- und postnatal identisch ist lediglich die Beschreibung der schmalen Aorta. Gründe hierfür könnten die mit dem Syndrom einhergehenden weiteren multiplen Fehlbildungen sowie die Komplexität des Herzfehlers sein, der die Einordnung in definierte Fehlbildungen erschwert.

Im Fall 3 wurde eine rechtsventrikuläre Myokardhypertrophie vermutet, welche postnatal nicht bestätigt wurde. Auch in der FFH war diese noch nicht beschrieben. Die dort auffälligen (,nicht beurteilbaren“) Ausflussbahnen haben keine Assoziation zu einem später auffälligen rechtsventrikulären Myokard.

In 7 der 21 Fällen mit schwerem oder moderatem CHD konnte aufgrund einer beendeten Schwangerschaft keine spätere Echokardiographie durchgeführt werden.

Die Diagnosen wurden jedoch bei 2 von 7 dieser Fälle postnatal durch eine paidopathologische Untersuchung bestätigt.

Dies deckt sich mit weiteren Studien zur frühen fetalen Herzuntersuchung, in denen nicht jeder Fall postmortem bestätigt werden konnte (Krapp et al. 2011, Zidere et al. 2013).

Die fehlenden postnatalen Bestätigungen sind unter anderem auf ein zu frühes Gestationsalter zurückzuführen, in dem eine adäquate Herzuntersuchung postmortem nicht möglich war, und auch auf den Wunsch der Familie, auf eine pathologische Untersuchung zu verzichten.

Die Herzfehler dieser Feten wurden dennoch als schwerere oder moderate Herzfehler klassifiziert, da die Untersuchungen teilweise durch weitere Spezialisten der DEGUM Stufe III bestätigt wurden und auch chromosomale Störungen mit dafür typischen Herzfehlern assoziiert waren. 
Zudem kann man anhand der Bilddokumentation s. Abb. 25 - 33 vor allem in Fall 10 (s. Abb. 29), Fall 16 (s. Abb. 31) und Fall 21 (s. Abb. 33) bildmorphologisch beweisen, dass es sich um den diagnostizierten Herzfehler handelt.

Durch dieses Vorgehen könnten jedoch theoretisch falsch positive Ergebnisse, die in den vorliegenden Daten nicht gefunden wurden, übersehen werden.

Im Fall 13 wurde das Herz nach Abort in der 13. SSW nicht pathologisch untersucht. Damit wird dieser Fall in der Statistik als nicht bestätigt gewertet. Man kann allerdings vermuten, dass bei nachgewiesener Trisomie 13 auch der im Ultraschall diagnostizierte Herzfehler vorlag.

Auch in den 2 Fällen Nr. 18 und Nr. 21 (s. Tab 8) blieben die Feten nach Schwangerschaftsabbruch ohne pathologische Untersuchung. Da hier jedoch ein AVSD bzw. VSD diagnostiziert wurde und es sich somit um eine für die nachgewiesene Chromosomenstörung Trisomie 21 typischen Herzfehler handelt (Abuhamad und Chaoui 2010), ist dieser als sehr wahrscheinlich vorliegend anzusehen.

Unter den 30 pränatal diagnostizierten milden Herzfehlern waren 2 Fälle mit Trikuspidalklappeninsuffizienz nach der zweiten Echokardiographie im Verlauf wieder unauffällig. Bekannt ist, dass eine Trikuspidalklappeninsuffizienz häufig nur passager auftritt (Messing et al. 2005).

Unter den übrigen 28 Fällen lag in 17 Fällen (60,7 \%) das Ergebnis einer postnatalen Echokardiographie oder paidopathologischen Untersuchung vor.

In 7 der 17 Fälle wurden die pränatal gestellten Diagnosen bestätigt, bei einem Fall ergab sich zusätzlich zu dem pränatal diagnostizierten rechtsverlaufenden Aortenbogen ein subaortaler VSD und in 9 Fällen konnte der pränatal diagnostizierte Herzfehler postnatal echokardiographisch nicht nachgewiesen werden. Dies betraf folgende Anomalien:

Trikuspidalklappendysplasie $(n=1)$, ARSA $(n=4)$, kleiner muskulärer VSD $(n=3)$ und eine links persistierende Vena Cava superior $(n=1)$.

Gründe für das fehlende Outcome der o.g. Fälle waren ein fehlender Rücklauf der Patientinnen oder auch nicht erfolgte postpartale Diagnostik.

In der Praxis, in der die Daten für diese Arbeit akquiriert wurden, wird jeder Schwangeren, bei der eine fetale Anomalie festgestellt wird, ein Rücklaufformular ausgehändigt, mit der Bitte, dies nach der Geburt des Kindes an die Praxis zurückzusenden, was häufig unterlassen wird. 
Postnatale Daten von den Geburtskliniken zu bekommen, ist nach den geltenden Datenschutzrichtlinien ohne Einverständnis der Schwangeren nicht möglich. Diese Faktoren behindern ein vollständiges Follow-up. Es sollte zur Verbesserung der Diagnosestellung ein zuverlässiges Feed-Back System etabliert werden, das es erlaubt, die Diagnosen zu verifizieren.

Ein weiterer Grund für die fehlende Bestätigung milder Herzfehler durch eine postnatale Echokardiographie ist auch hier die mögliche Dynamik von Herzfehlern. So können sich, neben dem o.g. passageren Auftreten einer TI, zum Beispiel auch Ventrikelseptumdefekte spontan verschließen. In der Literatur werden eine spontane Verschlussrate in Utero von $5 \%$ - $48 \%$ (Gomez et al. 2014, Chau et al. 2018) und eine Verschlussrate von ca. $44 \%$ innerhalb des ersten Lebensjahres angegeben (Axt-Fliedner et al. 2006). Diese Zeitspanne liegt in unserem Follow-up-Zeitraum und kann somit bei der telefonischen und schriftlichen Befragung der Mütter zu dem aktuellen Herzfehler ihres Kindes zu falschen Aussagen führen.

15 Feten zeigten pränatal nicht erkannte kardiale Auffälligkeiten, welche alle den milden Herzfehlern zugeordnet werden können. Diese sind allerdings in der Statistik nicht berücksichtigt, da die vorliegende Arbeit primär auf den Vergleich der frühen fetalen Herzuntersuchung mit der Echokardiographie ab der 18. SSW zielt und den Schwangerschaftsverlauf beschreiben soll.

Insgesamt muss die o.g. Zahl von 15 Feten sogar mit Vorsicht interpretiert werden und liegt eventuell noch höher, da nur bei 52,8 \% der untersuchten Feten ohne pränatal diagnostizierte Herzfehler ein postnatales Outcome vorlag.

Es kann allerdings sicher gesagt werden, dass in den o.g. 52,8 \% kein schwerer oder moderater Herzfehler übersehen wurde.

\subsection{Untersuchungskollektiv, Risikofaktoren und Prävalenz von kongenitalen Herzfehlern}

In dem untersuchten Kollektiv von 1183 Feten wurde bei 51 Feten pränatal ein Herzfehler diagnostiziert. Die Prävalenz von 4,3 \% liegt damit deutlich höher als die in der Literatur angegebenen Gesamtprävalenz für angeborene Herzfehler von ca. 1 \% (Ren et al. 2008, Fahed et al. 2013).

Dabei unterliegt die Prävalenz von Herzfehlern einer hohen Schwankungsbreite (Hoffman und Kaplan 2002, Bernier et al. 2010). Schließt man milde Herzfehler in die Betrachtung der 
Prävalenz ein, findet man ähnlich hohe Fallzahlen (50/1000) (Benson 2002) wie in der hier vorliegenden Studie. Dennoch bleibt die Prävalenz in dieser Untersuchung erhöht, da auch die Eingrenzung der Herzfehler auf schwere oder moderate Fälle mit einer Prävalenz von 1,8 \% im Verhältnis zur Gesamtprävalenz fast doppelt so hoch liegt.

Die Gründe für die deutlich höhere Prävalenz in den vorliegenden Daten sind vielschichtig und werden im Folgenden diskutiert.

Zunächst bezieht sich die Krankheitshäufigkeit der in der Literatur angegebenen Herzfehler auf lebendgeborene Kinder. Da in der vorliegenden Studie auch der Verlauf der pränatal diagnostizierten Herzfehler untersucht wird, bleiben auch die nicht lebend geborenen Kinder bzw. vorzeitig beendeten Schwangerschaften in die Betrachtung eingeschlossen.

Die Prävalenz der angeborenen Herzfehler bei lebendgeborenen Kindern hat sich zwar in den letzten 15 Jahren auf ca. 10/1000 stabilisiert, dennoch findet man in den letzten 50 Jahre eine deutliche Zunahme der Prävalenz der Herzfehler unter lebend geborenen Kindern (van der Linde et al. 2011). Es wird vermutet, dass die steigende Detektionsrate von vor allem leichten fetalen Fehlbildungen (Dolk et al. 2011) unter anderem auf einer Verbesserung der verwendeten Ultraschalltechnik beruht (Marek et al. 2011, Pinto et al. 2012). Als weiterer Grund für die steigende Detektionsrate wird die Spezialisierung des Untersuchers aufgeführt (Marek et al. 2011, Pinto et al. 2012). Beide Faktoren - die Untersuchung auf DEGUM Level III Niveau und der Einsatz modernster Ultraschalltechnik - können dazu führen, dass Herzfehler in der hier vorliegenden Arbeit häufiger als in der Gesamtpopulation gefunden wurden.

Die Verwendung des gleichen Ultraschallgeräts und vor allem die Untersuchung durch nur eine Untersucherin ist jedoch ein Vorteil der Studie und trägt zur Objektivierung der Ergebnisse bei.

Zudem ist die Auswahl des Patientenkollektivs kritisch $\mathrm{zu}$ betrachten, denn nicht alle Schwangeren haben die Indikation für eine frühe Feindiagnostik zwischen $11+0$ und 13+6 SSW oder für eine Feindiagnostik zwischen 18+0 und 21+6 SSW. Die Kosten für das Ersttrimesterscreening müssen die Mütter laut Bundesausschuss der Ärzte und Krankenkassen bei einer unauffälligen Schwangerschaft und Schwangerschaftsanamnese selbst tragen, so kann man annehmen, dass der Großteil der in dieser Studie eingeschlossenen Patientinnen eine Untersuchungsindikation vorwies und damit vermutlich eine Verschiebung zugunsten von Risikoschwangerschaften vorliegt. 
In einem Review mit ca. 80.000 eingeschlossenen Feten wird gezeigt, dass die Detektionsrate von Herzfehlern in Hochrisikogruppen höher ist als bei unselektierten Schwangeren (Rossi und Prefumo 2013).

Die Ursache angeborener Herzfehler bleibt in den meisten Fällen ungeklärt (Allan 1995). Es konnten jedoch Risikofaktoren als Kombination von Umweltfaktoren und genetischen Veränderungen, welche die Entstehung von Herzfehlern begünstigen, identifiziert werden (Ahmadi et al. 2020).

In dem vorliegenden Kollektiv konnte in Bezug auf keinen der bekannten Risikofaktoren, wie mütterliche Stoffwechselstörungen (Abuhamad und Chaoui 2010), mütterlicher Diabetes mellitus oder maternale Adipositas (Cedergren und Kallen 2003, Brite et al. 2014, Persson et al. 2017), ein signifikanter Unterschied zwischen den Gruppen mit und ohne Herzfehler gefunden werden.

Auch das maternale Alter, welches auch bei Feten ohne chromosomale Störungen ein Risikofaktor für angeborene Herzfehler ist (Hollier et al. 2000, Forrester und Merz 2004, Miller et al. 2011), zeigt keinen signifikanten Unterschied zwischen den Gruppen mit und ohne Herzfehler.

Der Anteil an Schwangeren $>35$ Jahre, beträgt im Kollektiv 34,7 \% und ist damit im Vergleich mit anderen Studien zur frühen fetalen Echokardiographie im NiedrigrisikoBereich einzuordnen (Becker und Wegner 2006).

Die Auswertung weiterer Risikofaktoren wie einer positiven Familienanamnese bezüglich vorliegender Herzfehler in der Familie, monochorialer Geminigravidität, der Einnahme von Medikamenten insbesondere von Antidepressiva und Antiepileptika sowie der Anwendung assistierter Reproduktionstechnologie zeigte in dieser Studie ebenfalls keinen signifikanten Unterschied zwischen den Gruppen mit und ohne Herzfehler. Ein Grund hierfür könnte die geringe Fallzahl sein.

Gesondert betrachtet werden muss bei der Einschätzung des Patientenkollektivs der Anteil an Feten mit erhöhter Nackentransparenz $(\mathrm{NT}>95$. Perzentile), da eine erhöhte Nackentransparenz einer der wichtigsten Ultraschallbefunde ist, der auf einen Herzfehler hinweist und einen Überweisungsgrund zu einer weiterführenden Untersuchung darstellt (Makrydimas et al. 2003, Iliescu et al. 2013).

So steht der o.g. hohe Anteil schwerer oder moderater kardialer Herzfehler in der vorliegenden Studie auch im Zusammenhang mit einer NT $>95$. Perzentile von insgesamt 6,8 \%. Dies liegt höher als die in anderen großen Studien zu findende NT-Prävalenz von 2,5 - 4,4 $\%$ in den Studienpopulationen (Snijders et al. 1998, Souka et al. 2005, Holzer et al. 2019). 
So lässt sich zusammenfassend sagen, dass durch die höhere Rate an Feten mit erhöhter NT im hier untersuchten Kollektiv ein höheres Risiko für Herzfehler besteht.

Bei allen anderen o.g. Risikofaktoren besteht keine signifikante Assoziation zu Herzfehlern. Dennoch sollte bei diesen Schwangerschaften nach aktueller Studienlage eine Untersuchung auf fetale Herzfehler erfolgen (Abuhamad und Chaoui 2010).

\subsection{Weitere anatomische Auffälligkeiten und chromosomale Störungen}

Von den in dieser Studie insgesamt gefundenen 51 Fällen mit Herzfehlern traten 58,8 \% isoliert auf. Unter allen Fällen mit isolierten Herzfehlern fanden sich jedoch 83,3 \% milde Herzfehler und wurden in 29 von 30 Fällen erst im zweiten Trimenon erkannt.

Unter den insgesamt 51 Fällen mit Herzfehlern wurden 20 Fälle im ersten Trimenon als auffällig beschrieben, wovon wiederum nur 5 Fälle (25\%) einen isolierten Herzfehler hatten. Somit kann der erste Teil der in dieser Arbeit aufgestellten These, dass schwere kardiale Anomalien, die im ersten Trimenon diagnostiziert werden, häufig Teil komplexer morphologischer Anomalien bzw. genetischer Fehlbildungen sind, für diese Studie bestätigt werden.

Herzfehler gehen mit einer erhöhten Rate an chromosomalen Störungen einher. Die Studie von Chaoui (Chaoui et al. 1999) mit 2716 untersuchten Feten und 203 diagnostizierten Herzfehlern berichtet bei $22 \%$ eine Chromosomenanomalie. In der hier vorliegenden Arbeit liegt die Rate mit 46 \% sogar deutlich höher, liegt aber innerhalb der in weiteren Studien angegebenen Schwankungsbreite von 29,2 \% (Jortveit et al. 2016) bis 65,8 \% (Smrcek et al. 2006) für mit Fehlbildungen assoziierte chromosomale Anomalien bei Feten mit schweren Herzfehlern.

Das Risiko für schwere Herzfehler steigt exponentiell mit der Dicke der Nackentransparenz (Atzei et al. 2005, Bhat et al. 2013). Der Anteil an Feten mit schwerem oder moderatem CHD und erhöhter Nackentransparenz ohne chromosomale Störung liegt in der vorliegenden Arbeit bei $33 \%$ und damit zwischen den in der Literatur zu findenden Angaben von 28,9\% (Martinez et al. 2010), 31 \% (Makrydimas et al. 2003) und $44 \%$ (Brooks et al. 2014).

In der vorliegenden Arbeit konnte im Vergleich aller Feten mit moderatem oder schwerem Herzfehler mit Feten ohne Herzfehler ein signifikanter Unterschied im Mittelwert der Nackentransparenz nachgewiesen werden $(3,3 \mathrm{~mm}$ versus $1,8 \mathrm{~mm} \mathrm{p}=0,001)$. 
Dies lässt sich auch in einer Studie von Mavrides (Mavrides et al. 2001) belegen. Unter 7339 untersuchten Feten fanden sich 26 Feten mit major CHD. Bei erhöhter Nackentransparenz ( $>$ 3,5 mm) wurde ein signifikant erhöhtes Risiko eines CHDs nachgewiesen.

Auch die Ergebnisse von Yang (Yang und Zhang 2014), in der 4673 Feten in der 11-14. SSW untersucht wurden, belegen, dass eine erhöhte Nackentransparenz in Kombination mit der frühen fetalen Herzuntersuchung einen Großteil der schweren Herzfehler detektieren kann.

Damit wird der auch hier vorliegende Zusammenhang zwischen Nackentransparenz und fetalen Herzfehlern bestätigt.

Sowohl die zitierten Studien als auch die Daten dieser Arbeit identifizieren bei erhöhter NT eine Subgruppe von Hochrisikopatienten für schwere Herzfehler, die einer Untersuchung des Herzens im ersten Trimenon unterzogen werden sollten.

Dies gilt jedoch nicht für Feten mit milden Herzfehlern. Im Vergleich der Nackentransparenz von Feten mit milden Herzfehlern und Feten ohne Herzfehlern konnte in der vorliegenden Arbeit kein signifikanter Unterschied gefunden werden.

Auch in der Literatur zeigt sich die erhöhte Nackentransparenz nur bei Feten mit schweren oder moderaten Herzfehlern (Makrydimas et al. 2003). Die Nackentransparenz kann nicht zur Identifizierung der Risikogruppe für leichte Herzfehler verwendet werden.

Wie die im Kapitel 6.1. diskutierten Daten belegen, erlauben die Darstellung des 4KB, der linken und rechten Ausflussbahn eine zeitnahe Einschätzung des fetalen Herzens und die frühzeitige Erfassung schwerer CHD.

Damit werden die Wartezeit und auch die psychische Belastung vor einer weiterführenden Diagnostik für Eltern und Angehörige von einem Feten mit einem erhöhten Risikoprofil reduziert.

Als weitere Marker für den CHD werden neben der NT ein abnormer Blutfluss des Ductus venosus (DV) und die Trikuspidalregurgitation (TR) genannt (Minnella et al. 2020). Dies wurde zwar in der vorliegenden Studie nicht untersucht, soll aber im Folgenden kurz erläutert werden, da diese Parameter im wissenschaftlichen Diskurs zur Früherkennung angeborener Herzfehler einen wichtigen Stellenwert einnehmen. So werden vor allem Feten mit auffälligen Ergebnissen in allen drei Parametern (NT, DV-Fluss und TR) zu einem Risikokollektiv für fetale Herzfehler gezählt. Die frühe FD sollte hier mit den in dieser Studie untersuchten 3 kardialen Einstellungen in jedem Fall durchgeführt werden. 
In der Studie von Martinez erhöht die Untersuchung des Ductus-venosus-Flusses die Detektionsrate von Herzfehlern um 11 \% gegenüber der Verwendung der Nackentransparenz allein (Martinez et al. 2010). Das relative Risiko für einen Herzfehler ist bei abnormen DVFluss höher als das Risiko bei maternalen Risikofaktoren wie Diabetes mellitus oder positiver Familienanamnese für Herzfehler. Dies führt zu der Fragestellung, ob eine detaillierte fetale Echokardiographie bei abnormem DV-Fluss indiziert ist. In einer Studie von Maiz mit 11.000 untersuchten Schwangerschaften wurde kein Vorteil von Messung des DV-Flusses bei unauffälliger Nackentransparenz gefunden (Maiz et al. 2008). Die Autoren betonen die Notwendigkeit der Kombination verschiedener Marker zur Detektion von fetalen Herzfehlern. Die ergänzende Messung der TR zu DV-Fluss und NT erhöht die Detektionsrate von schweren Herzfehlern (Minnella et al. 2020). Auch in einer Übersichtsarbeit zu den drei kombinierten Parametern Nackentransparenz, DV-Fluss und TR wird nach Zusammenschau der vorliegenden Literatur empfohlen, auch bei Feten mit TR und NT $>95$. Perzentile eine detaillierte frühe Echokardiographie durchzuführen (Geipel und Gembruch 2012).

Die Messung der TR alleine reicht jedoch nicht aus, um die Indikation für eine frühe fetale Echokardiographie zu stellen, da die Genese der TR nicht immer in einer strukturellen Anomalie zu finden ist und der Befund der TR häufig transient auftritt (Huggon et al. 2003, Karadzov Orlic et al. 2019).

\subsection{Abbruch der Schwangerschaft}

In der vorliegenden Arbeit liegt die Abbruchrate in der Gruppe mit schweren oder moderaten Herzfehlern bei 42,9 \% (9 von 21 Fällen). 6 der 9 Feten (66\%) wiesen dabei weitere teils schwere assoziierte anatomische Fehlbildungen auf.

Unter den Feten mit schwerem oder moderatem Herzfehler kam es in der hier vorliegenden Analyse bei 3 Fällen zu einem Abort. Hier konnte nicht eruiert werden, ob der Wunsch, die Schwangerschaft fortzusetzen, bestanden hätte. Alle 3 Feten hatten schwere komplexe fetale Anomalien.

Auch in der Gruppe mit milden Herzfehlern wurden zwei Schwangerschaften durch einen Abbruch beendet. Hier lagen in beiden Fällen syndromale Erkrankungen vor (Trisomie 21 und Di-George Syndrom).

Je früher die Eltern eines Feten mit schwerem Herzfehler und möglicher lebenslimitierender Prognose die Diagnose und entsprechende Beratung erhalten, desto früher können sie das Für und Wider eines Schwangerschaftsabbruchs abwägen. 
Studien zeigen, dass ein früherer Schwangerschaftsabbruch medizinisch sicherer und vor allem für die Schwangere psychisch weniger belastend ist als ein Abbruch zu einem späteren Zeitpunkt (Lawson et al. 1994, Davies et al. 2005, Daugirdaite et al. 2015).

Dies macht die Dringlichkeit der genauen Einordnung auffälliger sonografischer Befunde im ersten Trimenon deutlich.

Für die aufgestellte These: „Die frühe Diagnostik von fetalen Anomalien bringt aber auch die Gefahr einer vorschnellen Entscheidung zum Schwangerschaftsabbruch" kann in der vorliegenden Arbeit aufgrund der geringen Fallzahl keine verlässliche Antwort gegeben werden. Es ist die Tendenz $\mathrm{zu}$ erkennen, dass die Entscheidung zum Schwangerschaftsabbruch nicht vorschnell getroffen wird.

Für die Diskussion der These wird zum einen die Abbruchrate im Kollektiv frühe vs. späte Diagnose eines schweren oder moderaten Herzfehlers betrachtet. Zum anderen wird der Zeitraum zwischen Diagnosestellung und Zeitpunkt des Schwangerschaftsabbruchs diskutiert.

Unter den früh diagnostizierten schweren oder moderaten Herzfehlern liegt die Abbruchrate bei 42,9\%. Dies steht den 2 Fällen gegenüber, in denen der schwere oder moderate Herzfehler erst zu einem späteren Zeitpunkt diagnostiziert wurde. Hier endeten die Schwangerschaften in einem Abort und in einem Abbruch.

In einer Studie von Bhat wurden 35 Schwangerschaften mit Fallot-Tetralogie unter anderem nach dem Zeitpunkt des Schwangerschaftsabbruchs untersucht (Bhat et al. 2013). Das Ergebnis zeigte, dass signifikant mehr Schwangerschaften nach früher Diagnose (12 - 17 SSW) gegenüber der Gruppe mit später Diagnose (18 - 25 SSW) abgebrochen (80 vs. 33 \%) wurden. Auch in der Studie von Jicinska, in der der Verlauf von 127 Feten mit früher Diagnose eines Herzfehlers und 344 Feten mit einer späteren Diagnose eines Herzfehlers begleitet wurde, wird bestätigt, dass die frühe Detektion von Herzfehlern mit signifikanten Komorbiditäten zu einer erhöhten Schwangerschaftsabbruchrate im ersten Trimenon führt (Jicinska et al. 2017).

Diese Studien betonen aber außerdem, dass bei früher Diagnostik eine besonders intensive Beratung und Führung der Schwangeren und ihres Partners notwendig und möglich sind.

So liegt der Vorteil einer frühen Diagnosestellung darin, dass mehr Zeit bis zur fetalen Lebensfähigkeit existiert und damit die weitere Betreuung mit der nötigen Ruhe organisiert werden kann. 
In der hier vorliegenden Studie lag die durchschnittliche Zeit des Schwangerschaftsabbruchs bei Feten mit schwerem oder moderatem CHD (ausgenommen der zwei erst später auffälligen Fälle) in der 16+5 SSW und damit im Durchschnitt 31 Tage nach Diagnosestellung.

Diese Zeitspanne ist angemessen, um einer ausreichenden Bedenkzeit, Diagnosesicherung durch eine Kontrolluntersuchung, einer genetischen Untersuchung und interdisziplinärer Beratung ausreichend Raum zu geben.

Einerseits stellt die frühe Diagnosestellung Eltern von Feten mit auffälligen Herzbefunden im ersten Trimenon schon früh vor eine sehr schwierige Entscheidung, insbesondere wenn die kardialen Auffälligkeiten isoliert auftreten und eine Lebensfähigkeit bei jedoch wahrscheinlich eingeschränkter Lebensqualität zu erwarten ist.

Andererseits haben Eltern, die sich nach einer frühen Diagnosestellung für die Fortsetzung der Schwangerschaft entscheiden, mehr Zeit, um medizinische und ökonomische Vorbereitungen für die Versorgung des Kindes zu treffen.

Auch die Organisation der Geburt und der interdisziplinären Beratung und somit der bestmöglichen Versorgung des Kindes kann bei früher Diagnosestellung gut geplant werden. In der Studie von Jortveit, in der knapp 1 Millionen Feten untersucht wurden, wurde eine Reduzierung der 1-Jahresmortalität von Feten mit schweren Herzfehlern im Zeitraum von 1994-2009 dokumentiert (Jortveit et al. 2016). Hierfür genannte Gründe sind vor allem die reduzierte operative Mortalität, welche unter anderem durch eine genaue und gute Planung der postnatalen Versorgung bei pränatal bekannter Diagnose bedingt wird.

\subsection{Einordnung der Methode und Sicherheitsaspekte}

Die in der vorliegenden Studie verwendeten drei kardialen Einstellungen 4KB, LVOT und RVOT stellen eine Möglichkeit dar, um im Rahmen einer frühen Feindiagnostik einen Kompromiss zwischen einer ausführlichen Echokardiographie und einer nur auf die Darstellung des Vierkammerblicks begrenzten Untersuchung $\mathrm{zu}$ schaffen. Zur besseren Darstellbarkeit bedarf es der Anwendung von Color Doppler (CD), welche in jedem untersuchten Fall dieser Studie verwendet wurde.

Auch in der Studie von Wiechec, in der 1084 Patienten bezüglich der Aussagekraft der 2 kardialen Einstellungen $4 \mathrm{~KB}$ und 3-Gefäße-Trachea-Blick mit und ohne farbkodierter Doppler-Sonografie untersucht wurden, zeigt sich, dass die Verwendung von CD eine signifikant bessere Aufdeckungsrate von Herzfehlern hatte (Wiechec et al. 2015). 
CD ist im Vergleich zum B-Bild mit höheren Intensitäten des Ultraschalls verbunden. So wird in einer Metaanalyse der WHO (Torloni et al. 2009) sowie einem Cochrane-Review (Whitworth et al. 2010) resümiert, dass Ultraschall eine sichere Methode zur Untersuchung des Feten darstellt. Dennoch sollte die Anwendung von CD zur Vermeidung von thermaler Schädigung so gering wie möglich gehalten werden (Salvesen et al. 2011). Die ISUOG betont ebenfalls in ihrem safety statement, dass der fehlende Nachweis von Schädigung durch den Einsatz von CD nicht mit der Annahme der Ungefährlichkeit für den Feten gleichzusetzen ist. Vor allem die Anwendung von CD in einer frühen Phase der Schwangerschaft sollte nur mit entsprechender Indikation und beschränkt auf ein nötiges Maß erfolgen. Dennoch findet man in vielen Studien zur fetalen Echokardiographie in einem frühen Gestationsalter die Anwendung des CD, welche die Detektionsrate von Herzfehlern erhöht und damit als nötiger Nutzen/Risiko-Kompromiss angesehen wird (Smrcek 2006, Iliescu et al. 2013, Hutchinson et al. 2017).

Auch unter diesem Aspekt ist es wichtig, ein Protokoll mit aussagekräftigen Einstellungen zur Einschätzung der jeweiligen Organstrukturen zu etablieren. Dadurch kann die Untersuchung so kurz wie möglich und so lang wie nötig gehalten werden - ALARA-Prinzip: As Low As Reasonably Achievable (Berkhout 2015).

Es sollte zudem diskutiert werden, ob weitere kardiale Einstellungen ergänzt werden müssen, wenn die 3 oben genannten kardialen Einstellungen nicht zu einer eindeutigen Einschätzung führen.

Nachteil weiterer Ebenen ist jedoch die reduzierte Rate an erfolgreicher Visualisierung. So berichtet das Review von Hernandez-Andrade (Hernandez-Andrade et al. 2017) eine Darstellungsrate von 70-80 \% bei Aortenbogen und Blutflussmuster im Ductus venosus, 60$70 \%$ bei Trachealblick und Dreigefäßblick sowie 25 - $55 \%$ bei Pulmonalvenendarstellung im ersten Trimenon.

In der Studie von Wiechec (Wiechec et al. 2015) und in der Studie von Heling und Chaoui (KS Heling und Chaoui 2007) wurde eine sehr gute Erkennbarkeit von fetalen Herzfehlern im ersten Trimenon unter Verwendung von Dreigefäßblick und $4 \mathrm{~KB}$ in Kombination mit Farbdoppler gefunden. Die Studie von Krapp (Krapp et al. 2011) verzichtet auf eine isolierte RVOT Einstellung zugunsten des Dreigefäßblicks, da der Wechsel von 4KB zu LVOT im Dreigefäßblick endet und keine zusätzlichen Informationen durch eine extra Einstellung des RVOT zu erwarten wären. 
Die durch das frühe Gestationsalter bedingte anatomische Nähe der Herzgefäße bewirkt, dass bei der Einstellung des RVOT auch oft gleichzeitig der sogenannte „Dreigefäßblick“ zur Darstellung kommt. Dieser ist im ersten Trimenon ein „Zweigefäßblick“ und bildet den Zusammenfluss von DAB und AO mit dem typischen „V“ ab, s. zum Beispiel Kapitel 5.11, Tab. 8: Fall 9 (Abb. 28) und Fall 10 (Abb. 29) und Tab. 9 Fall 1 (Abb. 34).

In der hier vorliegenden Arbeit wurden beide Ausflusstrakte LVOT und RVOT bei Auffälligkeit („nicht beurteilbar/pathologisch/kontrollbedürftig“) immer gleich klassifiziert.

Somit kann man vermuten, dass kein Mehrwert in der Einstellung beider Ausflusstrakte besteht. Die Umstellung der kardialen Einstellung auf 4KB, LVOT und Dreigefäßblick sollte Gegenstand weiterer Untersuchungen sein.

Auch Syngelaki fordert ein Protokoll für das Ersttrimesterscreening und betont, dass es sinnvoll ist, darin einfach darzustellende anatomische Landmarken zu etablieren, an denen strukturelle Störungen festgemacht werden können (Syngelaki et al. 2011).

Zusammenfassend ist die Untersuchung des fetalen Herzens mittels den in dieser Studie verwendeten Einstellungen von $4 \mathrm{~KB}$, LVOT und RVOT unter Verwendung von CD eine Möglichkeit, um eine orientierende Einschätzung des fetalen Herzens unter Verzicht auf eine zeitaufwendige, schwierig einzustellende frühe fetale Echokardiographie vorzunehmen. 


\section{Schlussfolgerung}

Die 3 untersuchten Einstellungen 4KB, LVOT und RVOT weisen eine hohe Spezifität und Sensitivität für die Erfassung schwerer und moderater Herzfehler bereits im 1. Trimenon auf. Deshalb kann dieses Protokoll für die Untersuchung des fetalen Herzens im ersten Trimenon eingesetzt werden.

Bei als „auffällig“ eingeschätzten kardialen Einstellungen sollte bei den hier nachgewiesenen relativ niedrigen Werten für PPW eine frühe fetale Echokardiographie angeschlossen werden. Die hohen NPW geben den Eltern wiederum eine Sicherheit, dass bei unauffälligen Einstellungen in der FFH tatsächlich auch kein Herzfehler vorliegt.

Durch die Einbeziehung der Ausflussbahnen und des Color Dopplers gibt dieses Protokoll mehr Informationen als die alleinige Darstellung des 4KB im B-Bild und ist zeitlich, personell und apparativ nicht so aufwendig wie eine fetale Echokardiographie im ersten Trimenon.

Auch Risikogruppen, insbesondere Feten mit erhöhter NT, können mit diesem Protokoll im ersten Trimenon orientierend untersucht und die Feten erkannt werden, bei denen eine Indikation zur fetalen Echokardiographie besteht.

Somit ist eine orientierende Untersuchung des fetalen Herzens zu einem frühen Zeitpunkt möglich und es kann eine Weichenstellung für den weiteren Verlauf der Schwangerschaft erfolgen. Der Vorteil der frühen Diagnosestellung sind zum einen eine frühe Beratung und bestmögliche Vorbereitung für die postnatale Versorgung und zum anderen auch mehr Zeit bis zur Lebensfähigkeit des Feten.

Dennoch kann sowohl bei auffälliger als auch bei unauffälliger kardialer Untersuchung im ersten Trimenon auf eine Untersuchung zu einem späteren Zeitpunkt nicht verzichtet werden. Dies liegt vor allem an der möglichen Entwicklung eines Herzfehlers im Laufe der Schwangerschaft, der in der fetalen Herzuntersuchung im ersten Trimenon noch nicht erkennbar ist.

Auch sollte bei eingeschränkten Untersuchungsbedingungen, die häufig mit einer mangelnden Einschätzung insbesondere der Ausflusstrakte einhergehen, die Begrenzung der Anwendung beachtet werden und eine erneute Herzuntersuchung im Verlauf erfolgen. 
Limitierungen zeigt die Studie bei der Frage nach milden Herzfehlern. Diese können durch eine frühe fetale Herzuntersuchung mittels der drei untersuchten Einstellungen unseren Ergebnissen zufolge nicht entdeckt werden.

Weiteren Forschungsbedarf ergibt sich zudem aus der Frage, ob statt der Einstellung von RVOT der Dreigefäßblick in das Protokoll für die frühe fetale Herzuntersuchung einbezogen werden sollte. Außerdem sollte die Anwendung der drei Einstellungen in Zentren mit unterschiedlicher Untersucherqualifikation und mit einem homogenen Risikokollektiv durchgeführt werden, um die Ergebnisse der vorliegenden Studie in einer nationalen Empfehlung festzuhalten. 


\section{Literatur- und Quellenverzeichnis}

Aagaard-Tillery KM, Flint Porter T, Malone FD, Nyberg DA, Collins J, Comstock CH, Hankins G, Eddleman K, Dugoff L, Wolfe HM, D'alton ME. 2010. Influence of maternal BMI on genetic sonography in the FaSTER trial. Prenat Diagn 30(1): 14-22.

Abdul-Khaliq HB, Felix. 2011. Angeborene Herzfehler: Die Diagnose wird häufig zu spät gestellt. Deutsches Ärzteblatt 108(31-32): A-1683 / B-1433 / C-1429.

Abu-Rustum RS, Daou L, Abu-Rustum SE. 2010. Role of first-trimester sonography in the diagnosis of aneuploidy and structural fetal anomalies. J Ultrasound Med 29(10): 1445-1452.

Abuhamad A, Chaoui R. 2010. A parctical guide to fetal echocardiography. Philadelphia: Lippincott Williams and Wilkins, a Wolters Kluwer business, 4-5

Ahmadi A, Gharipour M, Navabi ZS, Heydari H. 2020. Risk factors of congenital heart diseases: A hospital-based case-control study in Isfahan, Iran. ARYA Atherosclerosis 16(1): $1-6$.

Allan LD. 1995. Echocardiographic detection of congenital heart disease in the fetus: present and future. Br Heart J 74(2): 103-106.

Alverson CJ, Strickland MJ, Gilboa SM, Correa A. 2011. Maternal smoking and congenital heart defects in the Baltimore-Washington Infant Study. Pediatrics 127(3): e647-653.

Apitz J. 1998. Pädiatrische Kardiologie Erkrankungen des Herzens bei Neugeborenen, Säuglingen, Kindern und Heranwachsenden. Heidelberg: Steinkopff-Verlag, 17-21. Atz AM, Travison TG, Williams IA, Pearson GD, Laussen PC, Mahle WT, Cook AL, Kirsh JA, Sklansky M, Khaikin S, Goldberg C, Frommelt M, Krawczeski C, Puchalski MD, Jacobs JP, Baffa JM, Rychik J, Ohye RG, Pediatric Heart Network I. 2010. Prenatal diagnosis and risk factors for preoperative death in neonates with single right ventricle and systemic outflow obstruction: screening data from the Pediatric Heart Network Single Ventricle Reconstruction Trial. J Thorac Cardiovasc Surg 140(6): 1245-1250.

Atzei A, Gajewska K, Huggon IC, Allan L, Nicolaides KH. 2005. Relationship between nuchal translucency thickness and prevalence of major cardiac defects in fetuses with normal karyotype. Ultrasound Obstet Gynecol 26(2): 154-157. 
Axt-Fliedner R, Schwarze A, Smrcek J, Germer U, Krapp M, Gembruch U. 2006. Isolated ventricular septal defects detected by color Doppler imaging: evolution during fetal and first year of postnatal life. Ultrasound Obstet Gynecol 27(3): 266-273.

Baumgartner H, De Backer J, Babu-Narayan SV, Budts W, Chessa M, Diller GP, Lung B, Kluin J, Lang IM, Meijboom F, Moons P, Mulder BJM, Oechslin E, Roos-Hesselink JW, Schwerzmann M, Sondergaard L, Zeppenfeld K, Group ESCSD. 2020. 2020 ESC Guidelines for the management of adult congenital heart disease. European heart Journal.

Becker R, Schmitz L, Kilavuz S, Stumm M, Wegner RD, Bittner U. 2012. 'Normal' nuchal translucency: a justification to refrain from detailed scan? Analysis of 6858 cases with special reference to ethical aspects. Prenat Diagn 32(6): 550-556.

Becker R, Wegner RD. 2006. Detailed screening for fetal anomalies and cardiac defects at the 11-13-week scan. Ultrasound Obstet Gynecol 27(6): 613-618.

Benson DW. 2002. The genetics of congenital heart disease: a point in the revolution. Cardiol Clin 20(3): 385-394.

Berg C, Geipel A, Gembruch U. 2007. The four-chamber view in fetal echocardiography. Ultraschall Med 28(2): 132-151.

Berkhout WE. 2015. The ALARA-principle. Backgrounds and enforcement in dental practices. Ned Tijdschr Tandheelkd 122(5): 263-270.

Bernier PL, Stefanescu A, Samoukovic G, Tchervenkov CI. 2010. The challenge of congenital heart disease worldwide: epidemiologic and demographic facts. Semin Thorac Cardiovasc Surg Pediatr Card Surg Annu 13(1): 26-34.

Bertram DH, Weil, Sachweh. 2013. S2k Leitlinie Pädiatrische Kardiologie: Double Outlet Right Ventricle (DORV) im Kindes- und Jugendalter. AWMF Online.

Bhat AH, Kehl DW, Tacy TA, Moon-Grady AJ, Hornberger LK. 2013. Diagnosis of tetralogy of Fallot and its variants in the late first and early second trimester: details of initial assessment and comparison with later fetal diagnosis. Echocardiography 30(1): 81-87.

Botto LD, May K, Fernhoff PM, Correa A, Coleman K, Rasmussen SA, Merritt RK, O'leary LA, Wong LY, Elixson EM, Mahle WT, Campbell RM. 2003. A population-based study of the 22q11.2 deletion: phenotype, incidence, and contribution to major birth defects in the population. Pediatrics 112(1 Pt 1): 101-107.

Brite J, Laughon SK, Troendle J, Mills J. 2014. Maternal overweight and obesity and risk of congenital heart defects in offspring. international journal of obesity $38(6)$ : 878-882. 
Brooks PA, Khoo NS, Hornberger LK. 2014. Systolic and diastolic function of the fetal single left ventricle. J Am Soc Echocardiogr 27(9): 972-977.

Bull C. 1999. Current and potential impact of fetal diagnosis on prevalence and spectrum of serious congenital heart disease at term in the UK. British Paediatric Cardiac Association. Lancet 354(9186): 1242-1247.

Carvalho JS, Mavrides E, Shinebourne EA, Campbell S, Thilaganathan B. 2002. Improving the effectiveness of routine prenatal screening for major congenital heart defects. Heart 88(4): 387-391.

Cedergren MI, Kallen BA. 2003. Maternal obesity and infant heart defects. Obes Res 11(9): 1065-1071.

Centers for Disease C, Prevention. 1998. Trends in infant mortality attributable to birth defects--United States, 1980-1995. MMWR Morb Mortal Wkly Rep 47(37): 773-778.

Chaoui R, Heling K, Mielke G, Hofbeck M, Gembruch U. 2008. Quality standards of the DEGUM for performance of fetal echocardiography. Ultraschall Med 29(2): 197-200.

Chaoui R, Korner H, Bommer C, Goldner B, Bierlich A, Bollmann R. 1999. Prenatal diagnosis of heart defects and associated chromosomal aberrations. Ultraschall Med 20(5): 177-184.

Chau AC, Jones A, Sutherland M, Lilje C, Sernich S, Hagan J, Miller J. 2018. Characteristics of Isolated Ventricular Septal Defects Less Likely to Close In Utero. J Ultrasound Med 37(8): 1891-1898.

Clur SA, Bilardo CM. 2014. Early detection of fetal cardiac abnormalities: how effective is it and how should we manage these patients? Prenat Diagn 34(13): 1235-1245.

Clur SA, Ottenkamp J, Bilardo CM. 2009. The nuchal translucency and the fetal heart: a literature review. Prenat Diagn 29(8): 739-748.

Coding Committee of the Association for European Paediatric C. 2002. The European Paediatric Cardiac Code: the first revision. Cardiol Young 12 Suppl 2: 1-211.

Copel JA, Cullen M, Green JJ, Mahoney MJ, Hobbins JC, Kleinman CS. 1988. The frequency of aneuploidy in prenatally diagnosed congenital heart disease: an indication for fetal karyotyping. Am J Obstet Gynecol 158(2): 409-413.

Dashe JS, Mcintire DD, Twickler DM. 2009. Effect of maternal obesity on the ultrasound detection of anomalous fetuses. Obstet Gynecol 113(5): 1001-1007.

Dastgiri S, Stone DH, Le-Ha C, Gilmour WH. 2002. Prevalence and secular trend of congenital anomalies in Glasgow, UK. Arch Dis Child 86(4): 257-263. 
Daugirdaite V, Van Den Akker O, Purewal S. 2015. Posttraumatic stress and posttraumatic stress disorder after termination of pregnancy and reproductive loss: a systematic review. J Pregnancy 2015: 646345.

Davies V, Gledhill J, Mcfadyen A, Whitlow B, Economides D. 2005. Psychological outcome in women undergoing termination of pregnancy for ultrasound-detected fetal anomaly in the first and second trimesters: a pilot study. Ultrasound Obstet Gynecol 25(4): 389392.

Deutsche Gesellschaft Für Ultraschall in Der Medizin E.V. n.d. Mehrstufenkonzept und Zertifizierung. https://www.degum.de/sektionen/innere-medizin/mehrstufenkonzeptzertifizierung/stufe-ii-ausbilder.html.

Dgpk C. Jux, Vogel M, Scheewe J, Weil UJ. 2019. S2k Leitlinie Vorhofseptumdefekt. http://www.kinderkardiologie.org/fileadmin/user_upload/Leitlinien/2019_11_27_LL_ ASD.total_final.27.10.2019.li.pdf.

Dgpk M. Gorenflo, K.Schirmer, Dewald O. 2019. Leitlinie Der isolierte Ventrikelseptumdefekt. http://www.kinderkardiologie.org/fileadmin/user_upload/Leitlinien/2019_11_27_VSD nachFulda20.9.2019.final.li.pdf.

Dgpk: J. Weil, Bertram H, Sachweh JS. 2011. Leitlinie Pädiatrische Kardiologie: Fallot`sche Tetralogie. http://www.kinderkardiologie.org/fileadmin/user_upload/Leitlinien/20\%20LL\%20Fall otsche\%20Tetralogie.pdf.

Dgpk: A. Hager, Ovroutski S, Cesnjevar R. 2011. S2k Leitlinie Pädiatrische Kardiologie: Univentrikuläres Herz im Kindes- und Jugendalter. AWMF Online.

Dgpk: C. Rickers AH, T. Paul,. 2013. Leitlinie Pädiatrische Kardiologie: D-Transposition der großen Arterien. Retrieved

06.11.2020http://www.kinderkardiologie.org/fileadmin/user_upload/Leitlinien/25\%20 LL\%20D-Transposition\%20der\%20grossen\%20Arterien.pdf.

Dgpk: N. A. Haas, Jux C, Photiadis J, Kramer H-H. 2013. 29 Leitlinie Pädiatrische Kardiologie: Hypoplastisches Linksherzsyndrom (HLHS). http://www.kinderkardiologie.org/fileadmin/user_upload/Leitlinien/29\%20LL\%20Hy poplastisches\%20Linksherzsyndrom.pdf.

Dgpk: N. A. Haas, RüFfer A, Michel-Behnke I. 2020. S2k Leitlinie Truncus arteriosus communis (TAC). 
http://www.kinderkardiologie.org/fileadmin/user_upload/Leitlinien/LL_TAC_finalBer.pdf.

Dgpk: N. Haas, Rickers C, Feldmann RK-, Cesnjevar R. 2018. Leitlinie Pädiatrische Kardiologie: Aortenisthmusstenose.

http://www.kinderkardiologie.org/fileadmin/user_upload/Leitlinien/2018_06_20_LL_ _Aortenisthmusstenose.doc.pdf.

Dietrich K. 2006. Gynäkologie und Geburtshilfe. Heidelberg: Springer-Verlag, 533-534

Dittrich DS, Klaassen S, Kandolf R, Doenst T, Sieverding L. 2012. LL 28 Leitlinie

Pädiatrische Kardiologie: Primäre Kardiomyopathien.

http://www.kinderkardiologie.org/fileadmin/user_upload/Leitlinien/18\%20LL\%20Pri maere\%20Kardiomyopathien.pdf.

Dolk H, Loane M, Garne E, European Surveillance of Congenital Anomalies Working G. 2011. Congenital heart defects in Europe: prevalence and perinatal mortality, 2000 to 2005. Circulation 123(8): 841-849.

Ebrashy A, El Kateb A, Momtaz M, El Sheikhah A, Aboulghar MM, Ibrahim M, Saad M. 2010. 13-14-week fetal anatomy scan: a 5-year prospective study. Ultrasound Obstet Gynecol 35(3): 292-296.

Eydoux P, Choiset A, Le Porrier N, Thepot F, Szpiro-Tapia S, Alliet J, Ramond S, Viel JF, Gautier E, Morichon N, Et Al. 1989. Chromosomal prenatal diagnosis: study of 936 cases of intrauterine abnormalities after ultrasound assessment. Prenat Diagn 9(4): 255-269.

Fahed AC, Gelb BD, Seidman JG, Seidman CE. 2013. Genetics of congenital heart disease: the glass half empty. Circ Res 112(4): 707-720.

Ferencz C, Boughman JA. 1993. Congenital heart disease in adolescents and adults. Teratology, genetics, and recurrence risks. Cardiol Clin 11(4): 557-567.

Ferencz C, Neill CA, Boughman JA, Rubin JD, Brenner JI, Perry LW. 1989. Congenital cardiovascular malformations associated with chromosome abnormalities: an epidemiologic study. J Pediatr 114(1): 79-86.

Ferencz C, Rubin JD, Mccarter RJ, Brenner JI, Neill CA, Perry LW, Hepner SI, Downing JW. 1985. Congenital heart disease: prevalence at livebirth. The Baltimore-Washington Infant Study. Am J Epidemiol 121(1): 31-36.

Ficara A, Syngelaki A, Hammami A, Akolekar R, Nicolaides KH. 2020. Value of routine ultrasound examination at 35-37 weeks' gestation in diagnosis of fetal abnormalities. Ultrasound Obstet Gynecol 55(1): 75-80. 
Fiona A. Stennard RPH. 2005. T-box transcription factors and their roles in regulatory hierarchies in the developing heart. Development 132: 4897-4910.

Fmf Deutschland E.V. n.d. Arzt Info Zertifizierungsprozess FMF-Deutschland. Retrieved 31.10.2020https://www.fmf-deutschland.info/de/arzt-info/.

Forrester MB, Merz RD. 2004. Descriptive epidemiology of selected congenital heart defects, Hawaii, 1986-1999. Paediatr Perinat Epidemiol 18(6): 415-424.

Friedberg MK, Silverman NH, Moon-Grady AJ, Tong E, Nourse J, Sorenson B, Lee J, Hornberger LK. 2009. Prenatal detection of congenital heart disease. J Pediatr 155(1): 26-31, 31 e21.

Fung A, Manlhiot C, Naik S, Rosenberg H, Smythe J, Lougheed J, Mondal T, Chitayat D, Mccrindle BW, Mital S. 2013. Impact of prenatal risk factors on congenital heart disease in the current era. J Am Heart Assoc 2(3): e000064.

Geipel A, Gembruch U. 2012. Screening performance of first trimester nuchal translucency, ductus venosus blood flow and tricuspid regurgitation for cardiac defects. $\mathrm{Z}$ Geburtshilfe Neonatol 216(4): 157-161.

Gembruch U, Geipel A, Herberg U, Berg C. 2012. Fetal cardiac interventions. Z Geburtshilfe Neonatol 216(4): 162-172.

Gomez O, Martinez JM, Olivella A, Bennasar M, Crispi F, Masoller N, Bartrons J, Puerto B, Gratacos E. 2014. Isolated ventricular septal defects in the era of advanced fetal echocardiography: risk of chromosomal anomalies and spontaneous closure rate from diagnosis to age of 1 year. Ultrasound Obstet Gynecol 43(1): 65-71.

Götz D, KöHn A, Rißmann A, Spillner C, Vogt C. 2014. Fehlbildungsmonitoring SachsenAnhalt Jahresbericht 2014.

Haak Mc TJ, Van Vugt Jm. 2002. How successful is fetal echocardiographic examination in the first trimester of pregnancy? Ultrasound Obstet Gynecol Jul;20(1): 9-13.

Hansen M, Bower C, Milne E, De Klerk N, Kurinczuk JJ. 2005. Assisted reproductive technologies and the risk of birth defects--a systematic review. Hum Reprod 20(2): 328-338.

Harris JA, Francannet C, Pradat P, Robert E. 2003. The epidemiology of cardiovascular defects, part 2: a study based on data from three large registries of congenital malformations. Pediatr Cardiol 24(3): 222-235.

Hernandez-Andrade E, Patwardhan M, Cruz-Lemini M, Luewan S. 2017. Early Evaluation of the Fetal Heart. Fetal Diagn Ther 42(3): 161-173. 
Hill GD, Block JR, Tanem JB, Frommelt MA. 2015. Disparities in the prenatal detection of critical congenital heart disease. Prenat Diagn 35(9): 859-863.

Hoffman JI, Kaplan S. 2002. The incidence of congenital heart disease. J Am Coll Cardiol 39(12): 1890-1900.

Hoffman JIE, Kaplan S. 2002. The Incidence of Congenital Heart Disease. Journal of the American College of Cardiology Vol. 39, No. 12, 2002.

Holland BJ, Myers JA, Woods CR, Jr. 2015. Prenatal diagnosis of critical congenital heart disease reduces risk of death from cardiovascular compromise prior to planned neonatal cardiac surgery: a meta-analysis. Ultrasound Obstet Gynecol 45(6): 631-638.

Hollier LM, Leveno KJ, Kelly MA, Dd MC, Cunningham FG. 2000. Maternal age and malformations in singleton births. Obstet Gynecol 96(5 Pt 1): 701-706.

Holzer I, Husslein PW, Bettelheim D, Scheidl J, Kiss H, Farr A. 2019. Value of increased nuchal translucency in the era of noninvasive prenatal testing with cell-free DNA. Int J Gynaecol Obstet 145(3): 319-323.

Huggon IC, Defigueiredo DB, Allan LD. 2003. Tricuspid regurgitation in the diagnosis of chromosomal anomalies in the fetus at 11-14 weeks of gestation. Heart 89(9): 10711073 .

Hutchinson D, Mcbrien A, Howley L, Yamamoto Y, Sekar P, Motan T, Jain V, Savard W, Hornberger LK. 2017. First-Trimester Fetal Echocardiography: Identification of Cardiac Structures for Screening from 6 to 13 Weeks' Gestational Age. J Am Soc Echocardiogr 30(8): 763-772.

Hyett J, Moscoso G, Papapanagiotou G, Perdu M, Nicolaides KH. 1996. Abnormalities of the heart and great arteries in chromosomally normal fetuses with increased nuchal translucency thickness at 11-13 weeks of gestation. Ultrasound Obstet Gynecol 7(4): 245-250.

Iliescu D, Tudorache S, Comanescu A, Antsaklis P, Cotarcea S, Novac L, Cernea N, Antsaklis A. 2013. Improved detection rate of structural abnormalities in the first trimester using an extended examination protocol. Ultrasound Obstet Gynecol 42(3): 300-309.

Isuog: S. Eik-Nes. 2006. Cardiac screening examination of the fetus: guidelines for performing the 'basic' and 'extended basic' cardiac scan. Ultrasound Obstet Gynecol 27(1): 107-113. 
James WWPT, Bjorntorp P, Bray GA, Carroll KK, Chuchalin A, Dietz WH, Ehrlich GE, Hill JO. 2000. Obesity: preventing and managing the global epidemic. Report of a WHO consultation. World Health Organ Tech Rep Ser 894: i-xii, 1-253.

Jan Langman TWS. 1989. Taschenlehrbuch Embryologie, die normale menschliche Entwicklung und ihre Fehlbildungen. Stuttgart, New York: Georg Thieme Verlag, 536 Jenkins KJ, Correa A, Feinstein JA, Botto L, Britt AE, Daniels SR, Elixson M, Warnes CA, Webb CL, American Heart Association Council on Cardiovascular Disease in The Y. 2007. Noninherited risk factors and congenital cardiovascular defects: current knowledge: a scientific statement from the American Heart Association Council on Cardiovascular Disease in the Young: endorsed by the American Academy of Pediatrics. Circulation 115(23): 2995-3014.

Jicinska H, Vlasin P, Jicinsky M, Grochova I, Tomek V, Volaufova J, Skovranek J, Marek J. 2017. Does First-Trimester Screening Modify the Natural History of Congenital Heart Disease? Analysis of Outcome of Regional Cardiac Screening at 2 Different Time Periods. Circulation 135(11): 1045-1055.

Johnson CY, Honein MA, Hobbs CA, Rasmussen SA, National Birth Defects Prevention S. 2009. Prenatal diagnosis of orofacial clefts, National Birth Defects Prevention Study, 1998-2004. Prenat Diagn 29(9): 833-839.

Jortveit J, Oyen N, Leirgul E, Fomina T, Tell GS, Vollset SE, Eskedal L, Dohlen G, Birkeland S, Holmstrom H. 2016. Trends in Mortality of Congenital Heart Defects. Congenit Heart Dis 11(2): 160-168.

Kaisenberg CV, Chaoui R, HäUsler M, Kagan KO, Kozlowski P, Merz E, Rempen A, Steiner H, Tercanli S, Wisser J, Heling KS. 2016. Qualitätsanforderungen an die weiterführende differenzierte Ultraschalluntersuchung in der pränatalen Diagnostik (DEGUM-Stufen II und III) im Zeitraum 11-13+6 Schwanger- schaftswochen. Ultraschall in Med 37(03): 297-302.

Karadzov Orlic N, Egic A, Damnjanovic-Pazin B, Lukic R, Joksic I, Mikovic Z. 2019. Screening performance of congenital heart defects in first trimester using simple cardiac scan, nuchal translucency, abnormal ductus venosus blood flow and tricuspid regurgitation. Congenit Heart Dis 14(6): 1094-1101.

Khalil A, Nicolaides KH. 2013. Fetal heart defects: potential and pitfalls of first-trimester detection. Semin Fetal Neonatal Med 18(5): 251-260.

Knöpfle G. 1997. Die formale Entwicklung des Herzens beim Menschen. Der Gynäkologe 30(3): 170-180. 
Konorza TFM, J. Forkel. n.d. Angeborene Herzfehler. https://www.cardioguide.com/erkrankung/angeborene-herzfehler/\#Ursachen-für-angeborene-Herzfehler.

Kozlowski P, Burkhardt T, Gembruch U, Gonser M, Kahler C, Kagan KO, Von Kaisenberg C, Klaritsch P, Merz E, Steiner H, Tercanli S, Vetter K, Schramm T. 2019. DEGUM, OGUM, SGUM and FMF Germany Recommendations for the Implementation of First-Trimester Screening, Detailed Ultrasound, Cell-Free DNA Screening and Diagnostic Procedures. Ultraschall Med 40(2): 176-193.

Krapp M, Ludwig A, Axt-Fliedner R, Kreiselmaier P. 2011. First trimester fetal echocardiography: which planes and defects can be displayed during the daily routine in a prenatal medicine unit? Ultraschall Med 32(4): 362-366.

Ks Heling, Chaoui R. 2007. Der Dreigefäßblicks in der frühen fetalen Echokardiographie. Ein Hilfsmittel in der Diagnostik von Herzfehlern im I. Trimenon? - frühe fetale Echo. Zeitschrift für Geburtshilfe Neonatol 2007 211: S 2.

Lawson HW, Frye A, Atrash HK, Smith JC, Shulman HB, Ramick M. 1994. Abortion mortality, United States, 1972 through 1987. Am J Obstet Gynecol 171(5): 13651372.

Levy HL, Waisbren SE. 1983. Effects of untreated maternal phenylketonuria and hyperphenylalaninemia on the fetus. N Engl J Med 309(21): 1269-1274.

Lindinger A, Schwedler G, Hense HW. 2010. Prevalence of congenital heart defects in newborns in Germany: Results of the first registration year of the PAN Study (July 2006 to June 2007). Klin Padiatr 222(5): 321-326.

Liu Y, Chen S, Zuhlke L, Black GC, Choy MK, Li N, Keavney BD. 2019. Global birth prevalence of congenital heart defects 1970-2017: updated systematic review and meta-analysis of 260 studies. Int J Epidemiol 48(2): 455-463.

Maiz N, Valencia C, Emmanuel EE, Staboulidou I, Nicolaides KH. 2008. Screening for adverse pregnancy outcome by ductus venosus Doppler at 11-13+6 weeks of gestation. Obstet Gynecol 112(3): 598-605.

Makrydimas G, Sotiriadis A, Huggon IC, Simpson J, Sharland G, Carvalho JS, Daubeney PE, Ioannidis JP. 2005. Nuchal translucency and fetal cardiac defects: a pooled analysis of major fetal echocardiography centers. Am J Obstet Gynecol 192(1): 89-95.

Makrydimas G, Sotiriadis A, Ioannidis JP. 2003. Screening performance of first-trimester nuchal translucency for major cardiac defects: a meta-analysis. Am J Obstet Gynecol 189(5): 1330-1335. 
Marek J, Tomek V, Skovranek J, Povysilova V, Samanek M. 2011. Prenatal ultrasound screening of congenital heart disease in an unselected national population: a 21 -year experience. Heart 97(2): 124-130.

Martinez JM, Comas M, Borrell A, Bennasar M, Gomez O, Puerto B, Gratacos E. 2010. Abnormal first-trimester ductus venosus blood flow: a marker of cardiac defects in fetuses with normal karyotype and nuchal translucency. Ultrasound Obstet Gynecol 35(3): 267-272.

Mathews TJ, Macdorman MF. 2011. Infant mortality statistics from the 2007 period linked birth/infant death data set. Natl Vital Stat Rep 59(6): 1-30.

Mavrides E, Cobian-Sanchez F, Tekay A, Moscoso G, Campbell S, Thilaganathan B, Carvalho JS. 2001. Limitations of using first-trimester nuchal translucency measurement in routine screening for major congenital heart defects. Ultrasound Obstet Gynecol 17(2): 106-110.

Mcauliffe FM, Fong KW, Toi A, Chitayat D, Keating S, Johnson JA. 2005. Ultrasound detection of fetal anomalies in conjunction with first-trimester nuchal translucency screening: a feasibility study. Am J Obstet Gynecol 193(3 Pt 2): 1260-1265.

Merz E, Eichhorn KH, Von Kaisenberg C, Schramm T, Arbeitsgruppe Der D-S, Iii. 2012. [Updated quality requirements regarding secondary differentiated ultrasound examination in prenatal diagnostics (= DEGUM level II) in the period from $18+0$ to $21+6$ weeks of gestation]. Ultraschall Med 33(6): 593-596.

Messing B, Porat S, Imbar T, Valsky DV, Anteby EY, Yagel S. 2005. Mild tricuspid regurgitation: a benign fetal finding at various stages of pregnancy. Ultrasound Obstet Gynecol 26(6): 606-609; discussion 610.

Meyer-Wittkopf M, Hofbeck M. 2003. Two- and three-dimensional echocardiographic analysis of congenital heart disease in the fetus. Herz 28(3): 240-249.

Meyer-Wittkopf MK, Renate; Ziemer, Gerhard; Hofbeck, Michael; Walwiener, Diethelm,. 2003. In-utero-Diagnose und -Therapie angeborener Herzfehler: Grenzen und Möglichkeiten. Deutsches Ärzteblatt 100(50): A 3308-3313.

Miller A, Riehle-Colarusso T, Siffel C, Frias JL, Correa A. 2011. Maternal age and prevalence of isolated congenital heart defects in an urban area of the United States. Am J Med Genet A 155A(9): 2137-2145.

Minnella GP, Crupano FM, Syngelaki A, Zidere V, Akolekar R, Nicolaides KH. 2020. Diagnosis of major heart defects by routine first-trimester ultrasound examination: 
association with increased nuchal translucency, tricuspid regurgitation and abnormal flow in ductus venosus. Ultrasound Obstet Gynecol 55(5): 637-644.

Mitchell SC, Korones SB, Berendes HW. 1971. Congenital heart disease in 56,109 births. Incidence and natural history. Circulation 43(3): 323-332.

Moon-Grady A SS, Brook M, Rodriguez H, Hornberger Lk. 2012. Can a Complete Fetal Echocardiogram Be Performed at 12 to 16 Weeks' Gestation? Journal of the American Society of Echocardiography 25(12): 1342-1352.

Msd Manuals: Jeanne Marie Baffa M, Sidney Kimmel Medical College at Thomas Jefferson University, . 2018. 11/2018. Angeborene Herzfehler im Überblick. https://www.msdmanuals.com/de-de/profi/pädiatrie/angeboreneherzfehler/angeborene-herzfehler-im-überblick.

Neuman A, Huhta JC. 2006. First trimester screening for congenital heart disease. Minerva Cardioangiol 54(3): 337-354.

Nhlbi USA. 2014. How are congenital heart defects treated? https://www.nhlbi.nih.gov/health-topics/congenital-heart-defects.

Nicolaides KH. 2011. A model for a new pyramid of prenatal care based on the 11 to 13 weeks' assessment. Prenat Diagn 31(1): 3-6.

Nicolaides KH, Azar G, Byrne D, Mansur C, Marks K. 1992. Fetal nuchal translucency: ultrasound screening for chromosomal defects in first trimester of pregnancy. BMJ 304(6831): 867-869.

Pavlicek J, Gruszka T, Jaburek L. 2011. Risk factors in the medical history of pregnant women undergoing congenital heart defect prenatal screening. Ceska Gynekol 76(5): 386-392.

Persson M, Cnattingius S, Villamor E, Soderling J, Pasternak B, Stephansson O, Neovius M. 2017. Risk of major congenital malformations in relation to maternal overweight and obesity severity: cohort study of 1.2 million singletons. BMJ 357: 2563.

Persson M, Cnattingius S, Villamor E, Soderling J, Pasternak B, Stephansson O, Neovius M. 2017. Risk of major congenital malformations in relation to maternal overweight and obesity severity: cohort study of 1.2 million singletons. BMJ 357: j2563.

Pierpont ME, Basson CT, Benson DW, Jr., Gelb BD, Giglia TM, Goldmuntz E, Mcgee G, Sable CA, Srivastava D, Webb CL, American Heart Association Congenital Cardiac Defects Committee COCDITY. 2007. Genetic basis for congenital heart defects: current knowledge: a scientific statement from the American Heart Association Congenital Cardiac Defects Committee, Council on Cardiovascular Disease in the 
Young: endorsed by the American Academy of Pediatrics. Circulation 115(23): 30153038 .

Pinto NM, Keenan HT, Minich LL, Puchalski MD, Heywood M, Botto LD. 2012. Barriers to prenatal detection of congenital heart disease: a population-based study. Ultrasound Obstet Gynecol 40(4): 418-425.

Pius S, Abubakar Ibrahim H, Bello M, Bashir Tahir M. 2017. Complete Ectopia Cordis: A Case Report and Literature Review. Case Rep Pediatr 2017: 1858621.

Posada De La Paz M, Groft SC. 2010. Rare Diseases Epidemiology: Update and Overview. New York: Springer, 349-369

Pschyrembel Redaktion. 2019. Embryonale Herzentwicklung. https://www.pschyrembel.de/Embryonale\%20Herzentwicklung/A0VVK.

Quarello E, Lafouge A, Fries N, Salomon LJ, Cfef. 2017. Basic heart examination: feasibility study of first-trimester systematic simplified fetal echocardiography. Ultrasound Obstet Gynecol 49(2): 224-230.

Rasiah SV, Publicover M, Ewer AK, Khan KS, Kilby MD, Zamora J. 2006. A systematic review of the accuracy of first-trimester ultrasound examination for detecting major congenital heart disease. Ultrasound Obstet Gynecol 28(1): 110-116.

Reller MD, Strickland MJ, Riehle-Colarusso T, Mahle WT, Correa A. 2008. Prevalence of Congenital Heart Defects in Metropolitan Atlanta, 1998-2005. The Journal of Pediatrics 153(6): 807-813.

Rempen A. 2001. Standards in ultrasound examination in early pregnancy. Recommendation of DEGUM Stage III of the German Society of Ultrasound in Medicine (Gynecology and Obstetrics Section) and ARGUS (Working Group of Ultrasound Diagnosis of DGGG). December 2000 revision. Z Geburtshilfe Neonatol 205(4): 162-165.

Ren YY, Li XT, Gui YH, Yan YL, Chang C, Zhang JH, Sun L, Zhou YQ, Kong FB, Zhao W. 2008. Evaluation of the prenatal screening mode for fetal congenital heart diseases by ultrasound. Zhonghua Fu Chan Ke Za Zhi 43(8): 589-592.

Rickers DC, Horke A, Paul T. 2013. Leitlinie Pädiatrische Kardiologie: D-Transposition der großen Arterien.

http://www.kinderkardiologie.org/fileadmin/user_upload/Leitlinien/25\%20LL\%20DTransposition\%20der\%20grossen\%20Arterien.pdf.

Rki. 2014. Übergewicht und Adipositas. https://www.rki.de/DE/Content/Gesundheitsmonitoring/Themen/Uebergewicht_Adipo sitas/Uebergewicht_Adipositas_node.html. 
Rossi AC, Prefumo F. 2013. Accuracy of ultrasonography at 11-14 weeks of gestation for detection of fetal structural anomalies: a systematic review. Obstet Gynecol 122(6): 1160-1167.

Rowland TW, Hubbell JP, Jr., Nadas AS. 1973. Congenital heart disease in infants of diabetic mothers. J Pediatr 83(5): 815-820.

S. Dittrich, Schirrmeister J, Papan C, Weber LT. 2019. Repetitorium Kinder- und JugendmedizinSpringer-Verlag GmbH Deutschland, ein Teil von Springer Nature 2019, 281-293

Salvesen K, Lees C, Abramowicz J, Brezinka C, Ter Haar G, Marsal K, Board of International Society of Ultrasound In O, Gynecology. 2011. ISUOG statement on the safe use of Doppler in the 11 to $13+6$-week fetal ultrasound examination. Ultrasound Obstet Gynecol 37(6): 628.

Sankar MJ, Natarajan CK, Das RR, Agarwal R, Chandrasekaran A, Paul VK. 2016. When do newborns die? A systematic review of timing of overall and cause-specific neonatal deaths in developing countries. J Perinatol 36 Suppl 1: S1-S11.

Sharland G. 2004. Routine fetal cardiac screening: what are we doing and what should we do? Prenat Diagn 24(13): 1123-1129.

Sitzmann. 2002. Duale Reihe Pädiatrie. Stuttgart: Thieme Verlag, 329-330

Smrcek JM, Berg C, Geipel A, Fimmers R, Axt-Fliedner R, Diedrich K, Gembruch U. 2006. Detection rate of early fetal echocardiography and in utero development of congenital heart defects. J Ultrasound Med 25(2): 187-196.

Smrcek JM, Berg, C.,Geipel, A.,Fimmers, R., Diedrich, K., Gembruch, U. 2006. Early fetal echocardiography: heart biometry and visualization of cardiac structures between 10 and 15 weeks' gestation. J Ultrasound Med 25(2): 173-182; quiz 183-175.

Snijders RJ, Noble P, Sebire N, Souka A, Nicolaides KH. 1998. UK multicentre project on assessment of risk of trisomy 21 by maternal age and fetal nuchal-translucency thickness at 10-14 weeks of gestation. Fetal Medicine Foundation First Trimester Screening Group. Lancet 352(9125): 343-346.

Souka AP, Von Kaisenberg CS, Hyett JA, Sonek JD, Nicolaides KH. 2005. Increased nuchal translucency with normal karyotype. Am J Obstet Gynecol 192(4): 1005-1021.

Sun R, Liu M, Lu L, Zheng Y, Zhang P. 2015. Congenital Heart Disease: Causes, Diagnosis, Symptoms, and Treatments. Cell Biochem Biophys 72(3): 857-860. 
Syngelaki A, Chelemen T, Dagklis T, Allan L, Nicolaides KH. 2011. Challenges in the diagnosis of fetal non-chromosomal abnormalities at 11-13 weeks. Prenat Diagn 31(1): 90-102.

Talemal L, Donofrio MT. 2016. Hemodynamic consequences of a restrictive ductus arteriosus and foramen ovale in fetal transposition of the great arteries. J Neonatal Perinatal Med 9(3): 317-320.

Tararbit K, Lelong N, Thieulin AC, Houyel L, Bonnet D, Goffinet F, Khoshnood B, Group ES. 2013. The risk for four specific congenital heart defects associated with assisted reproductive techniques: a population-based evaluation. Hum Reprod 28(2): 367-374.

Torloni MR, Vedmedovska N, Merialdi M, Betran AP, Allen T, Gonzalez R, Platt LD, Group I-WFGS. 2009. Safety of ultrasonography in pregnancy: WHO systematic review of the literature and meta-analysis. Ultrasound Obstet Gynecol 33(5): 599-608.

Trines J, Hornberger LK. 2004. Evolution of heart disease in utero. Pediatr Cardiol 25(3): 287-298.

Tworetzky W, Mcelhinney DB, Reddy VM, Brook MM, Hanley FL, Silverman NH. 2001. Improved surgical outcome after fetal diagnosis of hypoplastic left heart syndrome. Circulation 103(9): 1269-1273.

U. Gembruch, Kempe A, Hellmund A, RöSing B, Willruth A, Berg C, Geipel A. 2013. Die Diagnostik fetaler Herzfehler im 1. und frühen 2. Trimenon - frühe fetale Echokardiografie. Geburtshilfe und Frauenheilkunde 73 (12).

Van Der Linde D, Konings EE, Slager MA, Witsenburg M, Helbing WA, Takkenberg JJ, Roos-Hesselink JW. 2011. Birth prevalence of congenital heart disease worldwide: a systematic review and meta-analysis. J Am Coll Cardiol 58(21): 2241-2247.

Van Velzen CL, Clur SA, Rijlaarsdam ME, Bax CJ, Pajkrt E, Heymans MW, Bekker MN, Hruda J, De Groot CJ, Blom NA, Haak MC. 2016. Prenatal detection of congenital heart disease--results of a national screening programme. BJOG 123(3): 400-407.

Voigt C, Schneider U, Schleußner E. 2018. Screeninguntersuchungen im ersten Trimenon in Zeiten von nicht- invasiven pränatalen Tests (NIPT). Ärzteblatt Thüringen 12/2018.

Volpe P, Ubaldo P, Volpe N, Campobasso G, De Robertis V, Tempesta A, Volpe G, Rembouskos G. 2011. Fetal cardiac evaluation at 11-14 weeks by experienced obstetricians in a low-risk population. Prenat Diagn 31(11): 1054-1061.

Von Kaisenberg C, Chaoui R, Hausler M, Kagan KO, Kozlowski P, Merz E, Rempen A, Steiner H, Tercanli S, Wisser J, Heling KS. 2016. Quality Requirements for the early 
Fetal Ultrasound Assessment at 11-13+6 Weeks of Gestation (DEGUM Levels II and III). Ultraschall Med 37(3): 297-302.

Warnes CA, Liberthson R, Danielson GK, Dore A, Harris L, Hoffman JI, Somerville J, Williams RG, Webb GD. 2001. Task force 1: the changing profile of congenital heart disease in adult life. J Am Coll Cardiol 37(5): 1170-1175.

Westin M, Saltvedt S, Bergman G, Kublickas M, Almstrom H, Grunewald C, Valentin L. 2006. Routine ultrasound examination at 12 or 18 gestational weeks for prenatal detection of major congenital heart malformations? A randomised controlled trial comprising 36,299 fetuses. BJOG 113(6): 675-682.

Whitworth M, Bricker L, Neilson JP, Dowswell T. 2010. Ultrasound for fetal assessment in early pregnancy. Cochrane Database Syst Rev(4): CD007058.

Wiechec M, Knafel A, Nocun A. 2015. Prenatal detection of congenital heart defects at the 11- to 13-week scan using a simple color Doppler protocol including the 4-chamber and 3-vessel and trachea views. J Ultrasound Med 34(4): 585-594.

World Health Organization. 04/2020. Body mass index - bmi. http://www.euro.who.int/en/health-topics/disease-prevention/nutrition/a-healthylifestyle/body-mass-index-bmi.

Wren C, Richmond S, Donaldson L. 2000. Temporal variability in birth prevalence of cardiovascular malformations. Heart 83(4): 414-419.

Yang Y, Zhang Y. 2014. [Ultrasound soft markers and fetal cardiac structural assessment at 11-14 weeks]. Zhonghua Fu Chan Ke Za Zhi 49(3): 188-192.

Yoon SA, Hong WH, Cho HJ. 2020. Congenital heart disease diagnosed with echocardiogram in newborns with asymptomatic cardiac murmurs: a systematic review. BMC Pediatr 20(1): 322 .

Zhu C, Liu Y-Q, Chen F-K, Hu D-L, Yu Z-B, Qian L-M. 2010. LYRM1, a Gene that Promotes Proliferation and Inhibits Apoptosis during Heart Development. molecules 15: 6974-6982.

Zidere V, Bellsham-Revell H, Persico N, Allan LD. 2013. Comparison of echocardiographic findings in fetuses at less than 15 weeks' gestation with later cardiac evaluation. Ultrasound Obstet Gynecol 42(6): 679-686. 


\section{Anhang}

\subsection{Aufklärung (Auszug) und Einverständniserklärung Ultraschalluntersuchung}

\section{Aufklärung vor Feindiagnostik (pränatale Diagnostik)}

Sehr geehrte Patientin,

Sie sind in unserer Praxis zur Durchführung einer speziellen geburtshilflichen Ultraschalluntersuchung. Mit dieser Untersuchung können wir Ihnen heute die normale Entwicklung des heranwachsenden Kindes im Mutterleib zeigen, eine Vielzahl von fetalen (kindlichen) Erkrankungen mit großer Sicherheit ausschließen und so auch helfen Zweifel und Ängste zu beseitigen.

Es können auch fetale Entwicklungsstörungen durch Versorgungsprobleme oder Organfehlentwicklungen entdeckt werden. Nach unserer Erfahrung profitieren die meisten dieser Kinder von der rechtzeitigen Diagnose - durch die frühzeitige Mitbetreuung durch spezialisierte Geburtshelfer und Kinderärzte und eine erfolgversprechende, spätere Therapie.

Bedenken Sie bitte auch, dass diese Ultraschalluntersuchung ein nicht eindeutiges oder gar ungünstiges Ergebnis erbringen kann und Sie dann möglicherweise sogar vor der Entscheidung für oder gegen eine invasive genetische Untersuchung des Ungeborenen (wie z.B. Fruchtwasserpunktion) stehen. Sie sollten sich vor dieser Untersuchung daher im Klaren sein, ob Sie dies überhaupt wollen.

Sie haben nach dem Gendiagnostikgesetz selbstverständlich auch das Recht auf Nichtwissen des Untersuchungsergebnisses oder Teilen davon und auch auf Vernichtung der gesamten Untersuchungsbefunde. Sollten Sie dies wünschen, notieren Sie Ihren Wunsch auf beigefügtem Blatt unter Bemerkungen und Fragen.

\section{Geplante Untersuchung im Rahmen der Feindiagnostik (19. - 22.SSW):}

\section{Ziel:}

Die weiterführende differentialdiagnostische Ultraschalldiagnostik (Feindiagnostik) beinhaltet den Ausschluss bzw. den Nachweis im Ultraschall erkennbarer Auffälligkeiten von Mutterkuchen, Fruchtwasser und der fetalen Anatomie, die auf eine fetale Erkrankung und / oder eine Entwicklungsstörung hinweisen können. 


\section{Indikationen:}

Die Indikationen für die Feindiagnostik sind in der Anlage 1c der Mutterschaftsrichtlinien festgelegt:

- Auffälligkeiten (z.B. unklare Befunde) im Rahmen der I. und II.

Mutterschaftsvorsorgeuntersuchung

- Differenzierung/ Prognoseeinschätzung fetaler Erkrankungen/ Entwicklungsstörungen

- Gezielter Ausschluss bzw. Nachweis einer fetalen Erkrankung bei Risikoschwangerschaft

- Psychische Belastung (z.B. Sorge der Patientin)

- Durchführung spezieller invasiver Eingriffe (wie Fruchtwasser- oder Mutterkuchenpunktion)

Darüber hinaus kann auch der Wunsch aus Sicherheitsgründen mehr über das heranwachsende Kind zu erfahren, Grund für die heutige Feindiagnostik sein (so genannte individuelle Gesundheitsleistung).

Die Indikation für die Ultraschalluntersuchung, die Klärung Ihrer Fragen und Sorgen oder Probleme aus Ihrer Gesundheits- und Familiengeschichte, Vorbefunde Ihres Frauenarztes/ Ihrer Frauenärztin werden vor und nach der Feindiagnostik mit Ihnen beraten.

Den Umfang dieser wichtigen Beratung, der natürlich auch abhängig von den Vorberatungen durch Ihren Frauenarzt/ Ihre Frauenärztin oder Humangenetiker ist, bestimmen Sie bitte unten im Abschnitt Bemerkungen und Fragen.

\section{Zeitpunkt:}

Optimaler Zeitpunkt für die Feindiagnostik ist der Bereich 19 bis 22. Schwangerschaftswoche und hängt sehr von den Sichtbedingungen (Bauchdeckenbeschaffenheit, Fruchtwassermenge, fetale Lage) und der Fragestellung ab. Bei einer Feindiagnostik zu einem früheren Zeitpunkt oder Hinweiszeichen auf Entwicklungsstörungen (z.B. Herzfehler) muss die Untersuchung oft später durch eine Verlaufskontrolle komplettiert werden.

\section{Inhalt:}

Die Untersuchung entspricht den Qualitätsanforderungen der DEGUM an die Feindiagnostik in der pränatalen Diagnostik im Zeitraum 18 - 22 Schwangerschaftswoche (aktualisierte Fassung in: Ultraschall in Med 2012; die Publikation liegt zu Ihrer Information in der Praxis aus) und wird auf höchstem technischen und personellen Niveau (DEGUM Stufe III) in unserer Praxis realisiert. 
Trotz sehr guter Gerätequalität, großer Sorgfalt und langjähriger Erfahrung der Untersucher muss ausdrücklich darauf aufmerksam gemacht werden, dass die Aussagefähigkeit der Ultraschalldiagnostik sehr von den Untersuchungsbedingungen abhängig ist und nicht alle Fehlbildungen, Veränderungen oder Erkrankungen grundsätzlich erkannt werden können.

Auch ist es möglich, dass kleinere Defekte nicht erkannt werden, z.B. ein Loch in der Herzscheidewand, eine Finger- oder Zehenfehlbildung sowie kleinere Defekte im Bereich der Wirbelsäule oder Lippenspalten.

Zudem entwickeln sich manche Erkrankungen und Entwicklungsstörungen erst im späteren Schwangerschaftsverlauf (z.B. einige Herzfehler, Knochen- oder Nierenerkrankungen, Gehirnfehlbildungen oder Folgen von Infektionen) und können daher zum heutigen Zeitpunkt noch nicht erkannt werden.

Die Untersuchungsbedingungen können dadurch erschwert sein, dass u.a. die Fruchtwassermenge gering ist, dass das Kind ungünstig liegt, dass die Bauchdecke der Schwangeren sehr kräftig ist oder Narben von Voroperationen die Qualität der Untersuchung mindern. Diese Faktoren können die Beurteilbarkeit des Feten einschränken und die Untersuchungszeit sehr verlängern.

Aufmerksam möchten wir außerdem darauf machen, dass Chromosomenstörungen wie z. B. eine Trisomie 21 (Down-Syndrom) oder Stoffwechselerkrankungen mit Ultraschall nicht erkannt oder ausgeschlossen werden können. Hierfür sind Eingriffe, wie Fruchtwasser- oder Mutterkuchenuntersuchungen oder fetale Blutuntersuchungen durch Nabelschnurpunktion nötig.

Bemerkungen und Fragen zur heutigen Ultraschalluntersuchung (Feindiagnostik):

Einverständniserklärung:

Ich habe die Informationen zur vorgeburtlichen Diagnostik gelesen. Ich hatte Gelegenheit, alle meine Fragen $\mathrm{zu}$ besprechen und habe die Erläuterungen verstanden. Ich benötige keine weitere Bedenkzeit. Mit der Durchführung der Untersuchung bin ich einverstanden und bestätige dies mit meiner Unterschrift.

$\square$ ja $\square$ nein

Entsprechend der Regelungen des Gendiagnostikgesetzes vom 01.02.2010 erkläre $\square$ ja $\square$ nein 
ich:

Ich bin damit einverstanden, dass die erhobenen Ergebnisse in Papierform sowie in elektronischer Form, entsprechend der gesetzlichen Vorgaben gespeichert und in pseudonymisierter Form für wissenschaftliche Zwecke oder für Zwecke der Qualitätssicherung genutzt/publiziert werden.

Ich wünsche die Mitteilung der Untersuchungsergebnisse an meine behandelnden Ärzte / Ärztinnen:

Erfurt, den 


\subsection{Patientenanschreiben, Einverständniserklärung und Fragebogen}

\section{Patienteninformation}

Sehr geehrte Damen und Herren, die Praxis für Pränatalmedizin Dr. Kähler, Erfurt und das Universitätsklinikum Jena, Abteilung Geburtshilfe, arbeiten zurzeit an einer Verlaufsstudie mit Patienten, die zur pränatalen Untersuchung in Erfurt vorstellig waren.

Ziel der Arbeit ist es, die pränatale (vor Geburt) Untersuchung des Kindes mit dem postnatalen (nach Geburt) Zustand zu vergleichen. Schwerpunkte dabei sind die Einstellung des Herzens und der Nabelschnur. Im Verlauf der Studie wird es bei einigen Patienten nötig sein, Daten bezüglich der Nabelschnur aus der Geburtsklinik anzufordern. Hierfür benötigen wir Ihr Einverständnis.

Wir wären Ihnen sehr verbunden, wenn Sie sich kurz Zeit nehmen würden, um die folgenden Fragen zu beantworten und die Einverständniserklärung zu unterschreiben.

Wir möchten Sie bitten, die Antworten aus dem Mutterpass (S.15) oder dem gelben U-Heft zu entnehmen.

Alternativ können Sie sich auch gern telefonisch melden und die Antworten mündlich durchgeben oder auch per E-Mail senden an:

Auch für weitere Fragen bezüglich der Studie stehen wir Ihnen jederzeit zur Verfügung.

Bitte senden Sie die Einverständniserklärung und den Fragebogen zurück. Ein frankierter Rückumschlag liegt bei. 


\section{Einverständniserklärung zur Teilnahme an den Studien}

1. „Bedeutung der Darstellung der plazentaren Nabelschnurinsertion durch Zwei- und Drei-D Sonografie im ersten und zweiten Trimenon“

2. „Erkennbarkeit angeborener Herzfehler durch eine standardisierte Untersuchung des fetalen Herzens im ersten Trimenon “

sowie zur Erhebung von Daten im Rahmen dieser Studie

Name, Vorname:

Ich habe alle Punkte der Patienteninformation gelesen und verstanden. Alle mich interessierenden Fragen konnte ich entweder einer Ärztin oder der Studienbetreuerin stellen und wurden in verständlicher Weise beantwortet.

Hiermit erkläre ich mich einverstanden, zur Teilnahme an den oben genannten Studien und zur Erhebung der für die Studien relevanten Daten in personenbezogener Form bei meinem behandelnden Arzt /Ärztin, sowie zur anonymisierten Weiterverarbeitung.

Mir ist bekannt, dass meine Teilnahme freiwillig ist und ich jederzeit ohne Angabe von Gründen widerrufen kann.

Ort, Datum

Unterschrift 
Fragebogen (Auszug der für diese Studie relevanten Fragen)

\section{Angaben der Kindsmutter:}

1. Name, Vorname:

2. Sind Ihnen Herzfehler in Ihrer Familie bekannt?

$$
\begin{aligned}
& \square \text { nein } \\
& \square \text { ja }
\end{aligned}
$$

falls ja, bitte genauen Verwandtschaftsgrad im Bezug zum Kind angeben und welche Art von Herzfehler

\section{Angaben zum Kind:}

1. Geburtsdatum:

2. Schwangerschaftswoche der Geburt (Im Pass S. 15 abgekürzt als SSW)

3. Gibt es bei dem Kind Auffälligkeiten bezüglich des Herzens?

4. Wie wurde das Kind geboren?
$\square$ Kaiserschnitt:
Normaler Geburtsweg

Falls Kaiserschnitt, bitte

Grund dafür angeben

5. War das Kind nach der Geburt auf der Intensivstation

$$
\begin{aligned}
& \square \text { nein } \\
& \square \text { ja }
\end{aligned}
$$

falls ja, warum

6. gab es sonst Auffälligkeiten bei dem Kind?
$\square$ nein
$\square$ ja

falls ja, welche? 


\subsection{Ausführung statistische Erhebung}

1. 4-Feldertafel: Vergleich von allen Fällen mit Herzfehlern (Gegenereignis kein Herzfehler) mit auffälligen kardialen Ersttrimestereinstellungen (Gegenereignis unauffällige kardiale Ersttrimestereinstellungen)

\begin{tabular}{|l|l|l|l|l|}
\hline & & Herzfehler & & Summe \\
\hline & & Ja & nein & \\
\hline Testergebnis & Positiv & 20 & 48 & 68 \\
\hline & negativ & 31 & 1084 & 1115 \\
\hline Summe & & 51 & 1132 & 1183 \\
\hline
\end{tabular}

Sensitivität: 39,21 \%; Spezifität: 95,75 \%; PPW: 29,41 \%; NPW: 97,21\%

2. 4-Feldertafel: Vergleich von Fällen mit moderaten oder schweren Herzfehlern (Gegenereignis Fälle ohne Herzfehler - milde Herzfehler fallen bei dieser Betrachtung raus) mit auffälligen (pathologisch/kontrollbedürftig/nicht beurteilbar) kardialen Ersttrimestereinstellungen (Gegenereignis unauffällige kardiale Einstellungen).

\begin{tabular}{|l|l|l|l|l|}
\hline & & Herzfehler & & Summe \\
\hline & & Ja & nein & \\
\hline Testergebnis & Positiv & 19 & 48 & 67 \\
\hline & negativ & 2 & 1084 & 1086 \\
\hline Summe & & 21 & 1132 & 1153 \\
\hline
\end{tabular}

Sensitivität: 90,47 \%; Spezifität: 95, 75 \%; PPW: 28,35 \%; NPW: 99,81 \%

3. 4-Feldertafel: Vergleich von Fällen mit moderaten oder schweren Herzfehlern (Gegenereignis kein Herzfehler - milde Herzfehler fallen raus) mit Fällen mit pathologischem oder kontrollbedürftigem kardialen Einstellungen im Ersttrimesterscreening (Gegenereignis unauffällige/nicht beurteilbare kardiale Einstellungen). 


\begin{tabular}{|l|l|l|l|l|}
\hline & & $\begin{array}{l}\text { Herzfehler } \\
\text { schwer/moderat }\end{array}$ & $\begin{array}{l}\text { Kein } \\
\text { Herzfehler }\end{array}$ & Summe \\
\hline & & Ja nein & \\
\hline $\begin{array}{l}\text { Testergebnis pathologisch/ } \\
\text { kontrollbedürftig }\end{array}$ & Positiv & 17 & 0 & 17 \\
\hline & negativ & 4 & 1132 & 1136 \\
\hline Summe & & 21 & 1132 & 1153 \\
\hline
\end{tabular}

Sensitivität: 80,95 \%; Spezifität: $100 \%$; PPW: $100 \%$; NPW: 99,6 \%

4. 4-Feldertafel: Vergleich von Fällen mit moderaten oder schweren Herzfehlern (Gegenereignis kein Herzfehler) mit Fällen mit pathologischem oder kontrollbedürftigem kardialen Einstellungen im Ersttrimesterscreening (Gegenereignis unauffällige kardiale Einstellungen - Nicht beurteilbare Fälle fallen raus).

\begin{tabular}{|l|l|l|l|l|}
\hline & & $\begin{array}{l}\text { Herzfehler } \\
\text { schwer/moderat }\end{array}$ & $\begin{array}{l}\text { Kein/milder } \\
\text { Herzfehler }\end{array}$ & Summe \\
\hline $\begin{array}{l}\text { Testergebnis } \\
\text { pathologisch/ } \\
\text { Kontrollbedürftig }\end{array}$ & positiv & 17 & 0 & 17 \\
\hline $\begin{array}{l}\text { Testergebnis } \\
\text { unauff/nicht } \\
\text { beurteil bar }\end{array}$ & negativ & 4 & 1162 & 1166 \\
\hline Summe & & 21 & 1162 & 1183 \\
\hline
\end{tabular}

Sensitivität: 80,95 \%; Spezifität: $100 \%$; PPW: $100 \%$; NPW: 99,65 \% 


\subsection{Abbildungsverzeichnis}

Abb. 1: Embryonale Entwicklung des Herzens

Abb. 2: physiologische Blutzirkulation bei einem Fötus

Abb. 3: $\quad$ Double inlet ventricle

Abb. 4: $\quad$ HLHS: mit hypoplastischem, hypokinetischem LV, dysplastischer Mitralklappe, Aortenklappenatresie und hypoplastischem Aortenbogen

Abb. 5: $\quad$ Beispielvariante DORV bei dem die Aorta rechts vor der Pulmonalarterie liegt Abb. 6: Beispiel TGA mit Atrioventrikulärer Diskonkordanz, RA verbunden durch Mitralklappe zu LV, LA verbunden durch Trikuspidalklappe zu RV, Aorta enspringt RV, Pulmonalarterie entspringt LV, Parallelverlaufende große Gefäße, Ao liegt vor PA

Abb. 7: Fallot-Tetralogie mit subaortalem malalignment-VSD, Reitender Aorta, Pulmonalstenose und rechtsventrikulärer Hypertrophie

Abb. 8: $\quad$ Aortenisthmusstenose mit Diskrepanz zwischen den Ventrikeln (verengerter linker Ventrikel)

Abb. 9: $\quad$ ASD Typ I

Abb. 10: Ventrikelseptumdefekt

Abb. 11: Beispiel TAC: Aorta und der Stamm der Pulmonalarterie entspringen aus einem gemeinsamen Gefäßstamm

Abb. 12: Sonografische Standardeinstellungen der fetalen Anatomie- und optionale Parameter der FMF

Abb. 13: Normalbefund 4KB mit Farbdoppler

Abb. 14: Normalbefund LVOT mit Farbdoppler

Abb. 15: $\quad$ Normalbefund RVOT mit Farbdoppler

Abb. 16: $\quad$ zu überprüfende sonoanatomische Parameter im Zeitraum von $18+0$ bis $21+6$ SSW im Rahmen der weiterführenden differentialdiagnostischen Ultraschalluntersuchung

Abb. 17: Darstellung der 2 klassifizierten Herzfehler

Abb. 18: Aufteilung aller pränatal gefundenen Herzfehler (CHD) nach isoliert oder nicht isoliert in die schwere der vorliegenden Herzfehlerform

Abb. 19: Darstellung der kardialen Einstellung in der frühen FD bei Feten mit schweren oder moderaten Herzfehlern 
Abb. 20: Darstellung der kardialen Einstellung in der frühen FD bei Feten mit leichten Herzfehlern

Abb. 21: Aufteilung der Fälle in denen die kardialen Einstellungen in der frühen FD als pathologisch oder kontrollbedürftig beschrieben wurden nach klassifizierten Herzfehlern

Abb. 22: $\quad$ Beurteilung 4-Kammerblick in der frühen fetalen Herzuntersuchung

Abb. 23: $\quad$ Beurteilung LVOT und RVOT in der frühen fetalen Herzuntersuchung

Abb. 24: Darstellung Nackentransparenz nach Quartilen, eingeteilt in Feten ohne Herzfehler und Feten mit schwerem oder moderatem Herzfehler sowie leichte Herzfehler

Abb. 25: $\quad$ Fall 1: univentrikuläres Herz

Abb. 26: $\quad$ Fall 4: univentrikuläres Herz

Abb. 27: $\quad$ Fall 8: univentrikuläres Herz

Abb. 28: $\quad$ Fall 9: d-TGA

Abb. 29: Fall 10: Vitium cordis: re Ventrikel geringer perfundiert, retrograde Perfusion des DAB

Abb. 30: $\quad$ Fall 14: RVOT mit retrograder Aortenperfusion

Abb. 31: Fall 16: univentrikuläres Herz

Abb. 32: $\quad$ Fall 18: AVSD

Abb. 33: $\quad$ Fall 21: VSD mit bidirektionalem Shunt

Abb. 34: $\quad$ Fall 1: 3 Gefäßblick: rechtsverlaufender Aortenbogen

Abb. 35: Vergleich der kardialen Diagnosen in der FFH (frühen fetalen Herzuntersuchung) und Echokardiographie ab 18. SSW bei Feten mit schwerem oder moderatem Herzfehler

Abb. 36: Vergleich der kardialen Diagnosen in der FFH (frühen fetalen Herzuntersuchung) und in der Echokardiographie ab der 18. SSW bei allen Fällen in denen entweder Ersttrimesterscreening als auffällig (,pathologisch/kontrollbedürftig/nicht beurteilbar"), oder die spätere Echokardiographie als auffällig eingeschätzt wurde 


\subsection{Tabellenverzeichnis}

Tab. 1: $\quad$ CHD assoziierte Fehlbildungen

Tab. 2: $\quad$ Typische mit Herzfehlern assoziierte genetische Störungen

Tab. 3: Nomenklatur der Screening-Untersuchungen im 1. Trimenon

Tab. 4: $\quad$ Ergebnisse genetischer Untersuchung

Tab. 5: $\quad$ Screening Performance der kardialen Einstellungen

Tab. 6: Anteil Untersuchungsmethode im Gesamtkollektiv, bei eingeschränkten USB und nicht beurteilbaren kardialen Einstellungen

Tab. 7: statistische Auswertung weiterer Risikofaktoren für angeborene Herzfehler

Tab. 8: Fälle mit schweren oder moderaten Herzfehlern

Tab. 9: $\quad$ Fall mit mildem Herzfehler

Tab. 10: postnatal nicht bestätigte milde Herzfehler

Tab. 11: postnatal erstdiagnostizierter Herzfehler 


\subsection{Danksagung}

Ich danke dem Team der Praxis für Pränatalmedizin: Franziska, Manuela, Nadine und Susanne die mich über die Jahre unterstützt haben. Danke für die freundliche und gute Atmosphäre, in der ich sehr gern gearbeitet habe.

Mein Dank gilt außerdem Herrn Professor Schleußner für die Betreuung und schnelle Zuarbeit.

Ebenso bedanke ich mich bei Frau Dipl.-Math. Oec. Wedekind für die Beratung bei statistischen Fragestellungen.

Ganz besonderer Dank richtet sich an meine Mutter Christiane Kähler für die ständige Erreichbarkeit. Den Spagat zwischen Korrekturen und Enkelbetreuung, hätte ich mir nicht besser wünschen können. Durch ihre positive Bestärkung und der Glaube an meine Fähigkeiten ist eine Motivation entstanden, die mich durch den gesamten Prozess der Arbeit getragen hat.

Zudem möchte ich mich bei meinem Mann Jakob und meinem Sohn Leander bedanken, die auf gemeinsame Familienzeit verzichtet haben. Erwähnen möchte ich auch die Geduld meiner Tochter Alma, die mich in meinem Schreibprozess als noch ungeborenes Kind begleitet und viele Stunden in der Bibliothek ausgehalten hat.

Ein Dank möchte ich ebenso an meine Freundin Paula richten, die meine Laune durch ihre Leichtigkeit im Leben immer hochgehalten hat.

Vielen Dank auch Julia für die schnelle und professionelle Korrektur. 
Anhang

10.7 Lebenslauf 


\subsection{Ehrenwörtliche Erklärung}

Hiermit erkläre ich, dass

mir die Promotionsordnung der Medizinischen Fakultät der Friedrich- Schiller-Universität bekannt ist,

ich die Dissertation selbst angefertigt habe und alle von mir benutzen Hilfsmittel, persönliche Mitteilungen und Quellen in meiner Arbeit angegeben sind,

mich folgende Personen bei der Auswertung des Materials sowie bei der Herstellung des Manuskripts unterstützt haben: Priv. Doz. Dr. med. habil. C. Kähler und Prof. Dr. med. E. Schleußner und Frau Dipl.-Math. Oec. Wedekind,

die Hilfe eines Promotionsberaters nicht in Anspruch genommen wurde und dass Dritte weder unmittelbar noch mittelbar geldwerte Leistungen von mir für Arbeiten erhalten haben, die im Zusammenhang mit dem Inhalt der vorgelegten Dissertation stehen,

dass ich die Dissertation noch nicht als Prüfungsarbeit für eine staatliche oder andere wissenschaftliche Prüfung eingereicht habe und

dass ich die gleiche, eine in wesentlichen Teilen ähnliche oder eine andere Abhandlung nicht bei einer anderen Hochschule als Dissertation eingereicht habe.

Leipzig, den 